\title{
Inter-Individual Differences as Instrument to Investigate the Mechanisms in Metacontrast Masking
}

\author{
DisSERTATION
}

zur Erlangung des mathematisch-naturwissenschaftlichen

Doktorgrades

„Doctor rerum naturalium“ (Dr. rer. nat.)

der Georg-August-Universität Göttingen

$$
\text { im Promotionsprogramm Biologie }
$$

der Georg-August University School of Science (GAUSS)

\author{
vorgelegt von \\ Mareen Berndt \\ aus Görlitz
}

Göttingen, 2016 



\title{
Inter-Individual Differences as Instrument to Investigate the Mechanisms in Metacontrast Masking
}

\section{DisSERTATION}

\author{
for the award of the degree \\ „Doctor rerum naturalium“ (Dr. rer. nat.) \\ of the Georg-August-University of Göttingen
}

\begin{abstract}
within the doctoral program Biology
of the Georg-August University School of Science (GAUSS)

submitted by

Mareen Berndt

from Görlitz
\end{abstract}

Göttingen, 2016 

Betreuungsausschuss

Prof. Dr. Uwe Mattler

Abteilung für Experimentelle Psychologie, Georg-Elias-Müller-Institut für Psychologie, GeorgAugust-Universität Göttingen

Prof. Dr. Roland Grabner

Arbeitsbereich Begabungsforschung, Institut für Psychologie, Karl-Franzens-Universität Graz

Dr. Thorsten Albrecht

Abteilung für Experimentelle Psychologie, Georg-Elias-Müller-Institut für Psychologie, GeorgAugust-Universität Göttingen

Mitglieder der Prüfungskommission

Referent: Prof. Dr. Uwe Mattler

Abteilung für Experimentelle Psychologie, Georg-Elias-Müller-Institut für Psychologie, GeorgAugust-Universität Göttingen

Koreferent: Prof. Dr. Roland Grabner

Arbeitsbereich Begabungsforschung, Institut für Psychologie, Karl-Franzens-Universität Graz

Weitere Mitglieder der Prüfungskommission:

apl. Prof. Dr. Uta Lass

Abteilung für Experimentelle Psychologie, Georg-Elias-Müller-Institut für Psychologie, GeorgAugust-Universität Göttingen, (i.R. April 2016)

Prof. Dr. Thomas Schmidt

Fachgebiet Allgemeine Psychologie, Fachbereich Sozialwissenschaften, Technische Universität Kaiserslautern

Prof. Dr. Annekathrin Schacht

Courant Forschungszentrum „Textstrukturen“, Nachwuchsgruppe Experimentelle Psycholinguistik, Georg-Elias-Müller-Institut für Psychologie, Georg-August-Universität Göttingen

apl. Prof. Dr. York Hagmayer

Abteilung für Kognitionswissenschaft und Entscheidungspsychologie, Georg-Elias-Müller-Institut für Psychologie, Georg-August-Universität Göttingen

Tag der mündlichen Prüfung: 05.09.2016 



\section{Acknowledgment}

I would like to thank to thank my research assistants Sarah Kröber, Birte Clausen, and Verena Klar for their countless hours of data acquisition, you made a wonderful job and without you the accomplishment of the experiments would not have been possible, my supervisor Thorsten Albrecht for his methodological and technical support and his extensive knowledge and farsightedness about this project, you taught me a lot and contributed essentially to the success of this work, my examiners Uwe Mattler and Roland Grabner for their feedback and inspiring suggestions to this work, Uta Lass for her wise and well-meaning advices, my colleagues Martina Wernicke, Anne Sommerfeld, Nicolas Becker, Nora Koster, Christian Valuch, and Maximilian Stein for their exchange of knowledge and their sympathetic ears, you made everyday work even more pleasant, and last but not least Ingo Geestmann, my mental haven and inexhaustible source of encouragement, I appreciate you a lot. 



\section{Abstract}

In metacontrast masking the visibility of the first stimulus (the target) is reduced due to the appearance of the second stimulus (the mask). Two masking functions mainly occur: "Type A", when visibility is increasing with increasing SOA, and "Type B", where visibility is high in short and long SOAs and dropped to a minimum in between. In five studies it systematically investigated which influence different experimental parameters have on metacontrast masking functions. The longer the mask duration becomes in relation to the target duration, the more the minimum of the masking function shifts towards shorter SOAs, and the more Type-A-like (monotonically increasing) the function becomes. In the center and in the periphery masking is stronger in small stimuli than in large stimuli, and the SOA of the strongest masking occurred at the shortest SOA in both stimulus sizes. Masking functions are identical for predictable and unpredictable stimulus presentation locations. Apparent motion is better detected in long SOAs than in short SOAs.

Furthermore, inter-individual differences were found, which were used to get insights in the mechanisms involved in metacontrast masking. Some participants show a Type A masking function and mostly report to perceive apparent movements in the sequence of both stimuli, mainly in long SOAs. Others show a Type B masking function and mostly report to perceive negative afterimages in the shape of the target inside the mask, mainly in short SOAs. Type A and Type B observers do differ in their top-down processing, but not in their bottom-up processing of metacontrast stimuli. Two processes could be revealed, which may be involved. According to the Integration-Segregation-Theory the perception of two stimuli being segregated, enabling apparent moving images, are associated to the segregation process. The percept of simultaneous, conjoint image of two physically succeeding stimuli are associated to the integration process. As Process 1 is strongly associated with a Type A masking function, which in turn goes along with a percept of motion, it may correspond to the segregation process. As Process 2 is strongly associated with a Type B masking function, which in turn goes along with a percept of negative afterimages inside of the mask stimulus, it may correspond to the integration process. It is assumed that these two processes are involved in the conscious perception of the target stimulus in metacontrast masking. 



\section{Zusammenfassung}

In der Metakontrastmaskierung wird die Sichtbarkeit des ersten Stimulus (Target) durch das Auftreten eines zweiten Stimulus (Maske) reduziert. Zwei Maskierungsfunktionen (MF) treten hauptsächlich auf: Typ A, wenn die Sichtbarkeit mit ansteigender SOA zumimmt, und Typ-B, wenn die Sichtbarkeit in kurzer und langer SOA hoch ist und auf ein Minimum in mittlerer SOA abfällt. In fünf Studien wurde systematisch untersucht welchen Einfluss experimentelle Parameter auf das Auftreten der MF haben. Je länger die Maske im Verhältnis zum Target präsentiert wird, desto weiter verschiebt sich das Minimum der MF hin zu kürzerer SOA und desto mehr ähnelt sie einer Typ-A-MF (monoton ansteigend). Die Maskierung ist in kleinen Stimuli stärker als in großen Stimuli, sowohl im Zentrum als auch in der Peripherie. Bei beiden Stimulusgrößen findet bei der kürzesten SOA die stärkste Maskierung statt. MF unterscheiden sich nicht, wenn sich die Vorhersagbarkeit der Präsentationsorte der Stimuli unterscheidet. Scheinbewegungen werden in langen SOAs eher wahrgenommen als in kurzen SOAs.

Darüber hinaus wurden inter-individuelle Unterschiede gefunden, die Einblicke in die Mechanismen erlauben, die in der Metakontrastmaskierung beteiligt sind. Einige Versuchspersonen zeigen eine Typ-A-MF und berichten Scheinbewegungen in der Abfolge der Stimuli wahrzunehmen, hauptsächlich in langen SOAs. Andere zeigen eine Typ-B-MF und berichten negative Nachbilder in Form des Tagets im Inneren der Maske wahrzunehmen, hauptsächlich in kurzen SOAs. Typ-A- und Typ-B-Versuchspersonen unterscheiden sich in ihrer Top-DownVerarbeitung der Stimuli, nicht aber in ihrer Bottom-Up-Verarbeitung. Zwei Prozesse stellten sich heraus, die in der Verarbeitung der Metakontraststimuli beteiligt sein könnten. Nach der Integrations-Segregations-Theorie steht die getrennte (segregierte) Wahrnehmung zweier Stimuli, die Scheinbewegungen ermöglicht, mit dem Segregationsprozess in Zusammenhang. Dagegen steht die gleichzeitige (integrierte) Wahrnehmung zweier nacheinander folgender Stimuli mit dem Integrationsprozess in Zusammenhang. Da Prozess 1 stark mit einer Typ-A-MF verknüpft ist, einhergehend mit der Wahrnehmung von Scheinbewegungen, könnte dieser Prozess dem Segregationsprozess entsprechen. Da Prozess 2 stark mit einer Typ-B-MF verknüpft ist, einhergehend mit der Wahrnehmung negativer Nachbilder, könnte dieser Prozess dem Integrationsprozess entsprechen. Es wird angenommen, dass diese beiden Prozesse an der bewussten Wahrnehmung des Targets in der Metakontrastmaskierung beteiligt sind. 



\section{Contents}

1 General Introduction 1

1.1 Metacontrast Masking . . . . . . . . . . . . . . . . . 1

1.2 Processing Models and Theories . . . . . . . . . . . . . . 3

1.3 Inter-Individual Differences $\ldots \ldots \ldots \ldots \ldots$

1.4 Aim and Outline of Studies . . . . . . . . . . . . . . . . . 7

2 General Methods 9

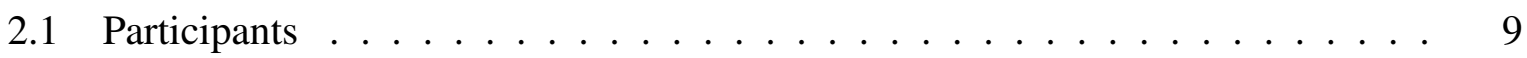

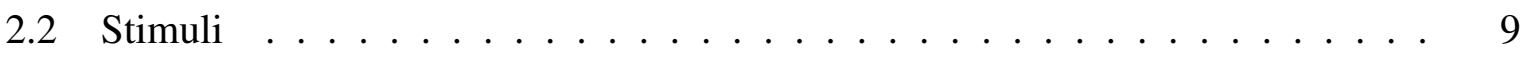

2.3 Experimental Set-Up . . . . . . . . . . . . . . . . . . 9

2.4 Data Analysis . . . . . . . . . . . . . . . . . . . 11

2.4.1 Signal Detection Theory . . . . . . . . . . . . . . . 11

2.4 .2 Factor Analysis . . . . . . . . . . . . . . . . . 11

3 Study 1 - Effect of the Ratio of Target Duration to Mask Duration 13

3.1 Methods ............................. 15

3.1 .1 Participants .......................... 15

3.1 .2 Experimental Set-Up . . . . . . . . . . . . . . . 15

3.1 .3 Data Analysis . . . . . . . . . . . . . . . . 16

3.2 Results . . . . . . . . . . . . . . . . . . 16

3.2 .1 Experiment $1.1 \ldots \ldots \ldots \ldots \ldots$

3.2 .2 Experiment $1.2 \ldots \ldots \ldots \ldots \ldots$

3.2 .3 Experiment $1.3 \ldots \ldots \ldots \ldots \ldots$

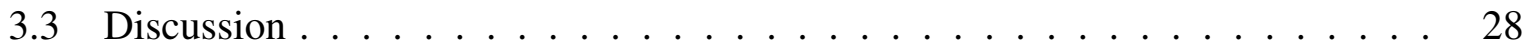

3.3 .1 Experiment $1.1 \ldots \ldots \ldots \ldots \ldots \ldots \ldots$

3.3 .2 Experiment $1.2 \ldots \ldots \ldots \ldots \ldots$

3.3 .3 Experiment $1.3 \ldots \ldots \ldots \ldots \ldots \ldots \ldots$

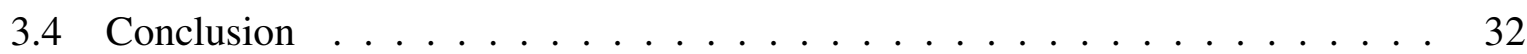




\section{Contents}

4 Study 2 - Effect of Stimulus Size and Eccentricity 35

4.1 Methods . . . . . . . . . . . . . . . . . . . 37

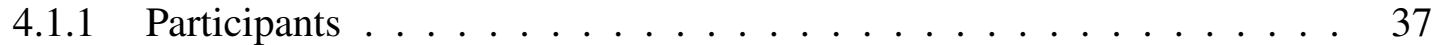

4.1 .2 Experimental Set-Up . . . . . . . . . . . . . . 37

4.1 .3 Data Analysis . . . . . . . . . . . . . . . . 38

4.2 Results . . . . . . . . . . . . . . . . . . . 38

$4.2 .1 \quad$ Experiment $2.1 \ldots \ldots \ldots \ldots \ldots$

4.2 .2 Experiment $2.2 \ldots \ldots \ldots \ldots \ldots$

4.2 .3 Experiment $2.3 \ldots \ldots \ldots \ldots \ldots \ldots$

4.3 Discussion . . . . . . . . . . . . . . . . . . . 49

4.3 .1 Experiment $2.1 \ldots \ldots \ldots \ldots \ldots$

4.3 .2 Experiment $2.2 \ldots \ldots \ldots \ldots \ldots$

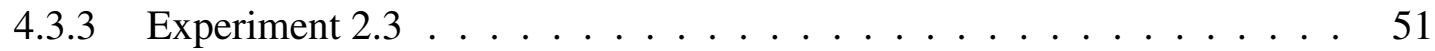

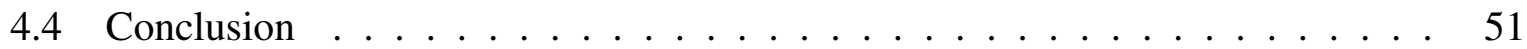

5 Study 3 - Effect of Spatial Attention 53

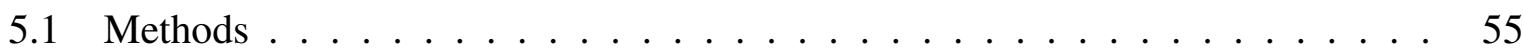

5.1 .1 Participants ........................ 55

5.1 .2 Experimental Set-Up . . . . . . . . . . . . 55

5.1 .3 Data Analysis . . . . . . . . . . . . . . . . . . 56

5.2 Results . . . . . . . . . . . . . . . . . . 57

$5.2 .1 \quad$ Experiment $3.1 \ldots \ldots \ldots \ldots \ldots$

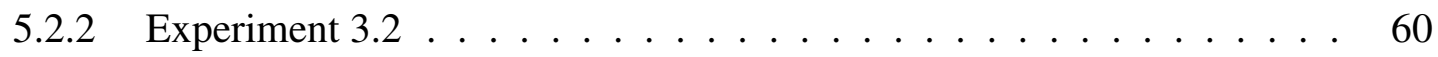

5.2 .3 Experiment $3.3 \ldots \ldots \ldots \ldots \ldots$

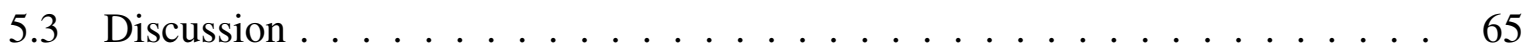

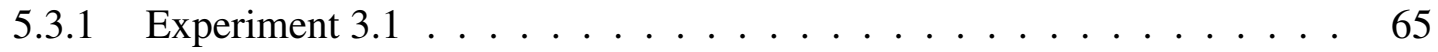

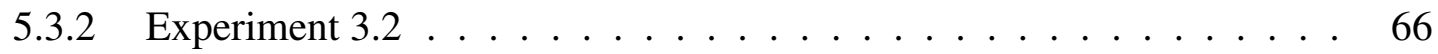

5.3 .3 Experiment $3.3 \ldots \ldots \ldots \ldots \ldots$

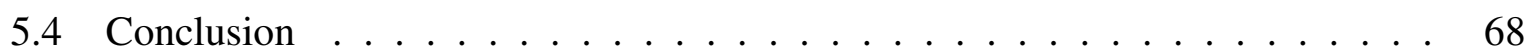

6 Study 4 - Effect of Attention 69

6.1 Methods . . . . . . . . . . . . . . . . . . . . . . . 71

6.1 .1 Participants ...................... 71

6.1 .2 Experimental Set-Up . . . . . . . . . . . . . 71

6.1 .3 EEG Recording . . . . . . . . . . . . . . . . . . 72

6.1 .4 Data Analysis ....................... 73 
6.2 Results . . . . . . . . . . . . . . . . . . 75

6.2.1 Behavioral Data . . . . . . . . . . . . 75

6.2 .2 Physiological Data . . . . . . . . . . . . . . 78

6.3 Discussion . . . . . . . . . . . . . . . . . 80

6.3.1 Experimental Discussion . . . . . . . . . . . . . . 80

6.3 .2 Methodological Discussion . . . . . . . . . . . . . . . . 84

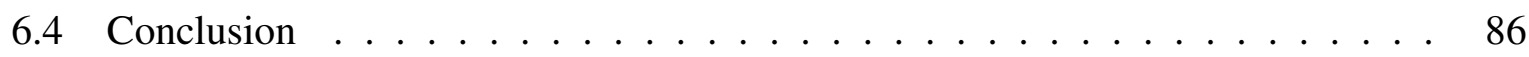

7 Study 5 - Effect of the Instructed Task

7.1 Methods . . . . . . . . . . . . . . . . . . . 88

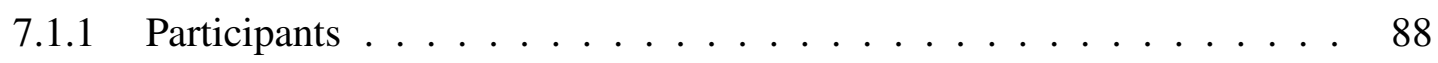

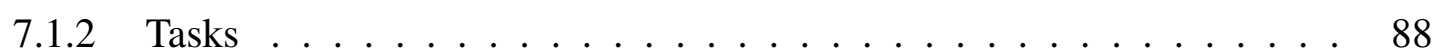

7.1.3 Experimental Set-Up . . . . . . . . . . . . . . . . . 89

7.1 .4 Data Analysis . . . . . . . . . . . . . . . . . . 89

7.2 Results . . . . . . . . . . . . . . . . . . 90

7.2 .1 Experiment $5.1 \ldots \ldots \ldots \ldots$. . . . . . . . . . . . 90

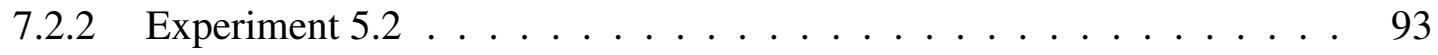

7.3 Discussion . . . . . . . . . . . . . . . . . 97

7.3 .1 Experiment $5.1 \ldots \ldots \ldots \ldots$. . . . . . . . . . . 97

7.3 .2 Experiment $5.2 \ldots \ldots \ldots \ldots$. . . . . . . . . . . . . 97

7.4 Conclusion . . . . . . . . . . . . . . . . . . 100

8 Overall Summary and Discussion $\quad 101$

8.1 Metacontrast Masking . . . . . . . . . . . . . . . . . . . 101

8.2 Studies. . . . . . . . . . . . . . . . . . 102

8.3 Results . . . . . . . . . . . . . . . . . . . . 102

8.3.1 Effects of Parameter Manipulation on Behavioral Performance . . . . . 102

8.3.2 Physiological Comparison of Types . . . . . . . . . . . . . . . . . 103

8.4 Processes Involved in Metacontrast Masking and Their Characteristics . . . . . 104

8.4.1 Revealing Two Processes . . . . . . . . . . . . . . . . . . . . 104

8.4.2 Characteristics of Processes . . . . . . . . . . . . . . . 105

8.5 Proposals for Further Research . . . . . . . . . . . . . . . . . . 106

8.5.1 Proposals for Additional Analyses . . . . . . . . . . . . . . . . 106

8.5.2 Proposals for new Experiments . . . . . . . . . . . . . . . 107

8.6 Concluding Remarks . . . . . . . . . . . . . . . . . . . . . . . 108 



\title{
1 General Introduction
}

\author{
„Das Auge sieht nur was der Geist bereit ist zu vestehen. “ \\ "The eye only sees what the mind is willing to understand."
}

Henri-Louis Bergson (1859-1941)

Perceiving the environment through vision is most fundamental for almost everyone. This quote by Henri Bergson, a French philosopher, nicely illustrates the discrepancy between physical stimulation by light rays entering the retina of the eye and the subjective perception of it. Between these two states (stimulation and perception) are a series of complex processing steps happening in the brain, which are not yet understood in their entirety. The fundamentals of visual perception are not only of interest for philosophers but for scientists as well.

\subsection{Metacontrast Masking}

When exploring the basics of visual perception only few, most simple stimuli are used to limit possibilities of interaction. A phenomenon where the visibility of one stimulus (termed target) is reduced by another stimulus (termed mask) is called visual masking and was described ever since 1910 by Stigler. By definition, the mask is spatiotemporally overlapping or contiguous to the target (Breitmeyer \& Öğmen, 2006). It was first called "metaphotischer Kontrast" and eventually evolved to metacontrast (masking). Since then it is mystery and tool at the same time (Bachmann \& Francis, 2013). On one side, the question is how these stimuli interact and how they are processed to effect this phenomenon. On the other side, it is used to deliberately manipulate the visibility of experimental stimuli, namely to a degree where conscious visibility is impaired. Thus, it is used to investigate the transition between conscious and unconscious vision and the extent of the influence of unconsciously processed stimuli. In a broader sense, masking research is in part about the role and kind of consciousness.

Today several different types of visual masking paradigms are classified differing in their stimulus features and the temporal order in which stimuli are presented. In metacontrast masking the target prevails the mask. Here, the mask has a retroactive effect on the target and therefore metacontrast masking is also called backward masking. In contrast, when the mask prevails 


\section{General Introduction}

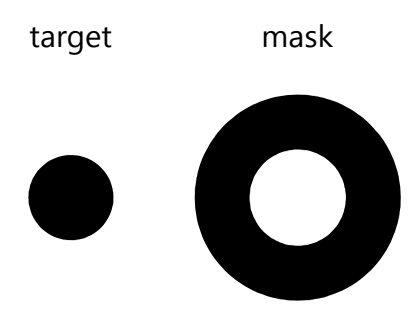

Figure 1.1: Meta- and paracontrast masking stimuli. The disc-shaped target fits into the annulusshaped mask without overlapping contours.

the target, it is called paracontrast or forward masking. In both types, contours of both stimuli are not overlapping spatially. Figure 1.1 shows exemplary stimuli with a disc as target and an annulus as mask, where the target fits into the hole of the mask without overlapping contours. When contours of target and mask stimuli are overlapping it is called pattern masking by noise or structure. (Overviews of masking types from Breitmeyer and Öğmen, 2006, chapter 2.2.) As these masking methods are not part of this dissertation they will not be discussed further. But metacontrast masking is the essential part as it is a suitable method to examine the mechanism of visual processing (Breitmeyer \& Öğmen, 2006). While the exact mechanisms of masking are still unknown, a whole series of experimental manipulations has generated profound knowledge about the consequences of experimental parameters. Specific reviews over representative studies are given in the introductions to each study.

For an effective masking timing is crucial. Here, different measurements can be used: the time between offset of the target and onset of the mask (inter-stimulus interval), the time from target onset to mask onset (stimulus-onset-asynchrony, SOA), or the time from target offset to mask offset (stimulus-termination-asynchrony). The SOA is most often used. Moreover it is regarded to be most suitable (Kahneman, 1967; Breitmeyer and Öğmen, 2006, p. 52). The degree of visibility is modulated by the time between target onset and mask onset (stimulus onset asynchrony, SOA). The lower the visibility the higher the masking effect. The curve of visibility over SOAs is called masking function. Most often two different masking functions are described, a monotonically increasing function called "Type-A", where the visibility increases with increasing SOA, and a U-shaped function called "Type-B", where the visibility is decreasing with increasing SOA and increasing with even longer SOAs (Breitmeyer \& Ganz, 1976, using the nomenclature introduced by Kolers, 1962). Which masking function is obtained depends on experimental parameters (see Chapter 3-5 for an overview) and on inter-individual differences (see Section 1.3 of this Chapter). 


\subsection{Processing Models and Theories}

Due to the complexity of the brain, it is only possible to understand its functioning by using simplified models explaining only specific aspects of sensory processing. Several models have been developed so far, but only some will be presented here that seem to be most suitable. In metacontrast masking research most often two processes were assumed to be involved in masking. The model of Weisstein, Ozog, and Szoc (1975) is based on different groups of neurons and on separate paths for target and mask processing. The target path is excitatory, slow and sustained, while the mask path is inhibitory, fast and transient. Both path consists of three neurons (n11, n12, n13, and n21, n22, n23), where neurons of one path are connected in line, and additionally there are cross-connections between paths, enabling the fast $\mathrm{n} 22$ of the mask to inhibit the slow $n 13$ of the target and therefore to disturb target processing.

In 1976 Breitmeyer and Ganz published the first version of their sustained-transient-dualchannel-model, modified in 1984 and continuously adapted since then. Its underlying theory is an accumulation of substantial results of numerous studies of which a brief summary is given here, based on Breitmeyer and Öğmen (2006, Chapter 5). For information beyond this summary, it is recommended to study this chapter in more detail.

The two main ganglion cell types in the retina are the $\mathrm{P}$ and $\mathrm{M}$ retinal ganglion cells, where the $\mathrm{P}$ cells represent $75 \%$ and the $\mathrm{M}$ cells represent $10 \%$ of the retinal ganglion cells. While $\mathrm{P}$ cells project excitations in the parvocellular layers of the lateral geniculate nucleus (LGN) primarily via the ventral path, M cells project to the magnocellular layers of the LGN primarily via the dorsal path. In the LGN parvo- and magnocellular afferents interact, where projections of the M-path precedes those of the P-path. Beyond cell types, two well-established and approved processing pathways were associated to the parvo- and magnocellular system, namely the sustained channel (or pathway) and the transient channel (or pathway). Both pathways are specialized for processing specific stimulus features, but this attribution is not dichotomous, since there are overlaps. Which pathway is activated is dependent on the task and the multidimensional aspects of the stimuli. Characterizing one channel always is meant in relation to the other channel, as no absolute attributions are valid and both channels are complementary. To characterize the sustained channel: It is more sensitive for higher spatial and lower temporal frequencies (but still responds at low temporal frequencies), has a higher contrast threshold, is activated by slow velocities, has a higher threshold to detect pattern, prefers low flicker frequencies and that high flicker frequencies, that it appears to be a sustained stimulus, it has a longer integration time, shows only in the excitatory phase, has a longer response persistence and a longer response latency, which increases with increasing temporal frequency. Overall it responds slower and has a slower signal conductivity. To characterize the transient channel: It is more sensitive for low spatial and high temporal frequencies, it is more selective for move- 


\section{General Introduction}

ments and fast velocities, has a lower contrast threshold, has a lower threshold to detect flicker and is more sensitive for higher flicker frequencies, it has a shorter integration time, shows multiphasic oscillations, in which excitation and inhibition are alternating, and it has a shorter response latency. Overall it responds faster, has a faster signal conductivity, and all mechanisms are enhanced with eccentricity.

In contrast to one-channel-models, in a dual-channel-model it is assumed that a stimulus activates more than one process. Thus, not only the progress in time is important but in addition the interaction of both processes. Based on the theory described above the transient channels signal mainly information of the location and the presence of the stimuli or the rapid change of their position (like displacement or movements), while the sustained channels signal information about pattern aspects of the stimulus like brightness, contrast, contour, and about stationary or slowly moving stimuli. In the sequence of target and mask in a metacontrast masking paradigm both stimuli activate both channels by their own. Channel activations can be inhibited by activations in the same channel, or by activations of the other channel. Within one channel inhibition is realized via center-surround antagonists of the receptive field (intra-channel inhibition). Between two types of neurons a reciprocal inhibition occurs (inter-channel inhibition). The masking effect occurs either due to intra-channel inhibition (mainly in sustained channels), due to interchannel inhibitions (mainly transient-on-sustained channel inhibition), or due to an activation of both channels as both stimuli patterns overlap spatially (as in pattern and noise masking). It is assumed that in metacontrast masking the transient activation of the mask interacts with the early sustained activation of the target via the transient-on-sustained-channel inhibition.

This basic Sustained-Transient-Dual-Channel-Model was updated in cooperation with Ö $\breve{g}$ men in order to take dynamics in visual processing into account (Breitmeyer \& Öğmen, 2006), the retino-cortical dynamics (RECOD) model was developed (Breitmeyer \& Öğmen, 2000). Here, the concepts of feedforward and feedback signal processes were used, where feedforward signals deliver a stimulus-dependent activity via afferent paths, and feedback signals process this activity and transform it into a percept-dependent activity via efferent paths, synthesizing the perception. To keep both parts well balanced the dynamic characteristics are described in three phases: When a stimulus signal arrives the feedforward-dominant phase is activated and the strong afferent signals sustainably energize the feedback loops (corresponds to the sustained channel). Now these afferent signals were decayed to a lower level in order to strengthen the feedback-dominant phase where perceptual synthesis is achieved. When the stimulus input changes, the recurrent feedback signals were inhibited fast and transiently in order to strengthen again the afferent activity. This is called the reset phase (that corresponds to the transient channel). Therefore, the reset phase corresponds to the transient-on-sustained inter-channel inhibition, and in addition with the sustained-on-transient inter-channel inhibition (feedback- 
dominant phase), this recurrent inhibition enables a dynamic balance between figural synthesis and reset. Without this recurrent inhibition, noise would often lead to a reset and thus destroy the figural synthesis. Based on the knowledge that form and surface are processed at different speeds and by different groups of neurons, the parvocellualar sustained path is unlumped into two networks where one network processes contour information and the other network the surface-brightness information. A stimulus input (for example a brief flash) elicits three processes, a fast transient activation, a slower sustained contour process and a still slower sustained surface/brightness process. Depending on the SOA the transient activity of mask inhibits either the contour or the surface sustained activity.

Apart from models, detailed descriptions and scrutinizing interpretations of results help to understand the basic mechanisms behind metacontrast masking. Neumann (1978) introduced the hypothesis that the U-shape of metacontrast masking function is a result of two monotonic components (remastered and translated version: Neumann \& Scharlau, 2006). One component is responsible for the decreasing branch (in short to intermediate SOAs), and one component for the increasing branch (in intermediate to long SOAs), which can be both manipulated independently by different parameters. The slope of the decreasing branch can be manipulated for example by the lowering of the target-to-mask ratio (for a review see Chapter 3), whereas the the slope of the increasing branch is manipulated in general by higher signal processes. Reeves (1982) confirmed Neumann's theory. He as well showed that the metacontrast masking function is a construct of two monotonic functions of competing processes associated with percepts of temporal integration or succession. The less target and mask are integrated the more the masking function decreases and the higher the masking effect becomes. In even longer SOAs stimuli are perceived in succession, leading to an increase of the masking function. Dixon and Dilollo (1994) described the phenomenon of temporal integration as the result of the apparent simultaneity of two succeeding stimuli. The temporal gap between both stimuli is bridged by the visible persistence of the first stimulus. Depending on how long the first stimulus is phenomenally visible after its physical offset, both stimuli are perceived integrated or segregated. Until today the perception of integration and segregation of successive stimuli is examined in the context of vision research (e.g. Akyürek, Schubö, \& Hommel, 2010; Francis \& Cho, 2008; Geerligs \& Akyürek, 2012; Samaha \& Postle, 2015; Wutz, Weisz, Braun, \& Melcher, 2014).

\subsection{Inter-Individual Differences}

The analysis of inter-individual differences is not common in psychological research, although it reveals interesting phenomena that can be ascribed to the complexity and non-generality of the brain's functioning. Furthermore, being unaware of the variability between datasets may 


\section{General Introduction}

lead to misinterpretations of the results. Inter-individual differences were reported since the beginnings of metacontrast masking research. For example, Eriksen, Becker, and Hoffman (1970) reported considerable variability in the individual performance curves in the decreasing branch. Weisstein (1970) described a large variation across participants and suggests to consider them individually rather than to average datasets (Weisstein, 1972). Furthermore, Neumann (1978) refers to several studies, which report inter-individual differences in either the decreasing or increasing branch of the U-shaped masking function depending on which experimental parameters are manipulated. In a more recent study Albrecht, Klapötke, and Mattler (2010) found that not only experimental settings condition the masking type but that there are inter-individual differences despite identical experimental settings as well. They could show that despite identical experimental settings some participants showed a Type A function and others a Type B function. These inter-individual differences turned out to be stable for each participant over several months (Albrecht \& Mattler, 2012a) and with different instructions (Albrecht \& Mattler, 2012b).

But not only objective performance differs across participants but the subjective perception as well. The stimulus dimension, along which the perceptual judgment about the target is made, is called criterion content (Kahneman, 1968). The criterion content depends on the task requirements (Breitmeyer et al., 2006). As metacontrast masking depends on the phenomenal appearance of the target (Weisstein, 1972), objective inter-individual differences also result from subjectively different contents (Bachmann \& Francis, 2013). Albrecht and Mattler (2012b) showed that the Response Bias $C_{R}$ (based on the Signal Detection Theory by Macmillan \& Creelman, 2005) can be equally used to identify Type A or Type B observers as the Discrimination Sensitivity $d^{\prime}$. Here, the congruency of the stimuli is important for Type A observers, but it is unimportant for Type B observers. In addition, participants report phenomenological percepts they are using to deal with the task: Type A observers report to see and use percepts of apparent motions like rotating or magnifying movements during the target-mask-transition. Type B observers report to see and use negative afterimages in form of the target inside of the mask. This percept is sometimes reported as well by Type A observers but was seldom used by them to identify the shape of the target.

The integration or segregation of two succeeding stimuli (introduced in Section 1.2) are linked to the phenomenal perception that target and mask stimuli are conjoint or disjoint (Dixon \& Dilollo, 1994; Neumann \& Scharlau, 2006; Reeves, 1982). So, different masking functions go along with different phenomenological perceptions as well as with inter-individual differences on either the integration or segregation process. Therefore, it is logically consistent to say that negative afterimages inside and during the mask stimulus in short SOAs as reported in Albrecht and Mattler (2012a) are a strong evidence for an integration process, as stimuli are perceived 
temporally simultaneously as one conjoint percept. Additionally, the perception of apparent motion indicates a successive perception of stimuli and thus indicates a segregation process. Assuming that different stimulus aspects were processed differently weighted by different participants, implications can be made about the influence of a specific stimulus parameter on the processing of specific stimulus aspects. A gradual assignment on the basis of the involved neural processes will be used (as in Albrecht \& Mattler, 2016) to substantiate the assumption of an integration and a segregation process, influencing visual perception in metacontrast masking designs.

\subsection{Aim and Outline of Studies}

Although research on metacontrast masking has long history it is still unknown how exactly the interaction of target and mask stimulus causes the phenomenon of masking. This dissertation aims to contribute to the understanding of the fundamentals of metacontrast masking and addresses the question, which processes are involved and which influence they have on masking functions under specific experimental parameters. For this, individual differences are addressed to make assumption about the characteristics of these processes.

Five studies were conducted. All have in common that the same stimuli in the same paradigm were used, and that they include a standard experiment were individual differences were determined using a discrimination task. They differ in the manipulation of different experimental parameters, partly in the task to be fulfilled, and one study has an additional measuring method. These five studies were designed as they reveal crucial information necessary to characterize the processes involved in metacontrast masking functions. In the first three studies it was examined how specific experimental parameters influence inter-individual differences to characterize these processes. In Study 1 (page 13 et seqq.) the influence of different stimulus energies, in Study 2 (page 35 et seqq.) the influence of eccentricity and stimulus size, and in Study 3 (page 53 et seqq.) the influence of spatial attention was examined. For Study 4 (page 69 et seqq.) a neurophysiological method was used to further characterizes when and how these individual differences occur in order to determine the differences in signal processing, which effect metacontrast masking. And in Study 5 (page 87 et seqq.) the specifics of the criterion content were examined and how they could explain different processing. 



\section{General Methods}

All studies share common methods described in this chapter apply to each experiment, unless otherwise stated. Any variations and additions were described in the specific method sections of each study.

\subsection{Participants}

In all experiments participants had normal or corrected-to-normal vision, were students, and were naive to the experimental setup. They were paid by $7.00 €$ per hour for their participation in behavioral sessions and 7.50 € per hour for their participation in physiological sessions. All experiments were approved by the ethical commission of the University of Göttingen.

\subsection{Stimuli}

The target stimulus was either a black filled square or a black filled diamond with a visual angle of $1.7^{\circ}$ (outer diameter of most distant corners, Figure 2.1a, page 10). The outer shape of the mask stimulus was also either square- or diamond-shaped, allowing congruent (target and mask have same outer shapes) and incongruent pairs (target and mask have different outer shapes), with a visual angle of $2.7^{\circ}$ (outer diameter of most distant corners). The inner contours of the mask stimuli was a star-shaped pattern that fitted neatly around the contours of both target stimuli but leaving one pixel blank between contours (which corresponds to $0.02^{\circ}$ of visual angle). All stimuli were black $\left(0.03 \mathrm{~cd} / \mathrm{m}^{3}\right)$ presented on a light gray $\left(72.3 \mathrm{~cd} / \mathrm{m}^{3}\right)$ background in the center of the monitor. Congruent trials and incongruent trials as well as square and diamond shaped targets were balanced over a session and over an experiment.

\subsection{Experimental Set-Up}

Stimuli were presented with Presentation ${ }^{\circledR}$ (Presentation (version 16.1) [Computer software], 2012) on a ViewSonic Professional Series G90fB monitor with a display resolution of 1024 x 768 pixel and a vertical screen refresh rate of $85 \mathrm{~Hz}$. The viewing distant was $100 \mathrm{~cm}$. To keep this 
(a)

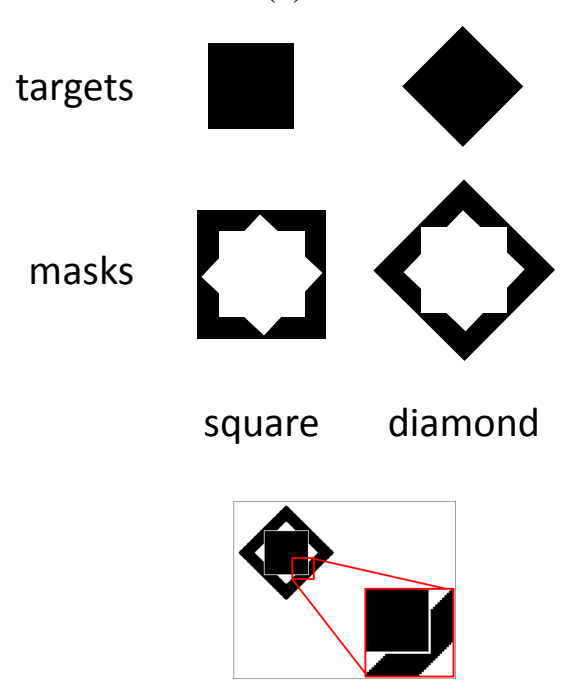

(b)

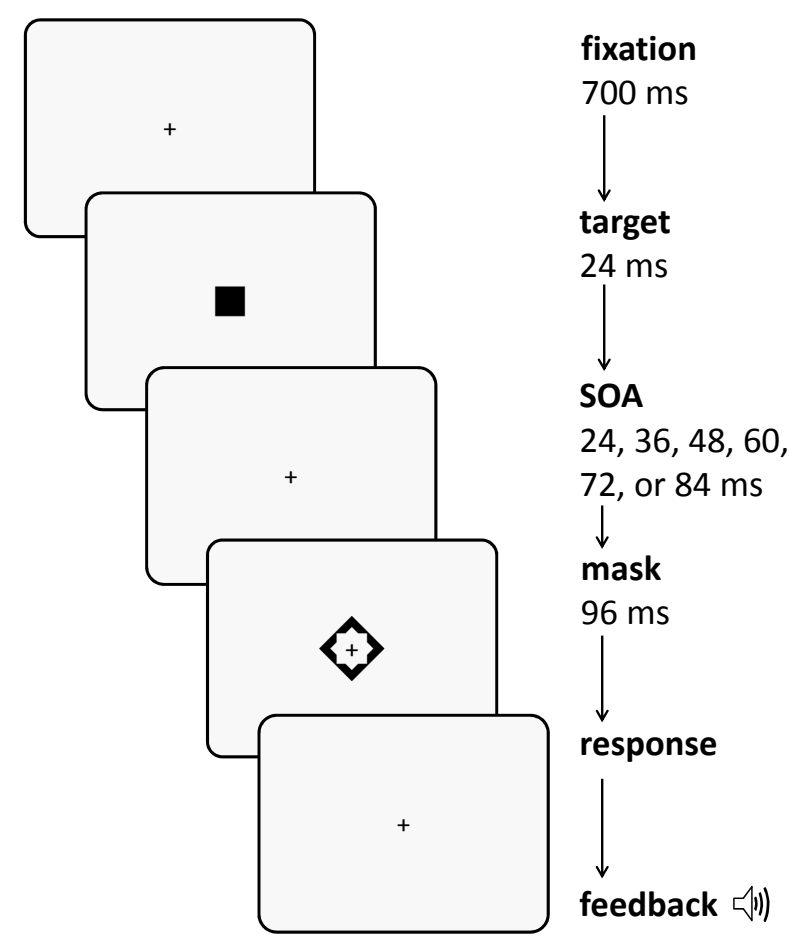

Figure 2.1: (a) Stimuli and (b) trial course.

distant constant across participants and to stabilize the head position, a chin rest was used. During the whole trial a fixation cross was presented in the center of the monitor, which had to be fixated all the time by the participant. The target was presented $700 \mathrm{~ms}$ after beginning of the trial for $24 \mathrm{~ms}$, while the mask was presented for $96 \mathrm{~ms}$. The SOA was varied randomly between 24 and $84 \mathrm{~ms}$ in $12 \mathrm{~ms}$ steps (Figure 2.1b, page 10). The inter-trial-interval varied randomly between 700 and $1500 \mathrm{~ms}$. Participants were instructed to wait for the disappearance of the mask to make their response. When an experiment had to be split into several sessions, each was conducted on separate days. After each erroneous response an auditory feedback $(440 \mathrm{~Hz}$, $150 \mathrm{~ms}$ ) was given via loudspeaker boxes placed next to the monitor. If the response was given too quickly (less than $500 \mathrm{~ms}$ after mask onset) a distinct auditory feedback (555 ms) was given, to remind the participant to delay their response.

Experimental sessions were conducted in a darkened room with the monitor as only illuminant. At the beginning of each experimental session, additional trials were presented, serving as practice trials to enable the participant to get familiar with the task and to adjust to the darkness. They were not used for analysis. The examiner stayed in the room until practice trials were finished and all questions were answered. After each session the examiner interviewed the participants how difficult they judged the task, how they solved the task (and if they had 
strategies they used), if they paid attention to the second stimulus as well, how well they managed to fixate the fixation cross, if they were motivated over the whole session to do the task as best as possible, if they were tired, and if they had any problems during the session. After the last session of each study additionally participants were asked if there were trials where they perceived a light picture or some kind of afterglowing of the first stimulus, and if there were trials were they perceived a movement, a rotation, or expanding in the sequence of both stimuli. When participants answered one or both with yes, they had to judge how often they perceived this and if they used this perception for shape identification of the first stimulus.

\subsection{Data Analysis}

\subsubsection{Signal Detection Theory}

Data were analyzed using the signal detection theory by Macmillan and Creelman (2005), resulting in values for the Discrimination Sensitivity $d^{\prime}$ and Mask Bias $C_{M}$ for each participant and SOA (using MATLAB R2013a (version 8.1.0.604) [Computer software], 2013). To this end the proportion of "square" reports to square shaped targets were defined as hits, and "square" reports to diamond shaped targets were defined as false alarms. To prevent confounding, Sensitivity and Response Biases $C_{R}$ were computed separately for each mask and subsequently averaged across masks (Vorberg, Mattler, Heinecke, Schmidt, \& Schwarzbach, 2003). To assess the tendency for responding according to the mask, first the signal detection's response criterion $C_{R}$ was calculated separately for each mask with positive values reflecting a tendency to respond "diamond"and negative values reflecting a tendency to respond "square". Subsequently, the Mask Bias $C_{M}$ was computed as the difference $C_{M}=0.5\left(\mathrm{C}_{\text {diamond mask }}-\mathrm{C}_{\text {square mask }}\right)$. Thus, positive values of Mask Bias $C_{M}$ reflect a tendency to respond according to the shape of the mask, while negative values signify a tendency to respond contrary to the shape of the mask stimulus. Plotting $d^{\prime}$ over SOA results in a masking function, which describes the time course of metacontrast masking. Likewise, $C_{M}$ is plotted over SOA to describes the course of the bias associated to the masking.

\subsubsection{Factor Analysis}

The factor analysis is a data reduction procedure where some latent variables (factors) are deduced from many different manifest variables, obtained in empirical observations (Klopp, 2010). To estimate the number of underlying variables, that are sufficient to describe the individual variability in masking functions, a maximum likelihood exploratory factor analysis with subsequent Varimax Rotation was conducted (using R Core Team (version 3.0.1) [Com- 


\section{General Methods}

puter software], 2013). In addition, factor scores for each participant by regression were estimated, giving Thompson's scores. Factor loadings represent the correlation between the variable (which will be the SOA in the presented studies) and the factor. Factor scores represent the relationship of individual datasets to the factors. The cumulative variance tells how much variability can be explained by all factors. Cummunalities represent how much variance can be clarified by both factors for each variable (SOA). The sum of squares states how much variance can be clarified. Values greater than one mean that the factor is suitable to explain the data.

To test if $C_{M}$ values behave in the same manner as $d^{\prime}$ values, an analysis of covariance (ANCOVA) was calculated using the $C_{M}$ values as dependent variable and the factor scores as covariates (using R Core Team (version 3.0.1) [Computer software], 2013). In addition, using a linear model, the correlation of individual $d^{\prime}$ and $C_{M}$ values with the individual factor scores were calculated. The slope of the regression, the slope parameter $\beta$, is plotted for each SOA, representing the impact of this correlation. Positive values mean the higher the factor score the higher $d^{\prime} / C_{M}$, negative values mean the higher the factor score the lower $d^{\prime} / C_{M}$. 


\section{Study 1 - Effect of the Ratio of Target Duration to Mask Duration}

The effect of of metacontast masking depends crucially on temporal, spatial, and object-specific experimental parameters. Object-specific parameters are for example stimulus shape, size, or luminance. Spatial parameters concern the presentation position in the field of view, namely the degree of eccentricity - if stimuli are presented in the fovea or in the periphery. But most important are temporal parameters. Here, the time range between the onset of the first stimulus (target) and the onset of the second stimulus (mask), the stimulus-onset-asynchrony (SOA), place a crucial role (Di Lollo, Mühlenen, Enns, \& Bridgeman, 2004; Kahneman, 1967). In addition, the durations of the presented stimuli influence metacontrast masking, and in fact independently of the object-specific parameters - the variation of the duration of a light flash (Stewart \& Purcell, 1974) as well as a solid geometric figure (Breitmeyer, 1978).

Back in 1885, Bloch experimented with the applied duration of light and the perceived intensity of a luminous body, up to its invisibility with specific parameters. He found a reciprocity of light and duration, later known as Blochs' law: Response = Intensity x Time (Kaiser, 2016). That is, for a constant effect doubling the intensity requires halving the duration of light exposure. Or, considering both factors as a product, the response can be predicted.

Applying Bloch's law to metacontrast masking research, the stimulus detection performance depends on the interaction of the stimulus' luminance and stimulus' presentation duration. However, as in the masking paradigm the mask stimulus influences target's detection, both stimuli have to be regarded jointly instead of each for its own. It is common practice to implement the relation of characteristics of both the target and the mask, in a target-to-mask-ratio (T/M ratio) (some representatives addressed below). To calculate the T/M ratio the value, e.g. of luminance, of the target is divided by the value of the mask, resulting in 1 for equal intensity, $>1$ when the mask is brighter than the target, and $<1$ when the target is brighter than the mask. Stewart and Purcell (1974) varied the luminance of the mask while keeping the target's luminance constant. Fehrer and Smith (1962) varied the luminance of the target while keeping the mask's luminance constant. Both report a variation in resulting masking functions when the ratio is changed. Weisstein (1972) used a disc as target stimulus and an annulus as mask stimulus, and varied the luminance of the target while keeping the luminance of the mask constant. 


\section{Study 1 - Effect of the Ratio of Target Duration to Mask Duration}

She reported a systematical shift of the masking function in specific $\mathrm{T} / \mathrm{M}$ ratios, ranging from 1 to 0.0625 . Namely, when the ratio is 1 a U-shaped masking functions is obtained. When the ratio decreases the minimum of the U-shaped masking function shifts toward shorter SOAs, becoming more J-like. In the smallest ratio the function is even weak monotonic.

Breitmeyer (1978) adopted her approach (and stimuli) on stimulus duration manipulations. He varied the mask duration while keeping the target duration constant. To have comparable ratio values he took the mask-to-target-ratio (M/T ratio), ranging from 2 to 0.0625 . With the shortest mask duration of $1 \mathrm{~ms}$ no masking occurred. But with increasing masking durations an increasingly deeper U-shape masking function emerged. At masking durations of $16 \mathrm{~ms}$ and $32 \mathrm{~ms}(\mathrm{M} / \mathrm{T}$ ratio $=1$ and 2 , respectively), the masking effect in the shortest SOA enhances, resulting in a more monotonically increasing masking function.

Macknik and Livingstone (1998) went a step further and varied the target as well as the mask durations. For their experiment they used bar stimuli and T/M ratios between 0.222 and 2.8, using target durations of 20,40, 90, and $140 \mathrm{~ms}$ and mask durations of 50 and $90 \mathrm{~ms}$. The masking function with a $\mathrm{T} / \mathrm{M}$ ratio of 0.222 is monotonically increasing, while those with a $\mathrm{T} / \mathrm{M}$ ratios of $0.4,0.8$, and 1 show $\mathrm{U}$-shaped functions.

The previous mentioned studies varied either one factor of Bloch's law, the light intensity or perceived stimulus brightness, or the other factor of Bloch's law, the duration of light or stimulus exposure, for the target and/or the mask stimulus. Di Lollo et al. (2004) regarded this issue and manipulated target and mask duration as well as the perceived brightness of the stimuli to decouple duration and brightness. They increased the presentation duration when the corresponding luminance was decreased to gain a perception judged equally bright for all stimuli, independent of the presentation duration. The used stimuli were diamond-shaped outlines, the target smaller than the mask with one of four possible truncated corners, whose position has to be detected. They found that the detection performances in the brightness-matched condition has a U-shaped function with increasing target duration (as the inter-stimulus-interval is kept zero this refers to the SOA as well) and a monotonically decreasing function with increasing mask duration. But no statements could be made how masking functions behave when the SOA is varied for the different target and mask durations.

The present study aims to determine the characteristics of the processes involved in metacontrast masking to clarify how they effect inter-individual differences and how these processes behave when stimulus presentation durations are manipulated. By varying the T/M ratio the processes assumed to effect Type A or Type B masking functions become differently involved and findings how inter-individual differences become effected by this variation indicate whether inter-individual differences are referable to gradual differently involved mechanisms. The study is composed of three experiments: In Experiment 1.1 the SOA is varied in a metacontrast 
paradigm. It is expected to replicate previous studies by Albrecht and Mattler (2012b) and obtain individually different masking functions for each participant. In Experiment 1.2 the same paradigm is used and, in addition to the SOA, the presentation durations of the target and the mask were manipulated to examine how the $\mathrm{T} / \mathrm{M}$ ratio effects the individual masking functions. It is expected that the minimum of the masking function shifts toward shorter SOAs in all participants the larger the T/M ratio becomes. By replicating effects shown by Weisstein (1972) and Breitmeyer (1978) the used stimuli and paradigm can be confirmed. Furthermore, data of Experiment 1.1 were associated with data of the Experiment 1.2 to compare underlying mechanisms of inter-individual differences and of the T/M ratio effects. Depending on how these mechanisms behave in different $\mathrm{T} / \mathrm{M}$ ratios conclusions about their characteristics can be drawn. Finally, the Experiment 1.3 was a replication of Experiment 1.1 to examine how stable the inter-individual masking functions are related to training and context effects. It is expected to obtain masking functions comparable to Experiment 1.1.

\subsection{Methods}

\subsubsection{Participants}

Twenty healthy participants (10 male) participated in this experiment. One female participant had to be excluded for analysis as she misunderstood the task. The mean age of the remaining nineteen participants was $\mathrm{M}=24.6(\mathrm{SD}=3.8)$, ranging from 20 to 36 .

\subsubsection{Experimental Set-Up}

The experiment is composed of three experiments with nine session in total, lasting 30 to $60 \mathrm{~min}$ each. Experiment 1.1 and 1.3 were run as shown in Figure 2.1b (page 10). In Experiment 1.2 target and mask durations were varied between 24, 48, 96, or $192 \mathrm{~ms}$, while the mask was presented at least as long as the target, resulting in ten duration conditions. Thus, the T/M ratio was varied between 0.125 and 1 . In all sessions they had to fulfill the target identification task.

Experiment 1.1 includes the first two sessions. The first half of the first session served as training allowing the participant to get familiar with the procedure and to develop a strategy for solving the task. All analyses of the Experiment 1.1 include only data from the second half of the first session and the entire second session, resulting in 720 trials for analysis, 120 trials per SOA. Experiment 1.2 includes sessions three to eight, encompassing 5760 trials, 96 trials for each SOA-duration condition. Experiment 1.3 includes session nine, being composed of 720 trials, 120 trials per SOA. 


\subsubsection{Data Analysis}

With the data of Experiment $1.1 \mathrm{a}$ factor analysis was conducted as described in Chapter 2.4. Data of Experiment 1.2 were averaged across sessions and for each condition. Statistical analyses were run with target duration as independent variable. In a first step, an analysis of variances (ANOVA) was conducted to examine the overall effects of target and mask duration. In a second step, to test for the effect of individual differences, a full factorial repeated measures analysis of covariance (ANCOVA) was conducted with the within-subject factors SOA and target/mask duration, and the two continuous between-subject covariates Type A score and Type B score obtained in Experiment 1.1. ANCOVA models included all pure within-subject effects, the main effect for each covariate, and the interactions of each covariate with all within-subject effects. Thus, all effects including an interaction of both covariates were excluded. The slope of the regression of $d^{\prime}$ and $C_{M}$ values with the factor scores, the slope parameter $\beta$, is plotted for each SOA, representing the correlation between $d^{\prime}$ and $C_{M}$ values and the factor scores. Statistical analysis was conducted using R (R Core Team (version 3.0.1) [Computer software], 2013).

\subsection{Results}

\subsubsection{Experiment 1.1}

\section{Discrimination Sensitivity and Mask Bias}

The masking functions of each individual participant are shown in Figure $3.1(\bullet$, page 17). There is substantial inter-individual variability with regard to the slope and the level of masking functions including Type A (monotonical increasing, e.g. number 8) and Type B (monotonic decreasing, e.g. number 3). Furthermore, the performance level differs among participants, including different levels in each type, and additionally so called underachievers (e.g. number 6) and overachievers (e.g. number 18), in which performance is equal for all SOAs. Due to this inter-individual variability the average over all datasets results in an inconclusive function. Accordingly, an one-way repeated-measures ANOVA showed no significant effect of SOA on Sensitivity $d^{\prime}(F(5,90)=0.50, p=.543)$.

$C_{M}$ values represent the bias to respond according to the shape of the mask (positive values) or contrary to the shape of the mask (negative values). Individual data for Mask Bias $C_{M}$ as function of SOA are shown in Figure $3.2(\bullet$, page 18). Visual inspection of the data suggests that, typically, decreasing Mask Bias functions found in participants with Type A masking functions (e.g. number 8). Whereas slightly negative values for all SOAs resulting in a horizontal function are typically found in Type B masked participants (e.g. number 3). Overall Mask Bias decreases 


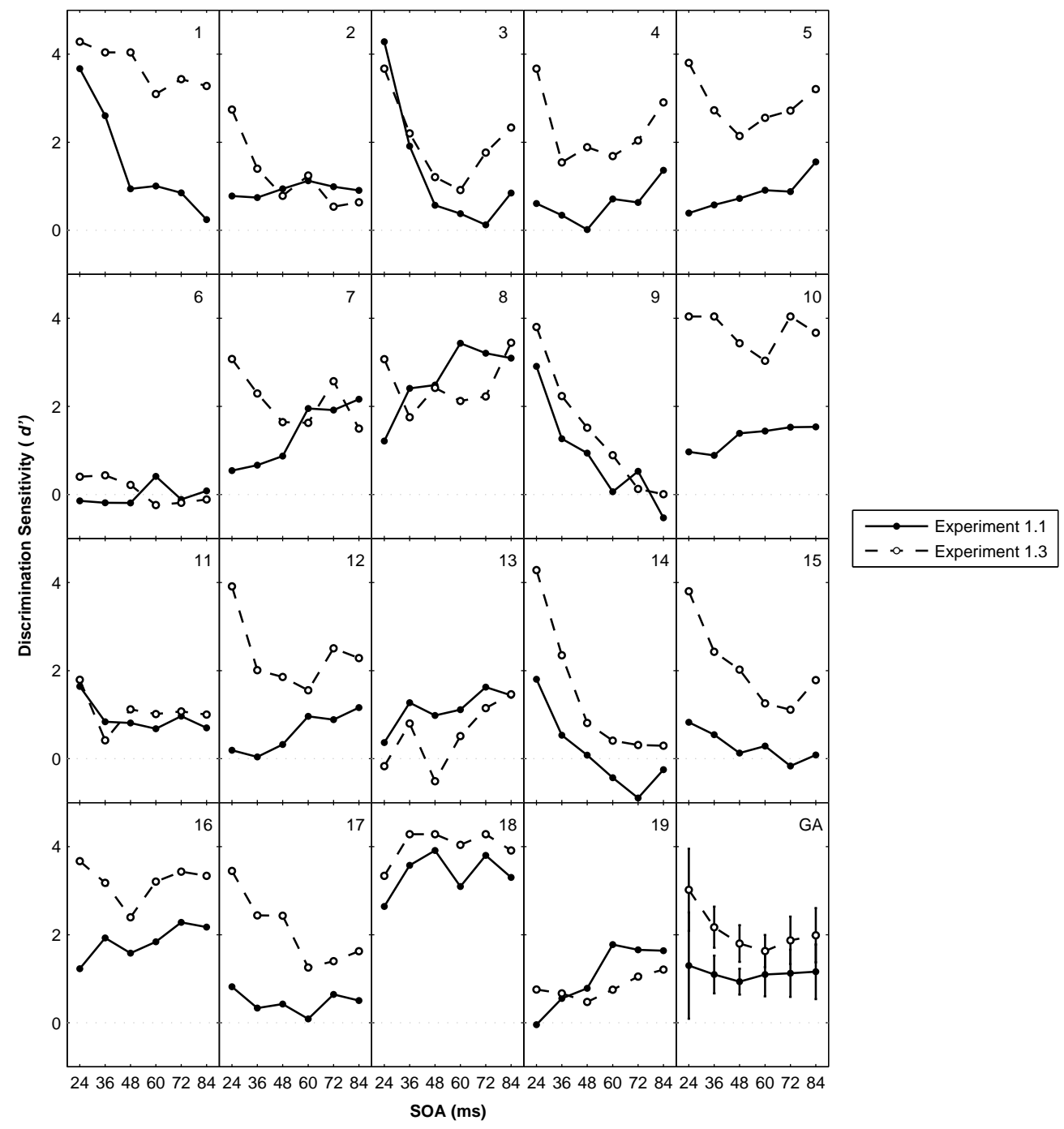

Figure 3.1: Experiment $1.1(\bullet)$ and 1.3. (०). Discrimination Sensitivity $d^{\prime}$ (individual plots [1-19] and grand average [GA]). Error bars represent the within-subject standard error (Loftus \& Masson, 1994).

with increasing SOA (GA panel). This effect proved significant in a one-way repeated-measures $\operatorname{ANOVA}(F(5,90)=11.47, p<.001)$.

\section{Factor Analysis}

The maximum likelihood factor analysis revealed two factors with eigenvalues $>1$, which together explain $93 \%$ of total variance $\left(\chi^{2}(4)=3.2, p=.51\right.$, see Table 3.1, page 20). In contrast, the one-factor solution only explains $64 \%$ of total variability, and thus fits the data only poorly $\left(\chi^{2}(9)=45.5, p<.001\right)$. A third factor would have an eigenvalue considerably smaller than one (eigenvalue $=0.07$ ), and would explain only $1 \%$ of variability (in total $94 \%$ ). Therefore, two factors are most appropriate for the data. Loadings of Factor 1 are increasing over SOAs, 


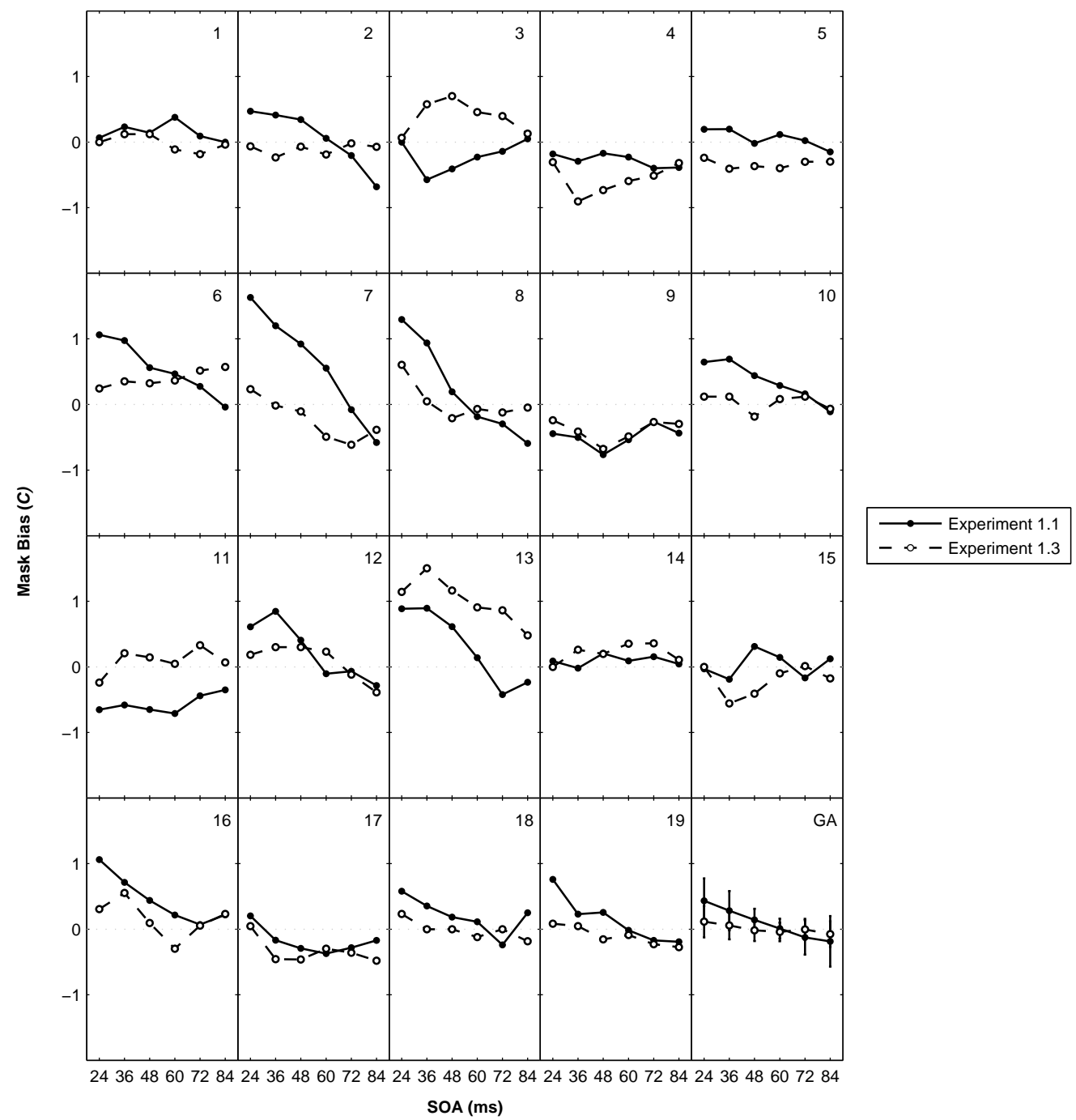

Figure 3.2: Experiment 1.1(•) and 1.3. (०). Mask Bias $C_{M}$ (individual plots [1-19] and group average [GA]). Error bars represent the within-subject standard error (Loftus \& Masson, 1994).

while loadings of Factor 2 are decreasing over SOAs (Figure 3.3, page 19). As Factor 1 loads high at long SOAs but low at short SOAs, it describes the process leading to a Type A masking function and therefore will be named "Factor A"subsequently. By contrast, Factor 2 loads low at long SOAs but high at short SOAs, thus, describing the process leading to a Type B masking function, and will be named "Factor B"subsequently. Figure 3.3 also shows the factor scores for each participant. Some participants have high scores on Factor A and low scores on Factor B, others vice versa. Still others have high and low scores on both factors, respectively. 


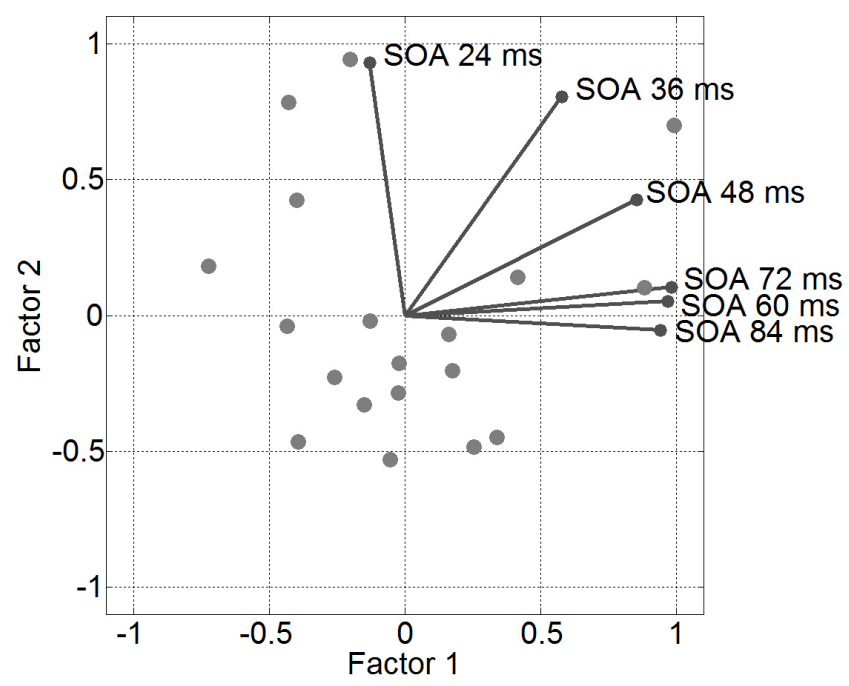

Figure 3.3: Experiment 1.1. Factor analysis - biplot with factor loadings (-) for each SOA and factor scores for each participant $(\bullet)$. (Please note that scales refer to factor loadings.)

\section{Correlation of Mask Bias With Individual Factor Scores.}

To investigate a possible relation of Mask Bias $C_{M}$ with individual masking performance, an ANCOVA with SOA as repeated-measures independent variable and the individual factor scores as covariates was conducted. There is a significant main effect of SOA $(F(5,80)=20.22$, $p<.001)$, and significant interactions of SOA with Factor A scores $(F(5,80)=9.85, p<.001)$ and of SOA with Factor B scores $(F(5,80)=6.04, p=.005)$. There is no main effect for Factor A scores $(F(1,16)=3.62, p=.075)$ and Factor B scores $(F(1,16)=1.61, p=.223)$. The correlation between factor scores and $C_{M}$ values for each SOA (slope parameters $\beta$ ) is plotted in Figure 3.4 (page 20). The slope between $C_{M}$ values and Factor A scores is positive for short SOAs and decreasing with increasing SOA (approaching zero), meaning that a high factor score on Factor A leads to a high positive Mask Bias in short SOAs. The slope with Factor B scores is mirrored-it is negative with short SOAs and is increasing with increasing SOA (approaching zero), meaning that high a factor score on Factor B leads to a low Mask Bias for short SOAs. Both lines intersect between the $60 \mathrm{~ms}$ and $72 \mathrm{~ms}$ SOA.

\subsubsection{Experiment 1.2}

\section{Discrimination Sensitivity}

The mean masking performance in Experiment 1.2 as function of SOA, target duration and mask duration is shown in Figure 3.5 (page 21). Masking had none effect when the target was presented for $96 \mathrm{~ms}$ as well as for $192 \mathrm{~ms}$ as the performance of all participants were at 
Table 3.1: Experiment 1.1. Factor analysis - factor loadings for each SOA.

\begin{tabular}{rccc}
\hline SOA & Factor 1 & Factor 2 & communalities \\
\hline $24 \mathrm{~ms}$ & -0.130 & 0.933 & 0.888 \\
$36 \mathrm{~ms}$ & 0.577 & 0.807 & 0.985 \\
$48 \mathrm{~ms}$ & 0.852 & 0.428 & 0.909 \\
$60 \mathrm{~ms}$ & 0.967 & 0.053 & 0.939 \\
$72 \mathrm{~ms}$ & 0.980 & 0.106 & 0.971 \\
$84 \mathrm{~ms}$ & 0.940 & -0.054 & 0.886 \\
\hline sum of squares & 3.854 & 1.723 & \\
proportion variance & 0.642 & 0.287 & \\
cumulative variance & 0.642 & 0.930 & \\
\hline
\end{tabular}

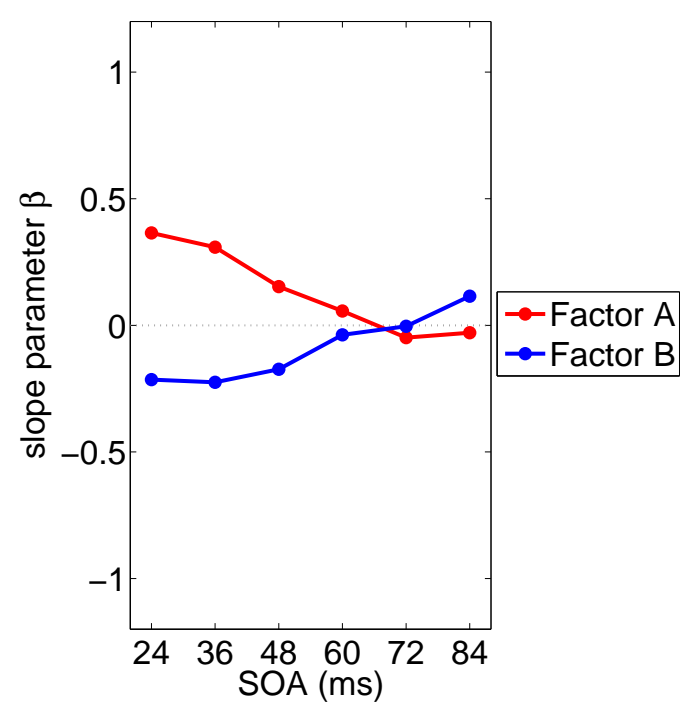

Figure 3.4: Experiment 1.1. Slope parameter $\beta$ over SOAs, with Mask Bias $C_{M}$ values correlated with Factor A scores and Factor B scores.

maximum level for all SOAs. Therefore, analyses is limited on target durations of $24 \mathrm{~ms}$ and $48 \mathrm{~ms}$. Individual masking functions with all conditions are shown in Figure 3.6 (page 22).

Target Duration 24 ms. With target duration of $24 \mathrm{~ms}$ performance improves with decreasing mask duration (main effect mask duration: $F(3,54)=12.08, p<.001$ ) and follows a Type B masking function (main effect SOA: $F(5,90)=17.73, p<.001$ ). However, graphs of different mask durations have distinct time courses (2-way-interaction mask duration $\mathrm{x}$ SOA: $F(15,270)=10.11, p<.001)$. With increasing mask duration the slope of the masking function becomes more negative and the minimum of the masking function shifts towards shorter SOAs. The effect of mask duration is most prominent at short SOAs. 


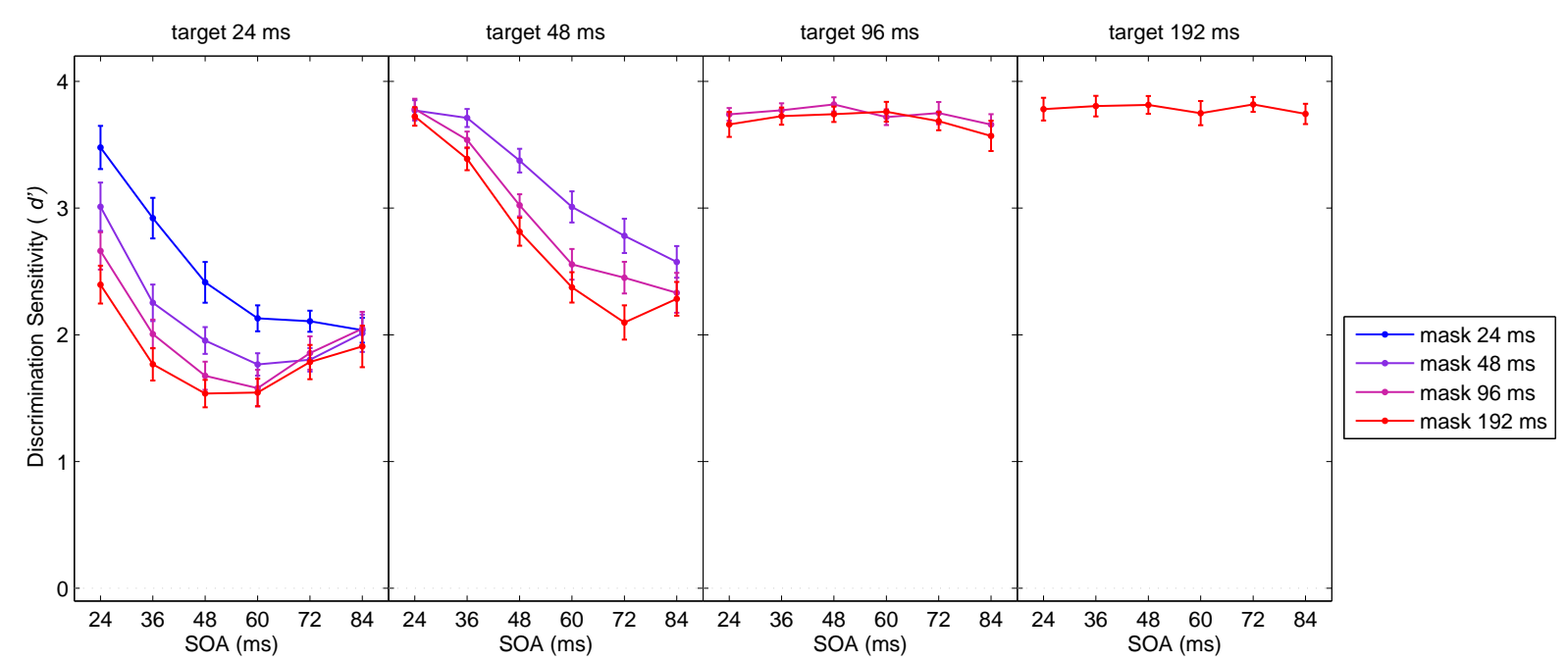

Figure 3.5: Experiment 1.2. Discrimination Sensitivity $d^{\prime}$ (group average). Each diagram has one fixed target duration, and all corresponding mask durations are presented by different graphs (see legend). Error bars represent the within-subject standard error (Loftus \& Masson, 1994).

Target Duration $48 \mathrm{~ms}$. With target duration of $48 \mathrm{~ms}$ performance improves with decreasing mask duration (main effect mask duration: $F(2,36)=35.55, p<.001$ ) and follows a Type B masking function (main effect SOA: $F(5,90)=49.63, p<.001$ ). However, graphs of different mask durations have distinct time courses (2-way-interaction mask duration $\mathrm{x}$ SOA: $F(10,180)=4.06, p=.005)$. With increasing mask duration the slope of the masking function increases and the minimum of the masking function shifts towards shorter SOAs. The effect of mask duration is most prominent at longer SOAs and the minima are shifted towards longer SOAs.

\section{Correlation of Discrimination Sensitivity With Individual Factor Scores}

It is important to note that in Experiment 1.2 all participants show Type B masking for all mask durations. Thus, several Type A observers from Experiment 1.1 changed their individual masking type from A to B. Nevertheless, masking effects found in Experiment 1.2 are modulated by individual masking types obtained from Experiment 1.1. Figure 3.7 (page 23) shows the correlations of $d^{\prime}$ values correlated with Factor A scores and Factor B scores over all SOAs, for both target durations. The correlations are positive in all conditions, meaning that that a high factor score leads to a high discrimination performance. However, the degree of positive relation varies over the different SOAs depending on the durations of both, target and mask. 

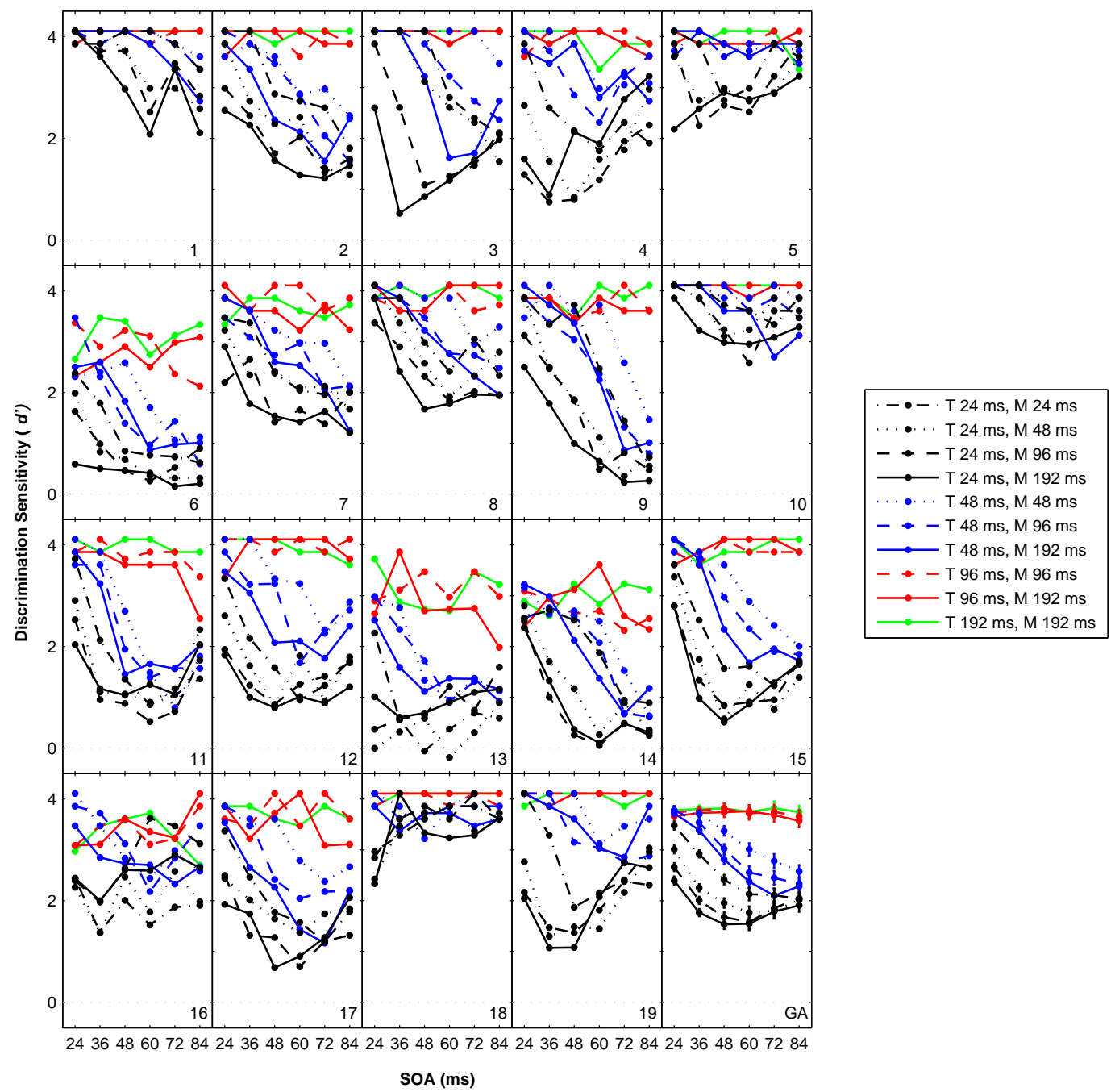

Figure 3.6: Experiment 1.2. Discrimination Sensitivity $d^{\prime}$ (individual plots [1-19] and group average [GA]). In the legend $\mathrm{T}$ refers to target duration and $\mathrm{M}$ refers to mask duration. Error bars represent the within-subject standard error (Loftus \& Masson, 1994).

Target Duration 24 ms. For the shortest SOA the correlation of Factor A scores with $d^{\prime}$ values is near to zero for all mask durations. It increases with increasing SOA (2-way-interaction Factor A x SOA: $F(5,80)=4.64, p<.017)$. The longer the mask duration becomes the stronger becomes the correlation even for intermediate and short SOAs (3-way-interaction Factor A $\mathrm{x}$ SOA x mask duration: $F(15,240)=3.48, p=.011)$. Except for the longest mask duration, the correlation for the intermediate SOAs increases with mask duration. The highest correlation has Factor A in SOAs between $60 \mathrm{~ms}$ and $84 \mathrm{~ms}$.

The correlation of Factor B scores with $d^{\prime}$ values decreases with increasing SOA, but not significantly (2-way-interaction Factor B x SOA: $F(5,80)=1.31, p=.285$ ). The SOA with the strongest correlation shifts to shorter SOAs with increasing mask duration (3-way-interaction 


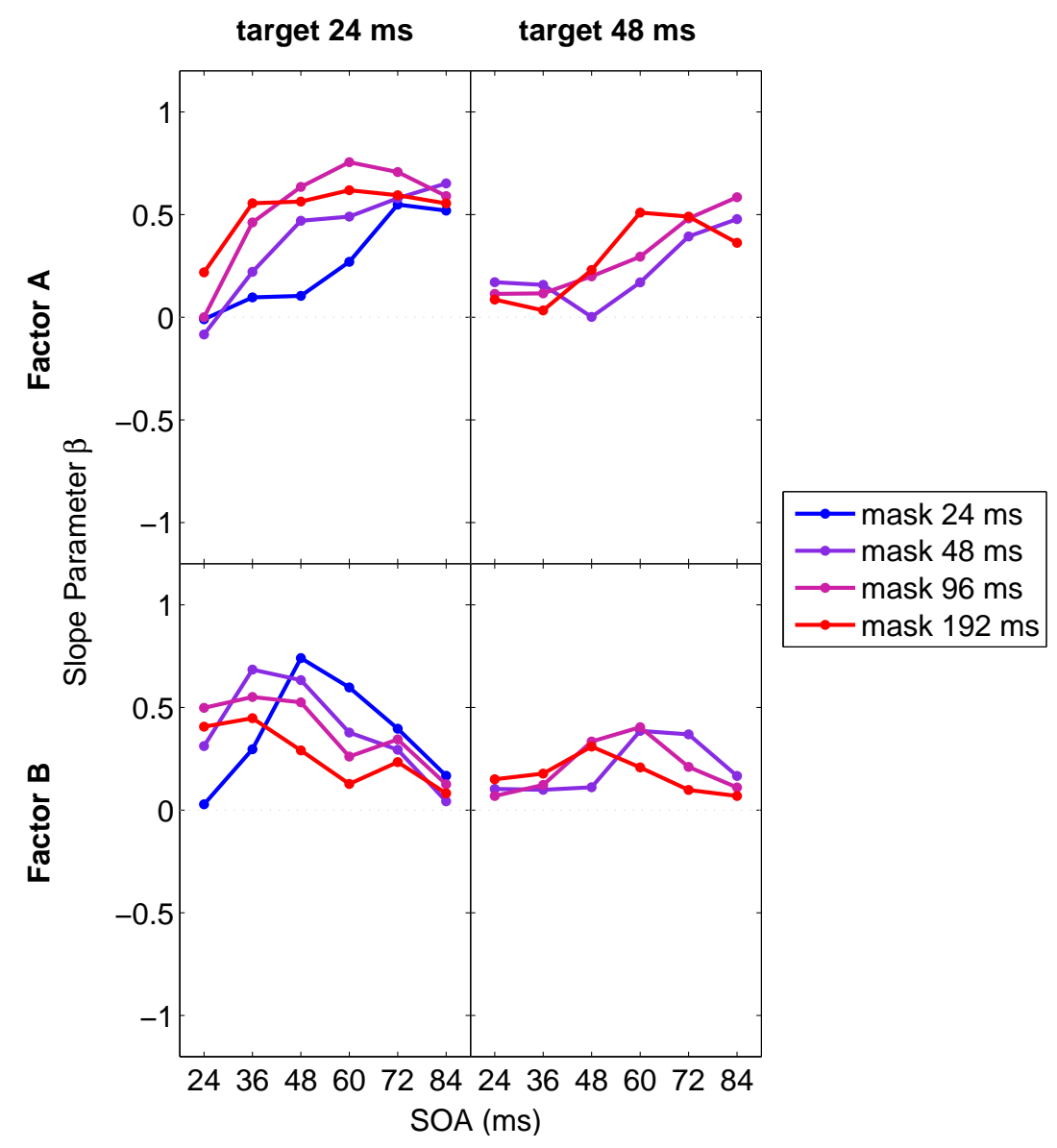

Figure 3.7: Experiment 1.2. Slope parameter $\beta$ over SOAs, with Discrimination Sensitivity $d^{\prime}$ values correlated with Factor A scores (upper row) and Factor B scores (lower row), for target duration $24 \mathrm{~ms}$ (left column) and target duration $48 \mathrm{~ms}$ (right column).

Factor B x SOA x mask duration: $F(15,240)=3.50, p=.011)$ : The correlation of Factor B is highest at SOA $48 \mathrm{~ms}$ when mask duration is $24 \mathrm{~ms}$, but at SOA $36 \mathrm{~ms}$ when mask duration is $48 \mathrm{~ms}$, and at SOA $24 \mathrm{~ms}$ when mask duration is $96 \mathrm{~ms}$ or $192 \mathrm{~ms}$. Until this top point the correlation increases and afterwards it decreases with increasing SOA.

Target Duration 48 ms. In general, correlations are not as strong as for target duration $24 \mathrm{~ms}$. There is nearly no correlation of Factor A scores with $d^{\prime}$ values in short SOAs, but is then increasing with increasing SOA (2-way-interaction Factor A x SOA: $F(5,80)=4.94$, $p=.008)$. Regarding the SOA $60 \mathrm{~ms}$ the correlation of Factor A increases with increasing mask duration, but there is no significant interaction of Factor A with mask duration (2-wayinteraction Factor A x mask duration: $F(2,32)=1.07, p=.336)$.

For the shortest and the longest SOAs there is no correlation of Factor B scores with $d^{\prime}$ values, whereas in intermediate SOAs there is an apparent correlation, but it turned out to be 


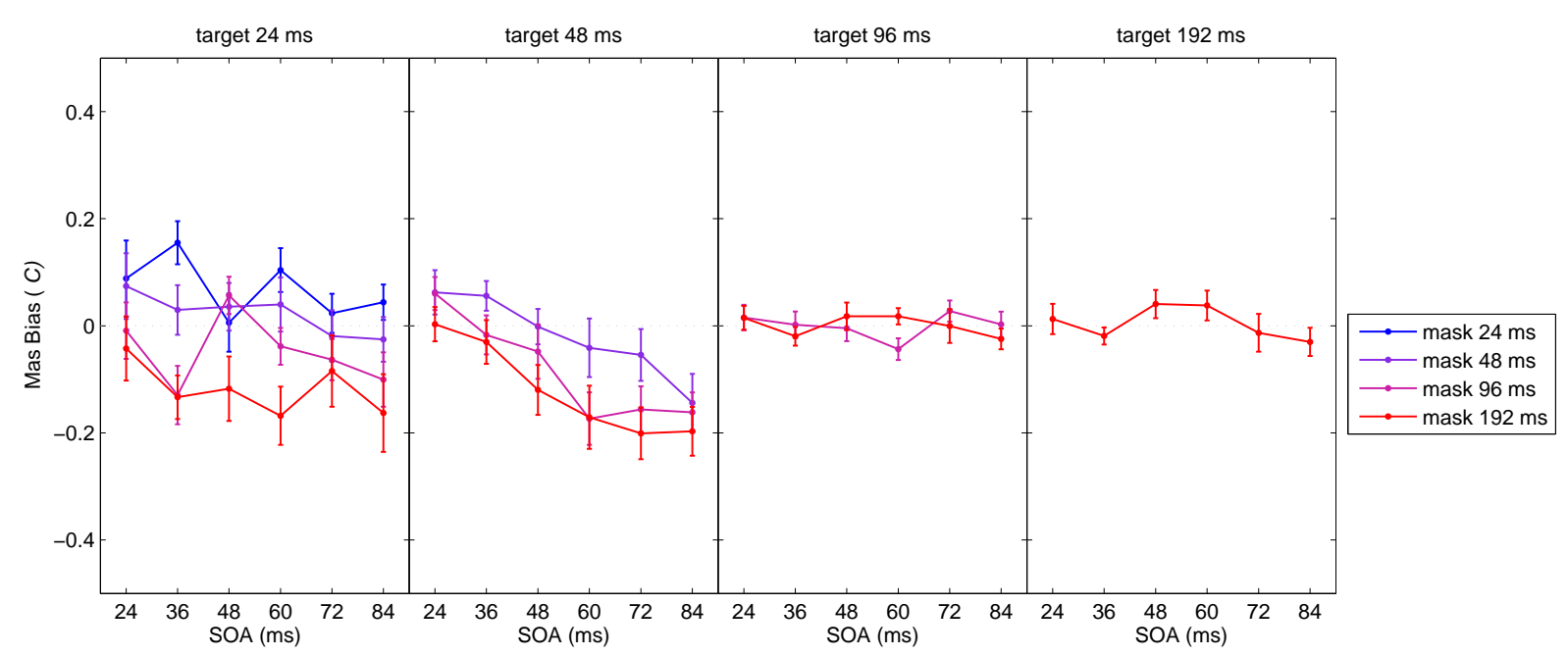

Figure 3.8: Experiment 1.2. Mask Bias $C_{M}$ (group average). Each diagram has one fixed target duration, and all corresponding mask durations are presented by different graphs (see legend). Error bars represent the within-subject standard error (Loftus \& Masson, 1994).

not significant (2-way-interaction Factor B x SOA: $F(5,80)=1.01, p=.388$ ). The SOA with the highest correlation of Factor B with $d^{\prime}$ values increases with decreasing mask duration, but there is no significant interaction with Factor B scores with mask duration (2-way-interaction Factor B x mask duration: $F(2,32)=0.29, p=.670)$.

\section{Mask Bias}

The mean Mask Bias in Experiment 1.2 as function of SOA, target duration and mask duration is shown in Figure 3.8 (page 24). As for Sensitivity, analyses were limited on target durations of $24 \mathrm{~ms}$ and $48 \mathrm{~ms}$. Individual functions with all conditions are shown in Figure 3.9 (page 25).

Target Duration 24 ms. There is a slight bias to respond against the form of the mask when mask duration is $96 \mathrm{~ms}$ or $192 \mathrm{~ms}$, and according to the form of the mask when mask duration is $24 \mathrm{~ms}$ (main effect SOA: $F(5,90)=5.03, p=0.010$ ). Mask Bias depends neither on mask duration (main effect mask duration: $F(3,54)=1.62, p=.195$ ), nor is there a significant interaction between SOA and mask duration $(F(15,270)=1.22, p=.306$, Figure 3.8) (page 24).

Target Duration $48 \mathrm{~ms}$. There is a slight bias responding against the form of the mask in long SOAs (main effect SOA: $F(5,90)=6.55, p=.001$, Figure 3.8) (page 24). Mask Bias depends on mask duration (main effect mask duration: $F(2,36)=4.26, p=.022$ ), but there is no significant interaction of SOA x mask duration $(F(10,180)=0.74, p=.690)$. 


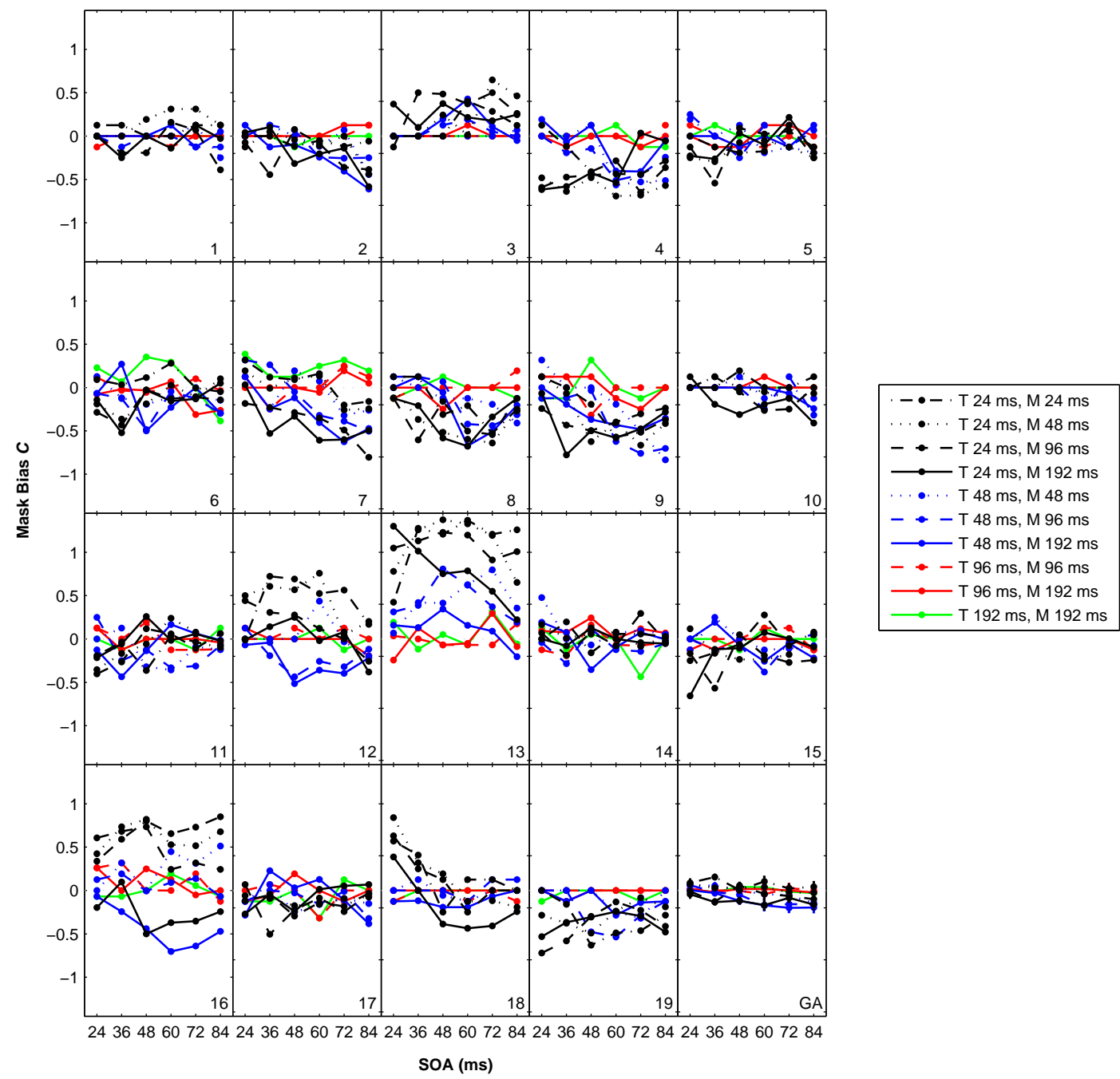

Figure 3.9: Experiment 1.2. Mask Bias $C_{M}$ (individual plots [1-19] and group average [GA]). In the legend $\mathrm{T}$ refers to target duration and $\mathrm{M}$ refers to mask duration. Error bars represent the within-subject standard error (Loftus \& Masson, 1994).

\section{Correlation of Mask Bias With Individual Factor Scores.}

Target Duration 24 ms. See Figure 3.10 (page 26) for the slope parameter over all SOAs, with $C_{M}$ values correlated with Factor A scores and Factor B scores. The slope parameter of Factor A scores with $C_{M}$ values decreases with increasing SOA (2-way-interaction Factor A x SOA: $F(5,80)=4.51, p=.014)$. There is no significant interaction, neither between Factor A scores with mask duration $(F(3,48)=0.56, p=.641)$, nor between Factor B scores and SOA $(F(5,80)=0.82, p=.462)$, nor between Factor B scores and mask duration $(F(3,48)=0.39$, $p=.759)$. 


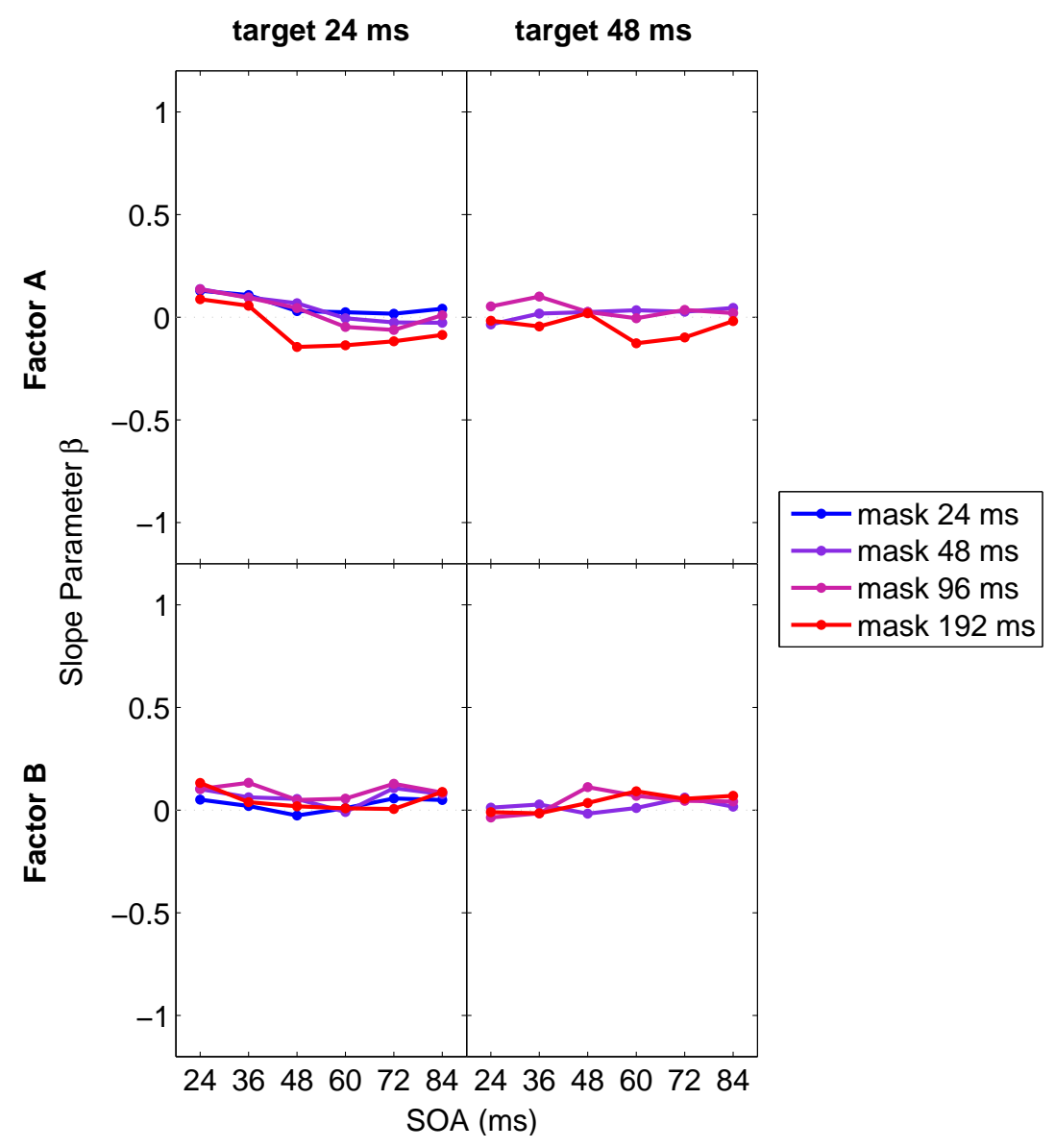

Figure 3.10: Experiment 1.2. Slope parameter $\beta$ over SOAs, with Mask Bias $C_{M}$ values correlated with Factor A (upper row) and Factor B scores (lower row), for target duration $24 \mathrm{~ms}$ (left column) and target duration $48 \mathrm{~ms}$ (right column).

Target Duration 48 ms. See Figure 3.10 (page 26) for the slope parameter over all SOAs, with $C_{M}$ values correlated with Factor A scores and Factor B scores. There are no significant interactions, neither between SOA with Factor A scores $(\mathrm{F}(5,80)=1.85, p=.160)$, nor between SOA with Factor A $(F(5,80)=0.55, p=.623)$, nor Factor B with mask duration $(F(2,32)=1.02$, $p=.370)$, nor Factor B with mask duration $(F(2,32)=0.36, p=.698)$.

\subsubsection{Experiment 1.3}

\section{Discrimination Sensitivity}

In Experiment 1.3 the participants still show diverse masking functions (Figure 3.1 o, page 17), however two facts are apparent. First, all masking functions seem to become more Type-B-like. Participants who showed a Type B masking function in the first part strengthened their type. Furthermore, participants who showed a Type A masking function in the first part (e.g. num- 
(a)

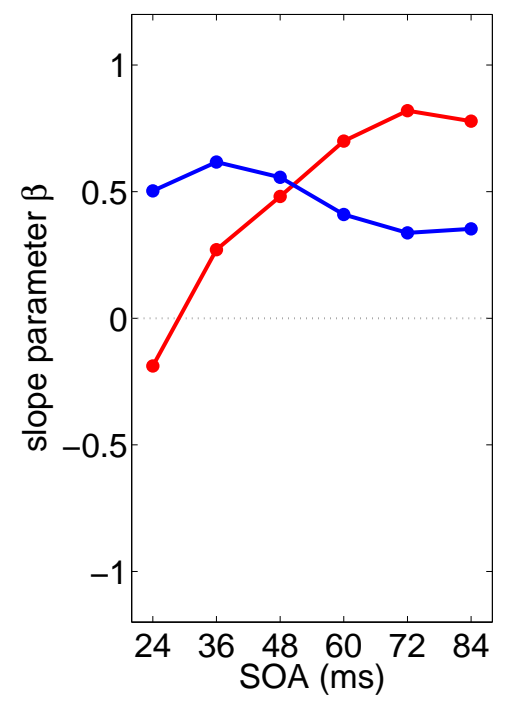

(b)

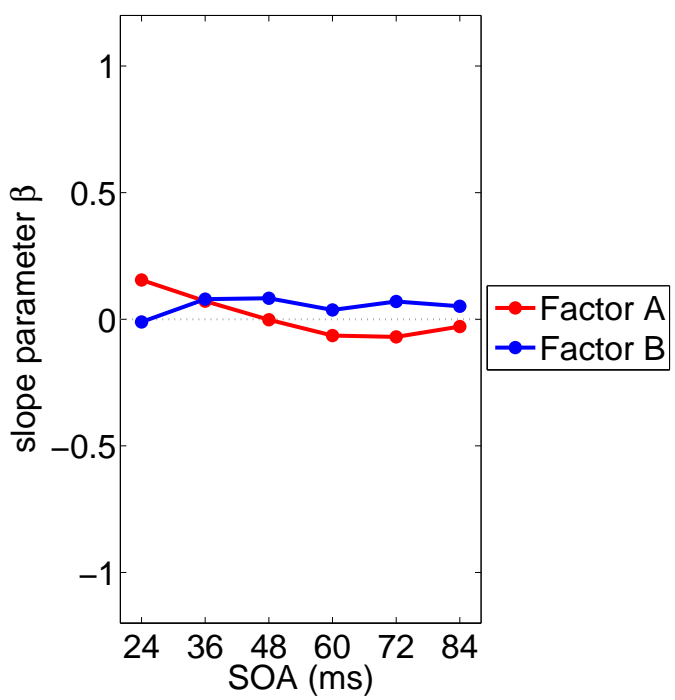

Figure 3.11: Experiment 1.3. Slope parameter $\beta$ over SOAs, with (a) Discrimination Sensitivity $d^{\prime}$ and (b) Mask Bias $C_{M}$ values correlated with Factor A scores and Factor B scores.

ber 5 or 12), now show a Type B masking function. Second, the general performance level is increased in almost all participants. The group average resembles a Type B function (with a clear negative slope, whereas in Experiment 1.1 there was no slope). The ANOVA confirms these observations: Sensitivity differs across Experiment 1.1 and 1.3 (two-way interaction Experiment x SOA: $F(5,90)=7.51, p=.002)$.

Correlation of Discrimination Sensitivity With Individual Factor Scores. The correlation of Factor A scores with $d^{\prime}$ values increases with increasing SOA (2-way interaction Factor A scores x SOA: $F(5,80)=9.15, p<.001$, Figure 3.11a, page 27). The correlation of Factor B scores with $d^{\prime}$ values is positive for all SOAs and is only slightly smaller in long SOAs than in short SOA (2-way interaction Factor B scores x SOA: $F(5,80)=0.79, p=.505$, Figure 3.11a, page 27).

\section{Mask Bias}

In most of the datasets $C_{M}$ values are reduced in comparison to Experiment 1.1 becoming negative or approach zero (Figure 3.2, page 18, o). The group average shows no bias to respond either according or contrary to the shape of the mask stimulus. The Mask Bias differs across Experiment 1.1 and 1.3 significantly (two-way interaction Experiment x SOA: $F(5,90)=6.93$, $p=.001)$. 


\section{Study 1 - Effect of the Ratio of Target Duration to Mask Duration}

Correlation of Mask Bias With Individual Factor Scores. The correlation of Factor A scores with $C_{M}$ values is slightly positive in short SOAs and slightly negative in long SOAs. Although these variations appear minimal, these variations across SOAs became significant (2way interaction Factor A x SOA: $F(5,80)=3.55, p=.024$, Figure 3.11b, page 27). In contrast, the correlation of Factor B scores with $C_{M}$ values, being slightly positive across all SOAs except the shortest, does not differ significantly across SOAs (2-way interaction Factor B scores x SOA: $F(5,80)=0.57, p=.628$, Figure $3.11 \mathrm{~b}$, page 27$)$. There was no main effect of Factor B scores neither $(F(1,16)=0.34, p=.566)$.

\subsection{Discussion}

\subsubsection{Experiment 1.1}

This experiment aimed to obtain inter-individual differences in the Discrimination Sensitivity using them for the factor analysis, whose factors represent potential processes involved metacontrast masking. Earlier findings of substantial inter-individual differences in metacontrast masking were replicated, with some participants exhibiting increasing masking functions and some participants exhibiting decreasing masking functions (Albrecht \& Mattler, 2012a, 2012b). This data pattern can be described well by two underlying latent variables explaining approximately $93 \%$ of total variability: The first latent variable reflects an increasing masking function, the second one reflects a decreasing masking function. This finding corroborates an earlier study, in which a very similar factor structure was found where two factors explained $88 \%$ of total variability (Albrecht \& Mattler, 2016).

These two factors may be regarded as reflecting two independent (perceptual) processes that jointly determine the shape of the masking function. The impact of each process depends on SOA and the specific weight of each participant. Under this assumption individual factor scores, obtained by regression, can be regarded as estimates of individual weights of each underlying variable. Depending on the score of each factor Type A, Type B, or mixed type functions occur. The higher the factor score for Factor A and the lower the factor score for Factor B the more prominent is a Type A function, and consequently, the higher the factor score for Factor B and the lower the factor score for Factor A the more prominent is a Type B function.

Type A observers typically have a strong bias to respond according to the shape of the mask, especially in short SOAs, as here the perception of apparent motions are weak. With increasing SOA this bias decreases. By contrast, Type B observers typically have as slight bias to respond contrary to the shape of the mask, equally over all SOAs. So, in short SOAS there is high variability while in in long SOAs there is not. In short SOAs there is a strong positive correlation between $C_{M}$ values and Factor A scores indicating that a high Factor A score predicts a high 
positive Mask Bias. The correlation between $C_{M}$ values and Factor B scores is negative in short SOAs, indicating that a high Factor B score predicts a negative Mask Bias. In long SOAs both Factors show no correlation between factor scores and Mask Bias indicating that the height of the Factor A or B score does not reliable predict the Mask Bias in long SOAs.

Type A and Type B observers have different criterion contents when fulfilling the task. More precisely, Type A observers report to see and use percepts of apparent motion, a rotating movement in incongruent trials and an expanding movement in congruent trials, while Type B observers report to see and use percepts of negative afterimages as a conjoint percept of target and mask. As different criterion contents result in different masking functions (Breitmeyer \& Ö $\breve{g}$ men, 2006) they are useful information, which can be used to infer the underlying processes of Factor A and B. The process underlying Factor A is strongly associated with a Type A masking function, which in turn go along with a percept of motion.

According to the Integration-Segregation-Approach by Neumann (1978) and Reeves (1982) the perception of two stimuli being segregated, enabling apparent moving images are associated to the segregation process. Thus, the Factor A process may correspond to the segregation process. Furthermore, the process represented by Factor B is strongly associated with a Type B masking function, which in turn go along with a percept of negative afterimages inside of the mask stimulus. The percept of a simultaneous, conjoint image of two physically succeeding stimuli is associated to the integration process of the same model and thus, the Factor B process may correspond to the integration process.

\subsubsection{Experiment 1.2}

This experiment examined how stimulus presentation durations affect individual masking functions to determine the characteristics of the processes involved in metacontrast masking. By modulating the target's and mask's presentation durations masking functions change. When both, target and mask are presented for $24 \mathrm{~ms}$ (T/M ratio 1), the masking function is steadily decreasing with a minimum at a SOA of $84 \mathrm{~ms}$. When the mask is presented for $192 \mathrm{~ms}$ (T/M ratio 0.125 ), the first half of the masking function decreases to a minimum at $48 \mathrm{~ms} \mathrm{SOA}$, the second half increases again. Mask durations of $48 \mathrm{~ms}$ and $92 \mathrm{~ms}$ have intermediate T/M ratios and produce masking functions fitting gradually between those of the shortest and longest duration. When the target is presented for $48 \mathrm{~ms}$ and the mask for $192 \mathrm{~ms}$ (T/M ratio 0.25), the minimum of the masking function is at the SOA of $72 \mathrm{~ms}$. The shorter the mask is presented (the higher the $\mathrm{T} / \mathrm{M}$ ratio), the less deep is the slope of the monotonically decreasing masking function with minima at $84 \mathrm{~ms} \mathrm{SOA} \mathrm{(limit} \mathrm{of} \mathrm{measurement).}$ 


\section{Study 1 - Effect of the Ratio of Target Duration to Mask Duration}

Summarizing these results, the longer the mask duration becomes in relation to the target duration (the smaller the T/M ratio), the more shifts the minimum of the masking function towards shorter SOAs, and so the more Type-A-like (monotonically increasing) the function becomes. This replicates findings described by Weisstein (1972) and Breitmeyer (1978) and confirms the stimuli used here. In addition, results for more comprehensive variation of target's and mask's durations for varying SOAs were introduced.

Overall, the sensitivity is higher in all conditions when the target is presented for $48 \mathrm{~ms}$ than when it is presented for $24 \mathrm{~ms}$, even for equal T/M ratio values. Additionally, the masking functions seem to be shifted towards longer SOAs the longer the target duration becomes. This implicates that the shift towards shorter SOAs with decreasing T/M ratio holds only true for fixed target durations. Therefore, the course of the masking function is determined by an interaction of $\mathrm{T} / \mathrm{M}$ ratio and target duration.

As stated in Experiment 1.1, individual masking functions can be described by two neural processes, represented by Factor A and Factor B, and having distinct influences in different conditions. Process A's impact increases with increasing SOA. While in long SOAs the impact is independent of target and mask durations, the T/M ratio plays a role in short and intermediate SOAs: The more favorable conditions are for a Type A function (the longer the mask/ the smaller the T/M ratio), the more impact has Process A even in short SOAs. Process B has its strongest impact in short and medium-length SOAs. The more favorable conditions are for a Type B function (the shorter the mask/ the higher the T/M ratio) the more shifts the maximum impact of Process B from short to intermediate SOAs. In the target duration of $48 \mathrm{~ms}$ both processes show a similar pattern like in target duration of $24 \mathrm{~ms}$, but the influence is weaker and effects are not statistically significant. Like in the sensitivity data this pattern is slightly shifted towards longer SOAs.

These results implicate that both processes are most prominent in SOAs related to best sensitivity in masking functions (Type A in long SOAs and Type B in short SOAs), independent of mask durations. In addition, when mask durations are considered, both processes have their strongest impact in conditions being favorable for the corresponding functions, and when these conditions are less favorable, the impact broadens to adjacent SOAs. When the target is presented for $48 \mathrm{~ms}$, both processes have no impact on the pronouncement of the sensitivity. This coherence can be explained by the fact that in these SOAs the sensitivity is very high, not needing a strong process to regulate the performance. In intermediate SOAs both processes have equal impact and in long SOAs Process A prevails.

Combining the Integration-Segregation-Theory by Neumann (1978) and results of the present study, following statements may be inferred: The segregation process represented by Process A has its strongest influence in long SOAs and this influence weakens the shorter the SOA be- 
comes. In contrast, the integration process represented by Process B has its strongest influence in very short SOAs and this influence weakens the longer the SOA becomes. Therefore, for intermediate SOAs there might be a competition between those two processes, shifting the maximal masking effect along shorter or longer SOAs resulting in a more J-like (or even Type A) course or a U-like (Type B) course. The maximal masking effect may be caused by either an equilibrium (where no process prevails and therefore percepts are ineffectual) or an inactivity of both processes (where stimuli are insufficiently processed for effectual percepts). In this experiment, segregation occurs in long SOAs, and with increasing mask duration, stimuli were increasingly segregated even in short SOAs. Therefore, it may be assumed that a higher mask energy suppresses the integration process of target and mask and favors the segregated perception for intermediate SOAs, shifting the maximal masking to lower SOAs. Integration occurs in short and medium-length SOAs, and with decreasing mask duration this coherence is shifting to intermediate SOAs. Therefore, it may be assumed that a high T/M ratio supports the integration process for intermediate SOAs shifting the maximal masking to longer SOAs. With longer target durations the integration process is even stronger extending even over long SOAs shifting the maximal masking to even longer SOAs.

All participants show a Type B masking functions even when they had high loads for Factor A. This may be explained with the longer presentation durations of the target stimulus in the whole experiment including target presentation durations of $96 \mathrm{~ms}$ and $192 \mathrm{~ms}$ as well, where there was no masking effect any more. With longer stimulus durations there may be longer time for stimulus integration shifting the maximal masking to intermediate SOAs effecting a Type B course. In the context of the experiment the integration process may be more activated prevailing the segregation process and therefore forms the masking function. To confirm this assumption a comparable experiment has to be conducted without those two long target presentation durations. When all participants still show a Type B masking function there has to be an alternative explanation.

The Mask Bias is stable across all conditions. In contrast to Experiment 1.1 the processes represented by Factor A and Factor B seem to have no impact on its manifestation neither in the short SOAs. So the height of the Factor A and B scores does not predict the Bias.

\subsubsection{Experiment 1.3}

The training during the second experiment influences the masking functions of the third experiment. This results in a change of the masking type in some participants and in a general performance level increase. Both processes represented by Factor A and B still have an impact on the masking function - the higher the factor scores, the higher the sensitivity. This impact increases with increasing SOA for Factor A scores. For Factor B scores it is equally high and 
positive over all SOAs. This is remarkable as in Experiment 1.1 the impact was negative in short SOAs and nonexistent in long SOAs. Thus, the segregation process leads to a performance increase in long SOAs, the integration process leads to a general performance increase. Mask Bias has changed in comparison to Experiment 1.1, which is in contradiction to Schwiedrzik, Singer, and Melloni (2009) who found no change in the bias over training. As both processes are unrelated to the Mask Bias, individual factor scores seem to have no predictive value any more. Compared to earlier studies (Albrecht et al., 2010), in which participants hardly changed their masking functions over time or situations, masking functions in the present study shifted to Type B. This finding suggests that Type A observers adapt their identification strategies to the specific conditions and that they keep this change. A well pronounced B process may support this maintained changed. It may be that the integration process was trained in Experiment 1.2, enabling a facilitating activation, with the result that it can now prevail over the segregation process.

\subsection{Conclusion}

In this study inter-individual differences in metacontrast masking and their interaction with varied stimulus durations were investigated. There are three major findings: First, earlier findings were replicated. Inter-individual differences in metacontrast masking can be described by a twofactor-structure, which reflects Type A and Type B masking, respectively (Albrecht \& Mattler, 2016). These two masking types can be conceptualized as two different processes determining the masking functions. Taking the Integration-Segregation-Theory by Neumann (1978) and Reeves (1982) as basis, the process underlying a Type A masking function, where sensitivity increases with SOA, can be described with a segregation process. Perceiving stimuli successively enables the perception of apparent motion. The process underlying a Type B masking function, where sensitivity decreases with SOA, can be described with the integration process.

Second, earlier findings that the target-to-mask ratio influences the type of the masking functions were replicated and even extended by the variation of target duration, mask duration, and SOA. The segregation process has its major impact in long SOAs, independent from stimulus durations. But it is also effective in short SOAs when the T/M ratio is low (long mask duration in relation to target duration). The integration process has its major impact in short and medium length SOAs, dependent on mask duration. It is most effective, when the $\mathrm{T} / \mathrm{M}$ ratio is high (target and mask duration are equal or the difference is low).

Third, a training effect was found, as there is a general sensitivity increase from Experiment 1.1 to Experiment 1.3. Additionally, a context effect was found as all participants show a Type B masking function in Experiment 1.3 although some of them showed a Type A masking 
function in Experiment 1.1. It is hypothesized that this change is due to context effects in Experiment 1.2 , where many conditions favored a Type B masking and so the perceptual cue got salient. 



\section{Study 2 - Effect of Stimulus Size and Eccentricity}

Visual processing in the peripheral visual field is different from visual processing in the fovea. This is due to anatomical differences in the retina and the subsequent processing paths in the cortical areas of the brain. In the peripheral parts of the retina the receptive fields are larger with more M-ganglion cells than P-ganglion cells and the proportion of rods over cones is higher than in the fovea (Lee, Martin, \& Grunert, 2010). All these factors lead to a higher temporal resolution favoring motion detection and a lower spatial resolution of vision impairing contour detection. Many psychological studies investigated over centuries the influence of peripheral presented stimuli on perception and performance in different tasks, using (among others) the metacontrast masking paradigm.

Behavioral performance of some visual tasks decreases with eccentricity while others increase: The effect of metacontrast masking is larger in the periphery than in the fovea and therefore the target detection performance is decreasing with increasing eccentricity (Francis, 2003). As spatial resolution decreases with increasing eccentricity it is interesting to examine the effects of different stimulus sizes in relation to different retinal positions. In most of the studies the size of the mask was varied while the size of the target was held constant. Matteson (1969) used a disc as target with a size of $0.5^{\circ}$, and an annulus with a diameter of either $0.625^{\circ}$, 1.5 , or $2.5^{\circ}$ was used as mask. Stimuli were presented peripherally, the target $1.5^{\circ}$ below a fixation point and the mask $1.5^{\circ}$ above of the fixation point. He found that the masking effect increased with increasing size of the mask. Schiller and Greenfield (1969) used disk-shaped light flashes and varied the size of the mask flash between $0.29^{\circ}$ and $1.02^{\circ}$ visual angle, while keeping the target constant at $0.25^{\circ}$. They found that masking functions over ISI shift towards Type A with increasing mask size, and towards Type B masking function when the size of target and mask are more similar. In contrast, Duangudom, Francis, and Herzog (2007) used vernier stimuli and by manipulating their number or length, the strength of masking and the shape of the masking function is altered. The more flanking mask stimuli were presented the more the masking function shifts from Type B to Type A. With one flanking mask masking becomes stronger the longer the masks are, having always a Type B course. With two flanking masks the masking function shifts from Type B to Type A when the length of the mask stimuli are 


\section{Study 2 - Effect of Stimulus Size and Eccentricity}

increased. Bridgeman and Leff (1979) used disc and annulus stimuli and varied the size of both stimuli, and in addition compared the masking effect when stimuli were presented foveally with when they were presented peripherally. Target and mask stimuli had equal area, the target diameter varied between $0.25^{\circ}, 0.73^{\circ}$, and $1.8^{\circ}$, the mask outside diameter varied between $0.38^{\circ}$, $1.07^{\circ}$, and $2.63^{\circ}$ of visual angle. The SOA was varied between $0,40,80$, and $120 \mathrm{~ms}$ and the eccentricity was varied between $0^{\circ}$ (foveal), $4.6^{\circ}$, and $9.2^{\circ}$ visual angle. In general, masking is stronger in small stimuli than in large stimuli, and masking is stronger in the periphery than in the fovea. Furthermore stimulus size and eccentricity have an interacting influence on the masking function: masking is strongest when small stimuli were presented foveally, and when large stimuli were presented in the periphery. In the periphery masking is stronger for large stimuli than for small stimuli. The same interaction found Johnson, Keltner, and Balestrery (1978) as well, namely in the detection and resolution threshold sensitivities, where the effect of target size is greater in the periphery. They used circle and square outlines as targets and varied the size of the circle between $0.15^{\circ}$ and $1.1^{\circ}$ of visual angle. Also Sturr, Frumkes, and Veneruso (1965) investigated different stimulus sizes and presentation positions. They used circles for both stimuli, the target size was kept constant at $0.17^{\circ}$ and the mask was varied between $0.25^{\circ}$ and $0.50^{\circ}$. Stimuli were presented either foveally or in the periphery at $2^{\circ}, 5^{\circ}$, or $7^{\circ}$ on the left visual field. The results are comparable. Masking was stronger with smaller masking stimuli than for larger stimuli in all positions, and stronger in the periphery than in the fovea for all sizes.

This study aims to determine how the processes involved in metacontrast masking behave when stimulus presentation position and size are manipulated. Here, inter-individual differences were used to get information about the characteristics of the processes. The study is composed of three experiments: In Experiment 2.1 the SOA is varied in a metacontrast paradigm. It is expected to obtain individually different masking functions of each participant, which can be used for the factor analysis, whose factors describe the individual differences and represent the underlying processes. In Experiment 2.2 the presentation position and the size of the stimuli is varied in addition to the SOA. Stimuli are presented either peripherally or foveally either small or large. The size of the target as well as the mask stimulus are adapted, keeping the relation constant. Due to the proportional scaling of both stimuli, the effect of the general stimulus size in different retinal positions were examined and not the effect of mask size in relation to target size. Furthermore, the individual factor scores of the factor analysis of Experiment 2.1 were correlated with the Discrimination Sensitivity and the Mask Bias of Experiment 2.2 to obtain a relation measure, which provide information about the contribution of the specific process on the individual masking function. In Experiment 2.3 individual masking functions of the Experiment 2.1 are compared to those during the Experiment 2.2 with the same conditions to investigate possible training or context effects. 


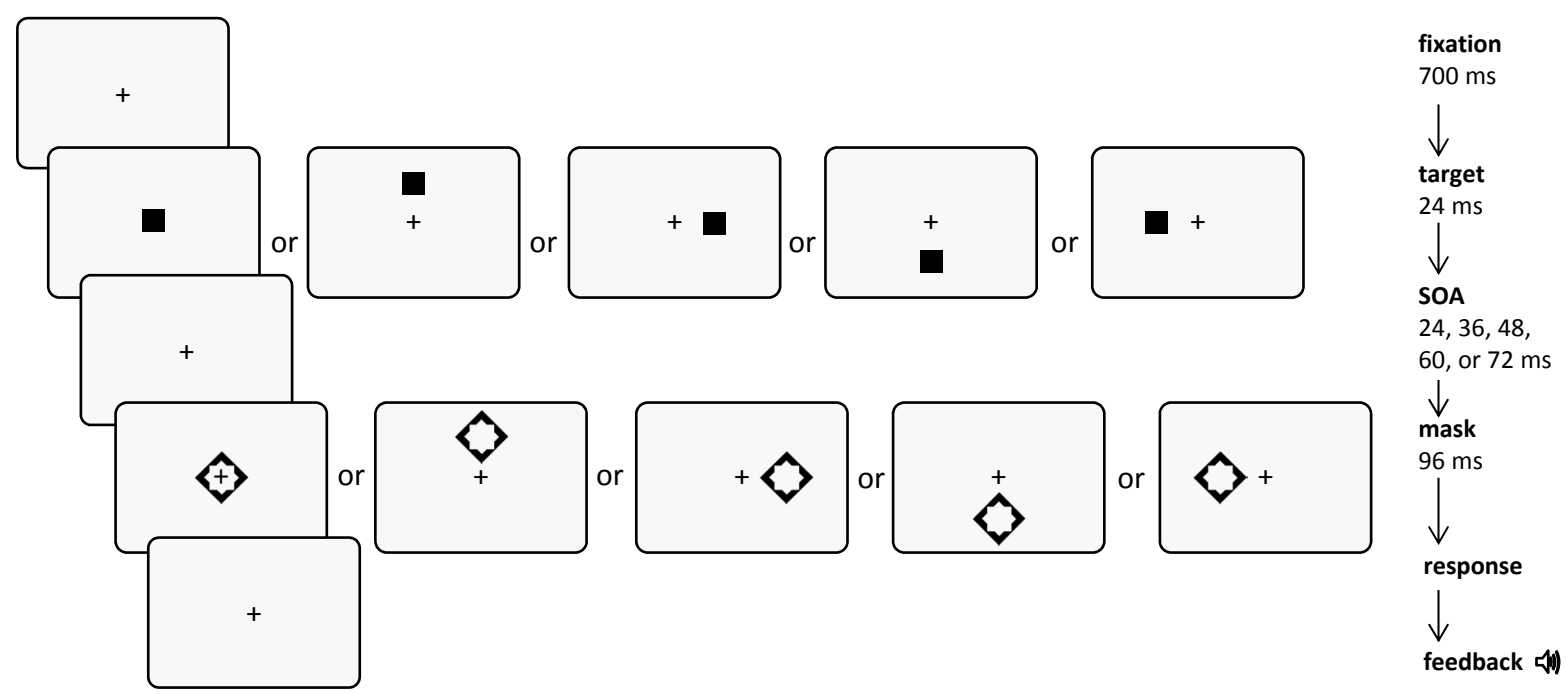

Figure 4.1: Experiment 2.2 and 3.2. Trial course.

\subsection{Methods}

\subsubsection{Participants}

Twenty healthy participants (10 male) participated in this experiment. All but one were righthanded, and their mean age was $\mathrm{M}=24.4$ ( $\mathrm{SD}=3.9$ ), ranging from 19 to 33 .

\subsubsection{Experimental Set-Up}

The study is composed of two Experiments with five sessions in total. In all sessions they had to fulfill the target identification task. Experiment 2.1 includes the first session with 720 trials in 12 blocks (144 repetitions per SOA). Experiment 2.3 includes the first session and specific blocks of sessions two to five with the same conditions like in the first session and the same trial number. They were presented as shown in Figure $2.1 \mathrm{~b}$ (page 10) with the exception that SOA was only varied between $24 \mathrm{~ms}$ and $72 \mathrm{~ms}$.

Experiment 2.2 includes sessions two to five except the blocks with the conditions of Experiment 2.1 with 2400 trials in total. The stimulus size and the eccentricity of the presentation positions were varied. Stimuli were either small with visual angles of $0.5^{\circ}$ for the target and $0.8^{\circ}$ for the mask, or large with visual angles of $3.0^{\circ}$ for the target and $4.8^{\circ}$ for the mask. The presentation position could be either central $\left(0^{\circ}\right.$ eccentricity) or above, below, left to, or right to the center with $5^{\circ}$ eccentricity (see Figure 4.1, page 37 ). Stimulus size and presentation position were presented blockwise and were announced to the participants at the beginning of each block. The SOA was varied between $24 \mathrm{~ms}$ and $72 \mathrm{~ms}$. Per SOA and stimulus size 240 trials were presented. 


\section{Study 2 - Effect of Stimulus Size and Eccentricity}

To control that participants fixate the fixation cross in the center of the display and in this way observe stimuli in the periphery out of focus (for the conditions in the eccentricity), the Eyelink 1000 eyetracker (SR-Research, binocular mode, $1000 \mathrm{~Hz}$ temporal resolution) was used to monitor eye positions and movements. The eye tracking was calibrated at the beginning of the experiment and after breaks on demand. Each trial started not before participants fixate the fixation cross. Viewing distance was $67 \mathrm{~cm}$. To keep the visual angle constant the absolute size of the stimuli in Experiment 2.1 and 2.3 were reduced (in relation to the absolute size with $100 \mathrm{~cm}$ viewing distance).

\subsubsection{Data Analysis}

For all Experiments the Discrimination Sensitivity $d^{\prime}$ and the Mask Bias $C_{M}$ were calculated using the signal detection theory by Macmillan and Creelman (2005) as described in Chapter 2.4. With the data of Experiment 2.1 a factor analysis was conducted as described in Chapter 2.4. Data of Experiment 2.2 were averaged over sessions for each condition. Statistical analyses were run with presentation positions and stimulus sizes as independent variables. In a first step, analyses of variances (ANOVA) were conducted to examine the overall effects of presentation positions and stimulus sizes. In a second step, to test for the effect of individual differences, a full factorial repeated measures analysis of covariance (ANCOVA) was conducted with the within-subject factors SOA and presentation positions and stimulus sizes, and the two continuous between-subject covariates Type A Score and Type B Score obtained in Experiment 2.1. ANCOVA models included all pure within-subject effects, the main effect for each covariate, and the interactions of each covariate with all within-subject effects. Thus, all effects including an interaction of both covariates were excluded. The slope of the regression of $d^{\prime}$ and $C_{M}$ values with the factor scores, the slope parameter $\beta$, is calculated for each SOA, representing the correlation between $d^{\prime}$ and $C_{M}$ values with the factor scores. Statistical analysis was conducted using R (R Core Team (version 3.0.1) [Computer software], 2013).

\subsection{Results}

\subsubsection{Experiment 2.1}

\section{Discrimination Sensitivity and Mask Bias}

The masking functions of each individual participant are shown in Figure 4.2 (•, page 39). Comparable to Study 1 there is substantial inter-individual variability with regard to the slope and the level of masking functions. Some participants show a clear Type A masking function (e.g. number 5, 9, or 11), while other participants show a clear Type B masking function (e.g. number 1, 


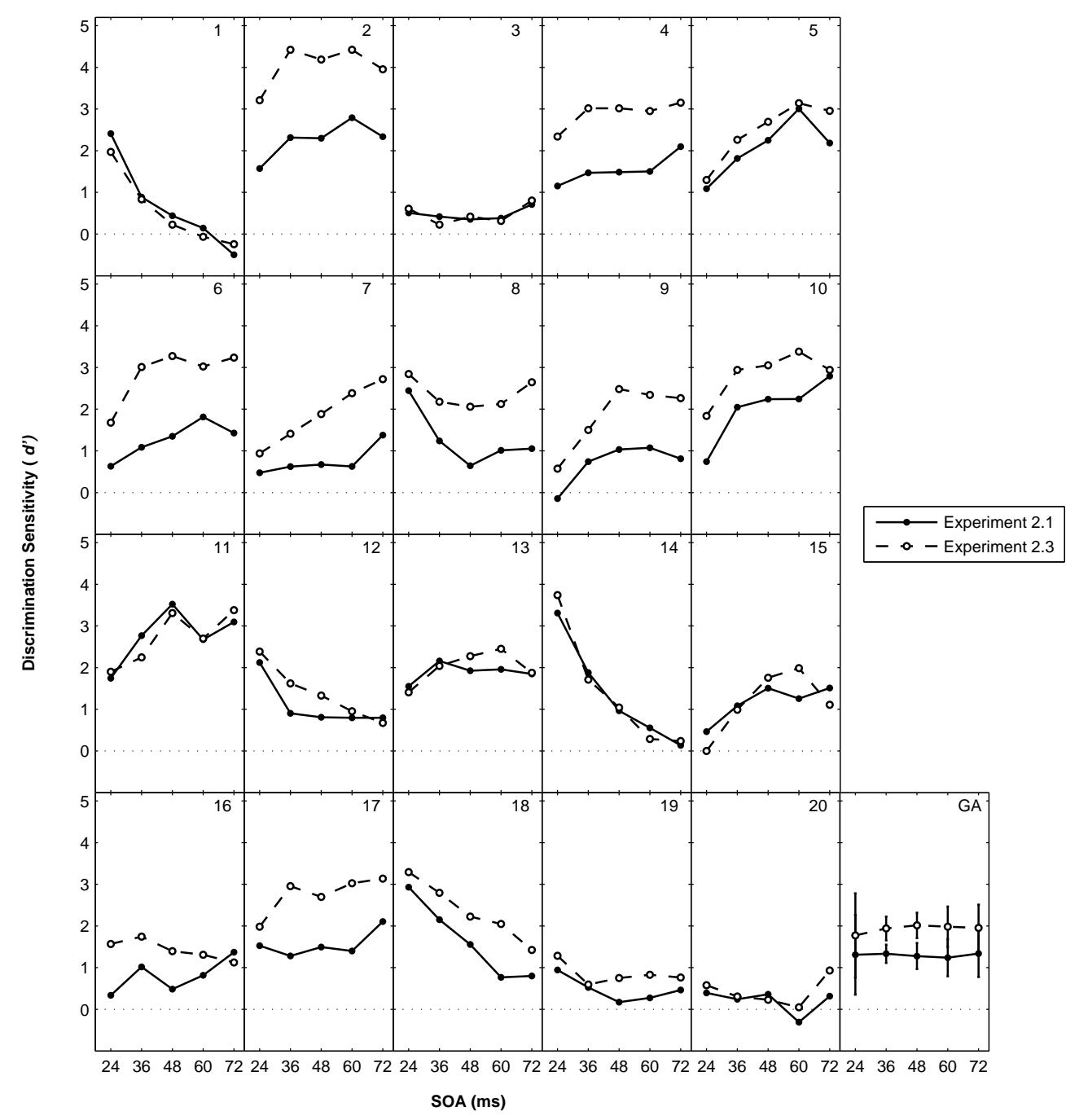

Figure 4.2: Experiment $2.1(\bullet)$ and 2.3. (०). Discrimination Sensitivity $d^{\prime}$ (individual plots [1-20] and grand average [GA]). Error bars represent the within-subject standard error (Loftus \& Masson, 1994).

14, or 18). In addition, the general performance level differs among participants, for example comparing number 3 with number 11 . The average over all datasets results in an inconclusive masking function without variability over SOAs. Accordingly, an one-way repeated-measures ANOVA showed no significant effect of SOA on Sensitivity $d^{\prime}(F(4,76)=0.08, p=.987)$.

Individual data for Mask Bias $C_{M}$ as function of SOA are shown in Figure 4.3 (•, page 40). Positive $C_{M}$ values represent the bias to respond according to the shape of the mask and negative values represent the bias to respond contrary to the shape of the mask. The Mask Bias of the grand average is slightly positive in short SOAs, decreases with increasing SOA and ends slightly negative in long SOAs. This effect proved significant in a one-way repeated-measures ANOVA $(F(4,76)=27.69, p<.001)$. So, Mask Bias differs over different SOAs, in short SOAs 


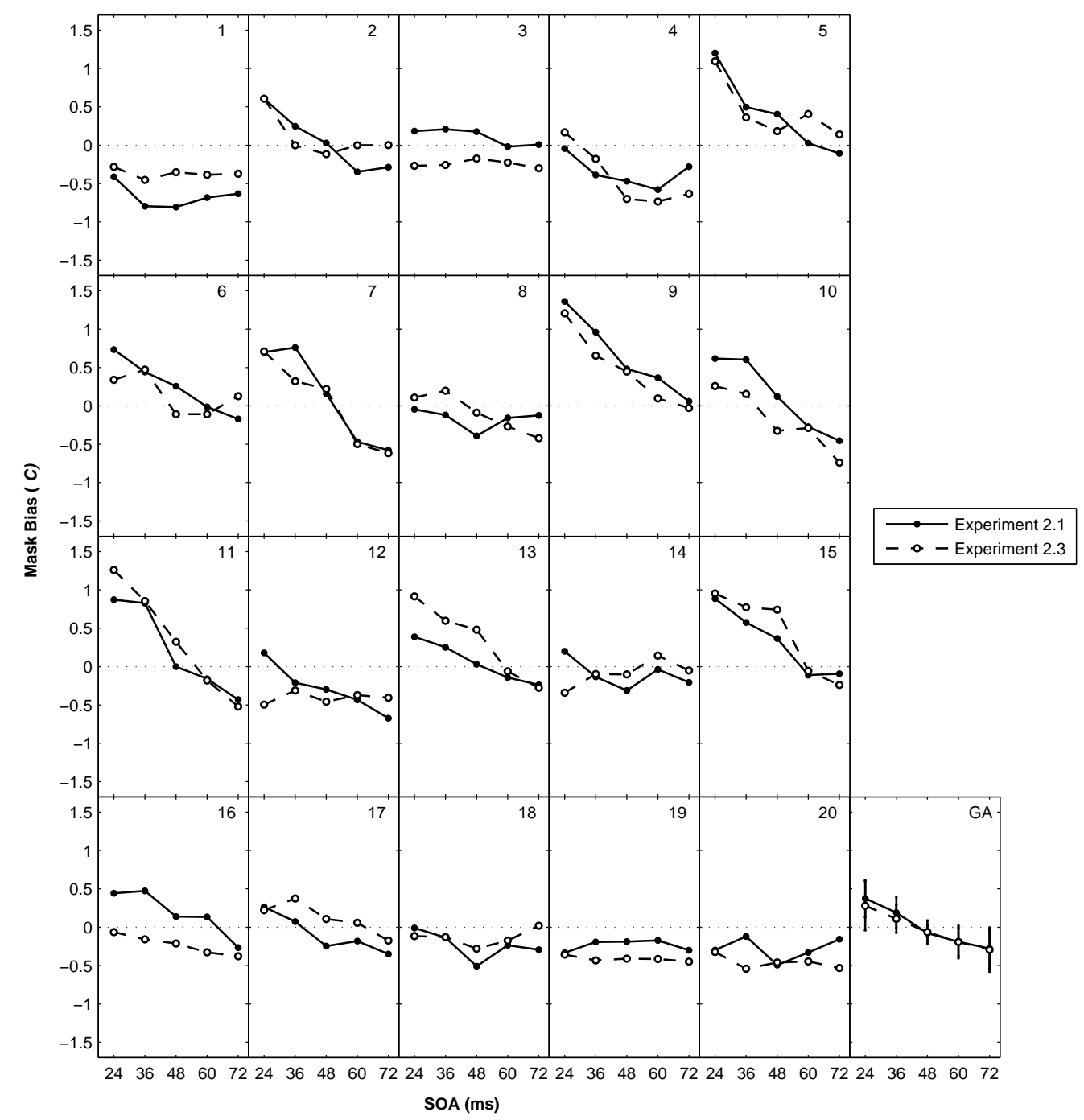

Figure 4.3: Experiment 2.1 (•) and 2.3. (०). Mask Bias $C_{M}$ (individual plots [1-20] and grand average [GA]). Error bars represent the within-subject standard error (Loftus \& Masson, 1994).

the response is made biased according to the mask and in long SOAs the response is made biased contrary to the mask. Having a look on individual data, the Mask Bias can be categorized in two main groups. The first group has an decreasing Bias with increasing SOA, meaning that in short SOAs these participants rely heavily on the shape of the mask. Comparing the Bias to the Sensitivity, these participants show a Type A masking function (e.g. number 5, 9, or 11). The second group has a mostly uniform slightly negative Mask Bias over all SOAs, meaning that the shape of the mask has almost no significance. Comparing the Bias to the Sensitivity, these participants show a Type B masking function (e.g. number 1, 14, or 18). 


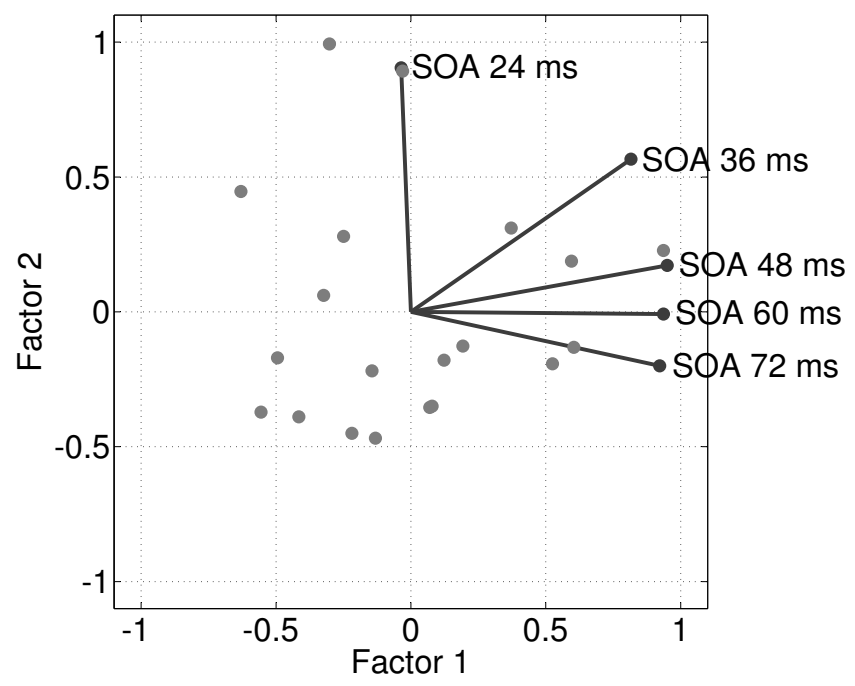

Figure 4.4: Experiment 2.1. Factor analysis - biplot with factor loadings (-) for each SOA and factor scores for each participant $(\bullet)$. (Please note that scales refer to factor loadings.)

\section{Factor Analysis}

The maximum likelihood factor analysis revealed two factors with eigenvalues $>1$, which together explain $90 \%$ of total variance $\left(\chi^{2}(1)=0.21, p=.64\right.$, Table 4.1 , page 42$)$. In contrast, the one-factor solution only explains $66 \%$ of total variability, and thus fits the data only poorly $\left(\chi^{2}(5)=27.3, p<.001\right)$. As only five SOAs were examined a three-factor-analysis is not possible. Therefore, two factors are most appropriate for the data. Loadings of Factor 1 are increasing over SOAs, while loadings of Factor 2 are decreasing (Table 4.1, page 42, and Figure 4.4, page 41). The results are in line with Study 1. As Factor 1 loads high at long SOAs but low at short SOAs, it describes the process leading to a Type A masking function and therefore will be named "Factor A" subsequently. By contrast, Factor 2 loads low at long SOAs but high at short SOAs, thus, describing the process leading to a Type B masking function, and will be named "Factor B" subsequently. Figure 4.4 also shows the factor scores for each participant. Some participants have high scores on Factor A and low scores on Factor B, others vice versa. Still others have high and low scores on both factors, respectively.

\section{Correlation of Mask Bias With Individual Factor Scores}

To investigate a possible relation of Mask Bias $C_{M}$ with individual masking performance an ANCOVA was conducted with SOA as repeated-measures independent variable and the individual factor scores as covariates. 
Table 4.1: Experiment 2.1. Factor Analysis -- factor loadings for each SOA.

\begin{tabular}{rccc}
\hline SOA & Factor 1 & Factor 2 & communalities \\
\hline $24 \mathrm{~ms}$ & -0.036 & 0.905 & 0.820 \\
$36 \mathrm{~ms}$ & 0.816 & 0.566 & 0.986 \\
$48 \mathrm{~ms}$ & 0.950 & 0.172 & 0.932 \\
$60 \mathrm{~ms}$ & 0.936 & -0.008 & 0.876 \\
$72 \mathrm{~ms}$ & 0.922 & -0.200 & 0.890 \\
\hline sum of squares & 3.295 & 1.208 & \\
proportion variance & 0.659 & 0.242 & \\
cumulative variance & 0.659 & 0.901 & \\
\hline
\end{tabular}

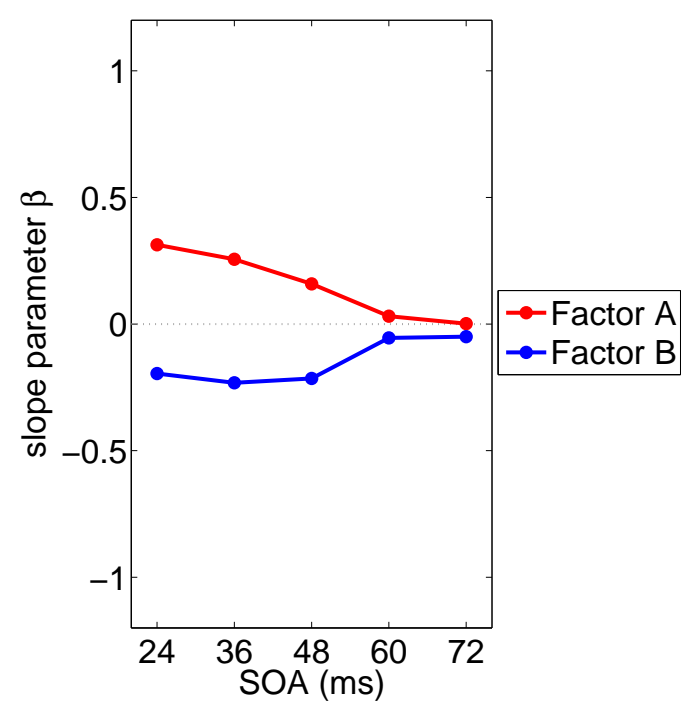

Figure 4.5: Experiment 2.1. Slope parameter $\beta$ over SOAs with Mask Bias $C_{M}$ values correlated with Factor A scores and Factor B scores.

There is no significant main effect of $\operatorname{SOA}(F(4,68)=0.52, p=.722)$, but significant interactions of SOA with Factor A scores $(F(4,68)=44.29, p<.001)$ and of SOA with Factor B scores $(F(4,68)=55.19, p<.001)$. There are main effects for Factor A scores $(F(1,17)=555.25$, $p<.001)$ and Factor B scores $(F(1,17)=76.21, p<.001)$. The slope of the regression line of the correlation between factor scores and $C_{M}$ values for each SOA is termed the slope parameters $\beta$ ), plotted in Figure 4.5 (page 42). The deeper the regression line, the higher is $\beta$, and the more strong correlate the values. The slope between $C_{M}$ values and Factor A scores are positive for short SOAs, meaning that a high factor score on Factor A leads to a high positive Mask Bias in short SOAs, and decreasing with increasing SOA (approaching zero), meaning that the a high factor score on Factor A does not predict the Mask Bias in long SOAs. The slope with Factor B scores is inverted-it is negative with short SOAs, meaning that a high factor score 
(a)

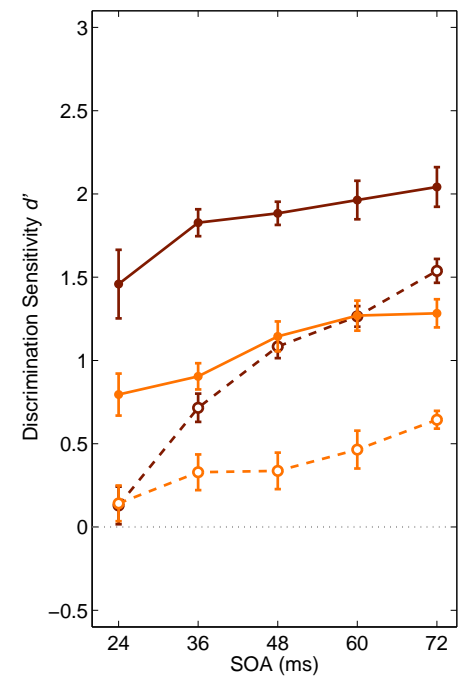

(b)

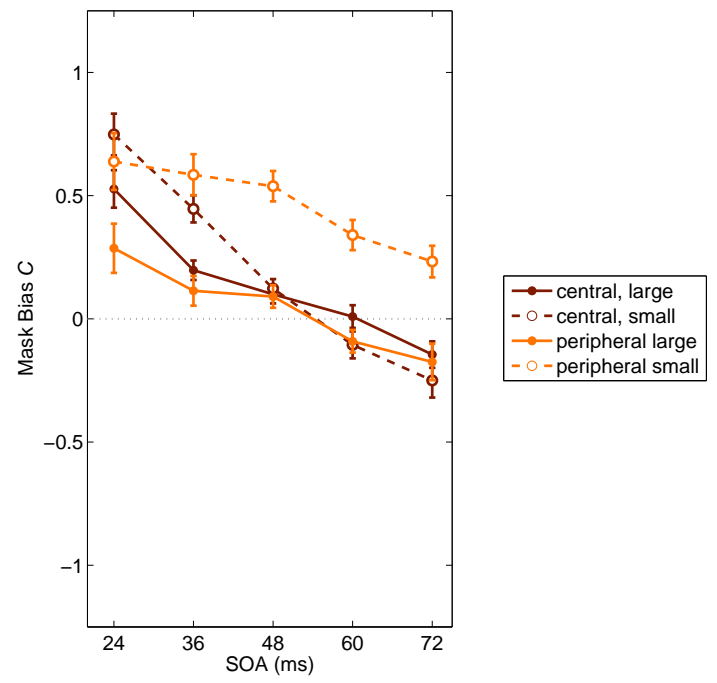

Figure 4.6: Experiment 2.2. Grand averages of (a) Discrimination Sensitivity $d^{\prime}$ and (b) Mask Bias $C_{M}$. Error bars represent the within-subject standard error (Loftus \& Masson, 1994).

on Factor B leads to a low Mask Bias for short SOAs, and is increasing with increasing SOA (approaching zero), meaning that a high factor score on Factor B does not predict the Mask Bias in long SOAs.

\subsubsection{Experiment 2.2}

\section{Discrimination Sensitivity}

Figure 4.6a (page 43) shows the Discrimination Sensitivity $d^{\prime}$ of Experiment 2.2. For all conditions Sensitivity is increasing with increasing SOA, thus, showing a Type A masking function (main effect SOA: $F(4,76)=20.08, p<.001$ ). However the level and the slope differ among conditions. In general, Sensitivity is higher for centrally than for peripherally presented stimuli (main effect Position $F(1,19)=51.89, p<.001$ ) and is higher for large than for small stimuli (main effect Size $F(1,19)=79,83, p<.001$ ). More specifically, Sensitivity for centrally presented large stimuli is higher than for centrally presented small stimuli and this difference is larger in short SOAs than in long SOAs, and for peripheral presented large stimuli Sensitivity is larger than for peripherally presented small stimuli (two-way interaction Size x SOA: $F(4,76)=3.45, p=.031$; two-way interaction Position x SOA: $F(4,76)=4.93, p=.010)$. In addition, masking function depends on presentation position as well as on stimulus size (three- 


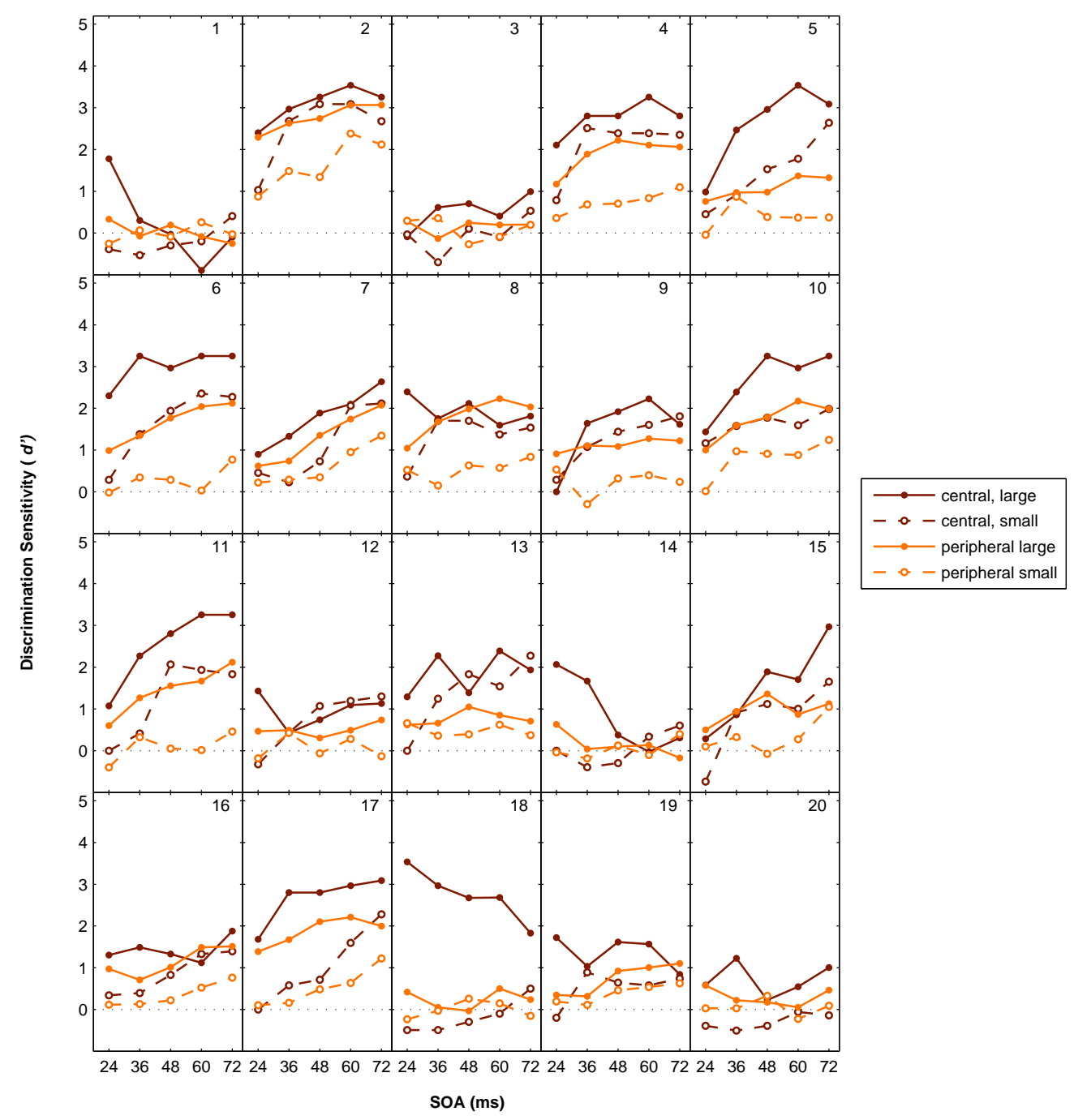

Figure 4.7: Experiment 2.2. Discrimination Sensitivity $d^{\prime}$, individual plots [1-20].

way interaction Position $x$ Size x SOA: $F(4,76)=6.25, p<.001)$. Individual masking functions with all conditions are shown in Figure 4.7 (page 44).

\section{Correlation of Discrimination Sensitivity With Individual Factor Scores}

The analysis of covariance showed that the impact of Factor A scores and Factor B scores on the masking function is different over SOAs and for presentation position (three-way interaction Factor A scores x SOA x Position: $F(4,68)=6.15, p<.001$; three-way interaction Factor B scores $\mathrm{x}$ SOA x Position: $F(4,68)=5.96, p<.001)$ as well as for stimulus size (three-way interaction Factor A scores $\mathrm{x}$ SOA x Size: $F(4,68)=4.86, p=.001$; three-way interaction Factor B scores $x$ SOA x Size: $F(4,68)=11.50, p<.001)$. 


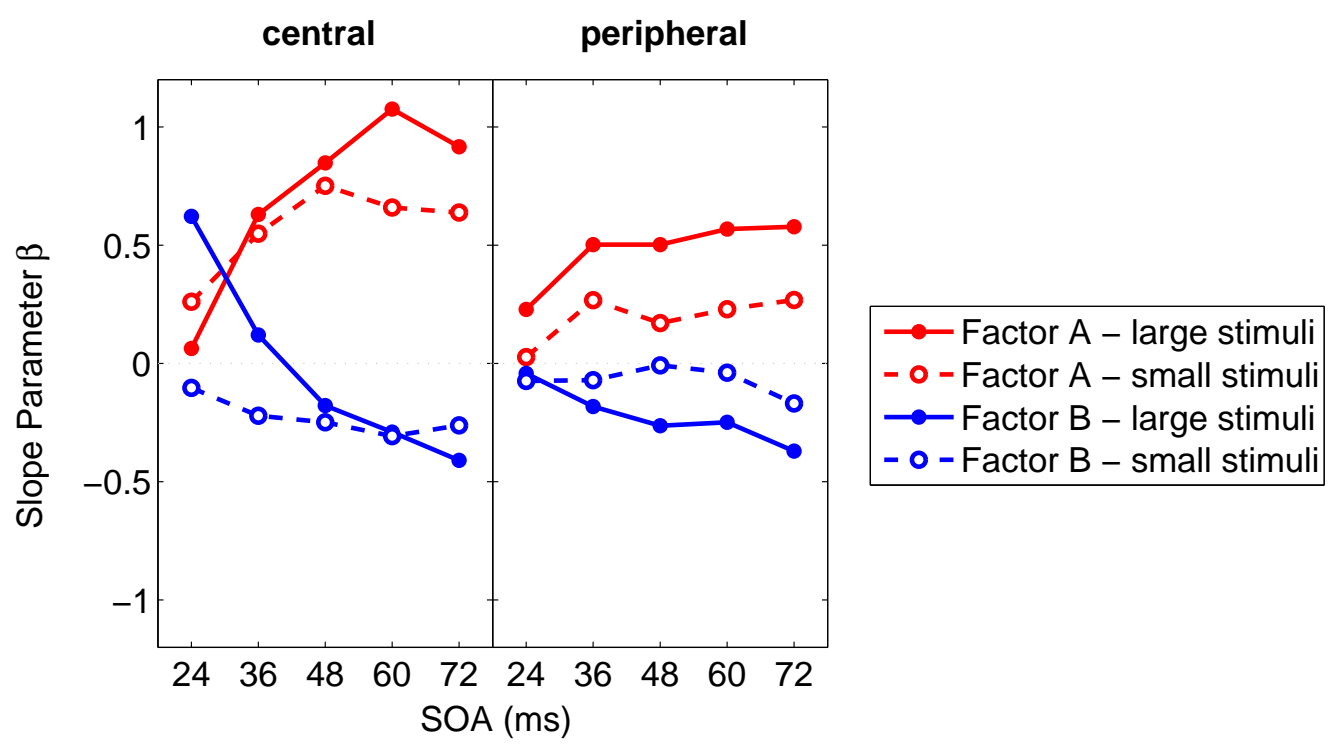

Figure 4.8: Experiment 2.2. Slope parameter $\beta$ over SOAs, with Discrimination Sensitivity $d^{\prime}$ values correlated with Factor A scores and Factor B scores for centrally (left-hand side) and peripherally presented stimuli (right-hand side).

The correlation of the factor scores with $d^{\prime}$ values are shown in Figure 4.8 (page 45). For large stimuli presented centrally, the correlation of of Factor A scores with $d^{\prime}$ values is increasing with increasing SOA, starting at zero and increasing with a peak correlation the SOA of $60 \mathrm{~ms}$. The correlation with the corresponding Factor B starts positive at 0.6 for the shortest SOA and is decreasing with increasing SOA, becoming negative until -0.4. A high factor score on Factor A predicts a high Sensitivity for intermediate and long SOAs, but not for the shortest SOA. In contrast, a high factor score on Factor B predicts a high Sensitivity in the shortest SOA, is less predictive for intermediate SOAs and predicts a low Sensitivity for long SOAs. Here, the shortest SOA is most remarkable as Factor B is higher than Factor A, emphasizing the special role of this factor in this SOA. In the peripherally presented large stimuli no such crossing can be observed. Factor A correlations are increasing with increasing SOA, while Factor B correlations are decreasing. The impact of both factors is weaker than in the central condition. The same pattern occurs for the centrally presented small stimuli. The peak correlation for Factor A is at the SOA of $48 \mathrm{~ms}$. For peripherally presented small stimuli the correlation with Factor B is for all SOAs between -0.3 and 0 , meaning that under these conditions the factor has no impact on Sensitivity. The impact of Factor A is increasing with increasing SOA with a peak correlation at the $36 \mathrm{~ms}$ SOA. However the overall impact is less strong as for the other conditions. 


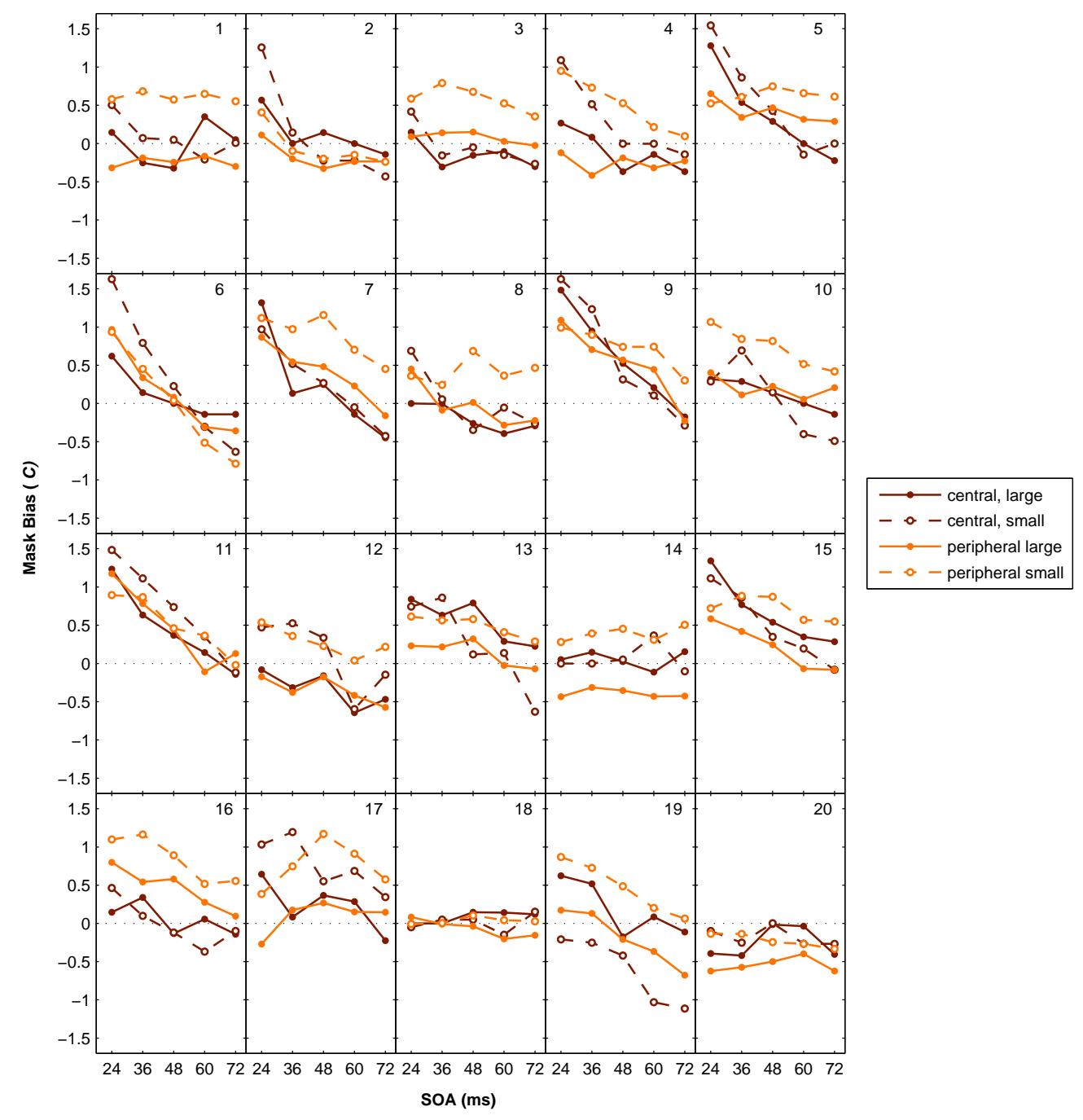

Figure 4.9: Experiment 2.2. Mask Bias $C_{M}$, individual plots [1-20].

\section{Mask Bias}

The Mask Bias is shown in Figure 4.6b (page 43), it is positive in short SOAs and decreasing with increasing SOA for all conditions (main effect SOA: $F(4,76)=, p<.001$ ). It is higher in peripherally presented small stimuli than in peripherally presented large stimuli over all SOAs, and it is higher in centrally presented small stimuli than in centrally presented large stimuli, especially in short SOAs (two-way interaction Size x SOA: $F(4,76)=4.87, p=.002$ ). The Mask Bias is more positive for peripherally presented small stimuli than for centrally presented small stimuli and is slightly more positive for peripherally presented large stimuli than for centrally presented large stimuli (two-way interaction Position x SOA: $F(4,76)=11.74, p<.001$ ). In addition, Mask Bias depends on presentation position as well as on stimulus size (three-way 


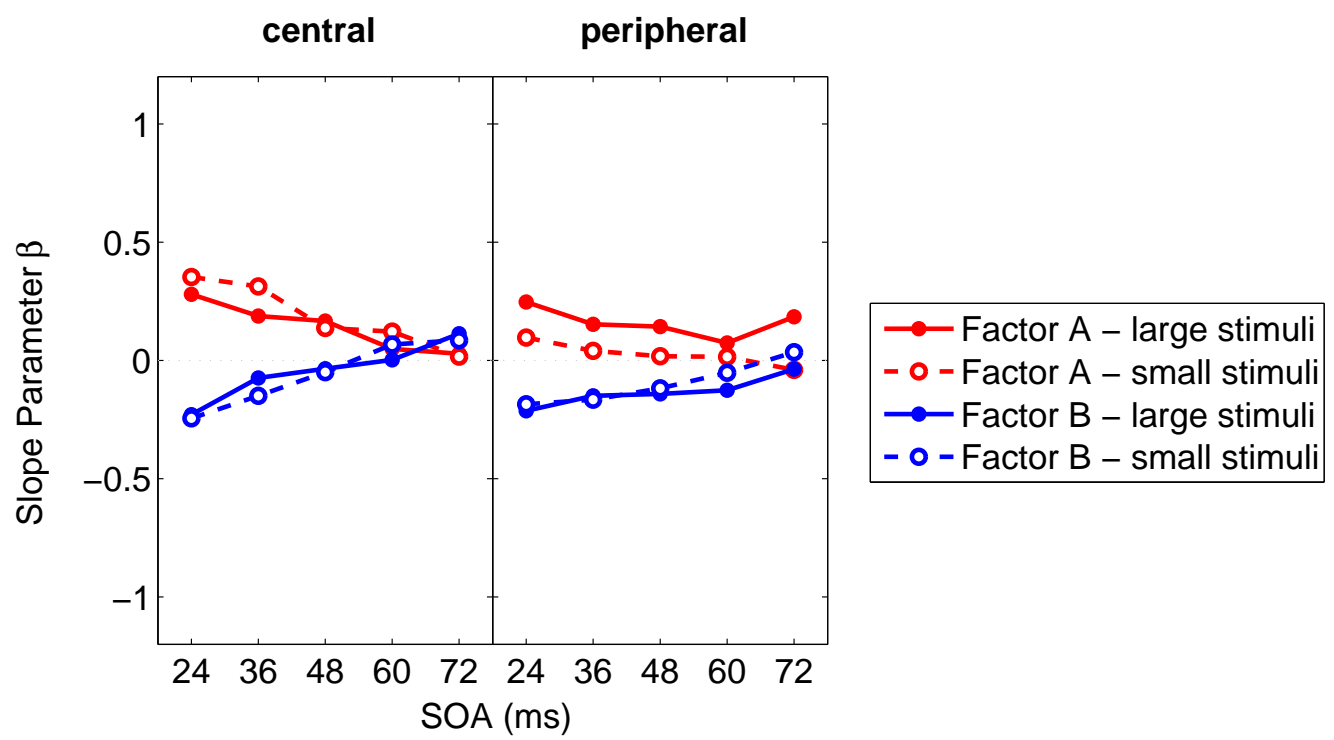

Figure 4.10: Experiment 2.2. Slope parameter $\beta$ over SOAs, with Mask Bias $C_{M}$ values correlated with Factor A scores and Factor B scores for centrally (left-hand side) and peripherally presented stimuli (right-hand side).

interaction Position x Size x SOA: $F(4,76)=7.76, p<.001)$. Individual functions with all conditions are shown in Figure 4.9 (page 46) .

\section{Correlation of Mask Bias With Individual Factor Scores}

The correlations of the factor scores with $C_{M}$ values are shown in Figure 4.10 (page 47). The correlation of Factor A and B scores with the Mask Bias $C_{M}$ do hardly differ between conditions. In short SOAs it is positive for Factor A and decreasing with increasing SOA, indicating that a high factor score on Factor A predicts a high Mask Bias in short SOAs (two-way interaction Factor A scores x SOA: $F(4,68)=4.50, p=.023)$. For Factor B it is negative in short SOAs and is increasing with increasing SOA, indicating that a high factor score on Factor B predicts a low Mask Bias in short SOAs (two-way interaction Factor B scores x SOA: $F(4,68)=6.81$, $p=.005$ ). For centrally presented stimuli the impact of both factors for small stimuli is slightly greater than for large stimuli but differs not over SOAs. For peripherally presented small stimuli the correlation with Factor A is near zero over all SOAs, so here the factor score does not predict the Mask Bias. But the course over SOAs does not differ between presentation positions (three-way interaction Factor A scores x SOA x Position: $F(4,68)=3.05, p=.050$; three-way interaction Factor B scores $x$ SOA x Position: $F(4,68)=1.69, p=.193$ ) or stimulus sizes (threeway interaction Factor A scores x SOA x Size: $F(4,68)=1.77, p=.179$; three-way interaction Factor B scores $x$ SOA x Size: $F(4,68)=0.98, p=.396)$. 
(a)

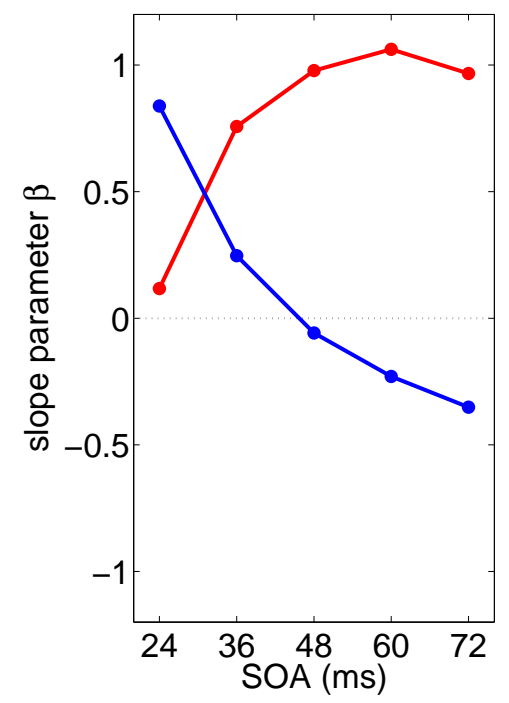

(b)

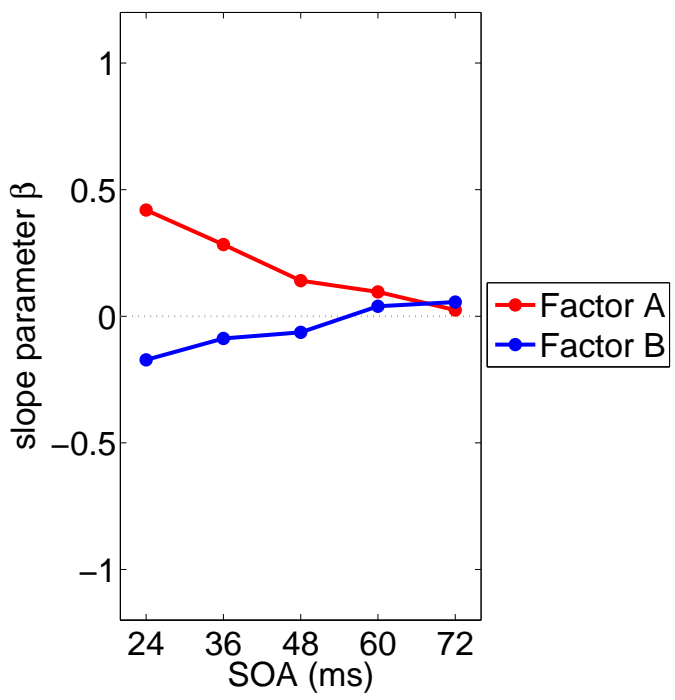

Figure 4.11: Experiment 2.3. Slope parameter $\beta$ over SOAs, with (a) Discrimination Sensitivity $d^{\prime}$ and (b) Mask Bias $C_{M}$ values correlated with Factor A scores and Factor B scores.

\subsubsection{Experiment 2.3}

\section{Discrimination Sensitivity}

Comparing the masking functions of Experiment 2.3 (Figure $4.2 \circ$, page 39) with Experiment $2.1(\bullet)$, shows that all participants kept their type of masking function. In some participants not only the course but also the level of performance (e.g. number 1, 11, or 14). In other participants the type of course is mostly the same except for an increase of the level of performance. This performance increase can be either equally distributed over all SOAs (e.g. number 2, or 4) or especially occurs in either long (e.g. number 7, 8, or 9) or short SOAs (e.g. number 16). The Discrimination Sensitivity of the grand average is equally distributed over all SOAs like in Experiment 2.1 but the performance level increased. The ANOVA confirms these observations: There is no main effect for $\operatorname{SOA}(F(4,76)=0.10, p=.981)$, but for Experiment $(F(1,19)=22.93, p<.001)$, and no significant interaction (two-way interaction SOA x Experiment: $F(4,76)=2.18, p=.079)$.

The ANCOVA reveals a main effect for Factor A scores $(F(1,17)=29.20, p<.001)$, but not for Factor B scores $(F(1,17)=0.37, p=.554)$ and SOA $(F(4,68)=1.73, p=.154)$. Both correlations for Factor A and Factor B scores with $d^{\prime}$ values become significant (2-way interaction Factor A scores x SOA: $F(4,68)=24.69, p<.001$; 2-way interaction Factor B scores x SOA: $F(4,68)=38.63, p<.001$ ). The slope parameter $\beta$ is shown in Figure $4.11 \mathrm{a}$ (page 48). For Factor $\mathrm{A}$ it is increasing with SOA, meaning that the a high factor score on Factor A predicts a high 
Mask Bias in intermediate and long SOAs. For Factor B it is positive in short SOAs, decreases with increasing SOA and becomes negative in long SOAs, meaning that a high factor score on Factor B does predict a high Sensitivity in short SOAs and low Sensitivity in long SOAs.

\section{Mask Bias}

The grand average of the Mask Bias (Figure $4.3 \circ$, page 40) is nearly identical to Experiment 2.1 $(\bullet$, two-way interaction Experiment x SOA: $F(4,76)=0.98, p=.0425)$. Individual masking functions differ in some participants but not in all. The ANCOVA revealed a main effect, for Factor A scores $(F(1,17)=9.66, p=.006)$, and SOA $(F(4,68)=23.56, p<.001)$, but not for Factor B scores $(F(1,17)=0.53, p=.479)$. The correlation of Factor A and Factor B scores with $C_{M}$ values differs across SOAs (2-way interaction Factor A x SOA: $F(4,68)=9.95, p<.001$; 2-way interaction Factor B scores x SOA: $F(4,68)=3.50, p=.038$ ). The slope parameter $\beta$ is shown in Figure 4.11b (page 48). For Factor A it is positive in short SOAs and decreasing with SOA approaching zero, meaning that a high factor score on Factor A predicts a high Mask Bias in short SOAs. For Factor B it is slightly negative for short SOAs and is increasing with SOA approaching zero, meaning that a high factor score on Factor B predicts partially a low Masking Bias in short SOAs. For long SOAs the loading on factor scores does not predict Masking Bias.

\subsection{Discussion}

\subsubsection{Experiment 2.1}

This experiment aimed to obtain inter-individual differences in the Discrimination Sensitivity using them for the factor analysis, whose factors represent potential processes involved in metacontrast masking. Like in Study 1 inter-individual differences in metacontrast masking occurred, which were further used for a factor analysis, which revealed two underlying latent variables explaining $90 \%$ of total variability. The Factor A variable is increasing with increasing SOA, explaining mainly data of intermediate and long SOAs, and the Factor B variable is decreasing with SOA, explaining mainly data of short SOAs. As described in Study 1 these factors may be regarded as reflecting two independent (perceptual) processes that jointly determine the shape of the masking function. The impact of each process depends on SOA and the specific weight of each participant.

The positive correlation between $C_{M}$ values and Factor A scores and the negative correlation between $C_{M}$ values and Factor B scores in short and intermediate SOAs show that a strong process A results in a positive Mask Bias and strong process B results in a negative Mas Bias 
in these SOAs. The null correlation in long SOAs shows that both processes have no special impact on the manifestation of the Mask Bias.

\subsubsection{Experiment 2.2}

This experiment examined the processes involved in metacontrast masking behave when stimulus presentation position and size are manipulated. The interaction of size and eccentricity described by Bridgeman and Leff (1979) could be replicated partially. In foveally presented stimuli masking was stronger in small stimuli than in large stimuli, this is in line with their study. But in the periphery small stimuli are stronger masked than large stimuli as well, this is in contradiction to Bridgeman and Leff (1979). The finding that the SOA with the strongest masking increases with stimulus size could not be replicated as well. In this study the strongest masking occurs at the shortest SOA for both stimulus sizes. A pure interaction of stimulus size and eccentricity could not be found, however, a three-way interaction with SOA, implicating the relevance of the SOA in this interaction. So, the masking function depends on stimulus size as well as eccentricity.

Based on the data of the correlation, both underlying processes are less effective when stimuli were presented in the periphery, nevertheless process A predominates. The process B is most effective in large stimuli presented centrally, while the process A works for small stimuli as well. Assuming that the segregation process is represented by process $\mathrm{A}$ it can be postulated that in the periphery stimuli were processed more segregated with increasing effectiveness the longer the SOA is, which seems natural as the temporal resolution is higher in the peripheral visual field and segregation easier with longer gaps between both presentations. Furthermore, for small stimuli the segregation process seems to prevail over the integration process. Assuming the integration process is represented by process B it can be further postulated that target and mask are better integrated when stimuli are large and presented centrally, but only for short SOAs. For long SOAs the segregation process prevails.

It is remarkable that in all conditions (both sizes and both eccentricities) the averaged masking functions have a Type A course although it could be expected that large and centrally presented stimuli more likely favor Type B masking functions. Two explanations are possible: The first possibility is that, as in Experiment 2.1 more participants showed a Type A than a Type B masking function, the grand average of Experiment 2.2 is more likely Type A shaped. However, having a look on individual data in Experiment 2.2, participants who showed a Type B masking function in Experiment 2.1 have trouble to maintain this type in all conditions of Experiment 2.2 showing masking functions without much variability over SOAs either at low or intermediate level, or even increasing with increasing SOA. Additionally, the factor analysis revealed a clear Type B process. This supports the second possibility that the segregation process is facilitated 
as most of the conditions favor this processing. Especially peripherally presented stimuli are more likely processed via the segregation process as receptive fields are larger in the peripheral retina, resulting in a stronger convergence of the light, enabling a higher Sensitivity to light and a higher ability to detect movements in the periphery. Therefore, the criterion content of movement detection is most beneficial in peripherally presented stimuli. This may explain the strong impact of the Mask Bias in this study, in contrast to Study 1, where it was not affected. So, the manifestation of this criterion content and the tracing of the transient channel, due to the experimental conditions, may result in an overall Type A processing.

As the spatial resolution decreases with increasing eccentricity, stimuli have to be enlarged to obtain the same performance. This adjustment of stimulus size can be calculated with the cortical magnification factor (see e.g. Lingnau \& Vorberg, 2005; Slotnick, Klein, Carney, \& Sutter, 2001). In this study the magnification was not considered as at an eccentricity of $5^{\circ}$ visual angle stimuli would have been too large (taking a magnification factor of 13.4 stimuli the largest mask stimulus would have been $64.3^{\circ}, 74.8 \mathrm{~cm}$ at a viewing distance of $67 \mathrm{~cm}$ ). Thus, stimuli with the same objective size are represented with a subjectively different size in the cortex, when they were presented either in the fovea or in the periphery, which influences the Discrimination Sensitivity. However, without magnification, results of this study confirm the decrease of Sensitivity with increasing eccentricity.

\subsubsection{Experiment 2.3}

There is a general performance increase over all SOAs, indicating a training effect. With a closer look on the individual data all participants maintained their masking function shown in Experiment 2.1 and most of the participants show an increase in their performance level. This is remarkable as in Study 1 all participants shifted their course to a Type B masking function. This further supports the assumption of context effects in Study 1, which seem not to be present in Study 2.

Mask Bias does not differ between Experiment 2.1 and Experiment 2.3, indicating that the impact of both factors on the Mask Bias has not changed due to Experiment 2.2.

\subsection{Conclusion}

In this study inter-individual differences in metacontrast masking and their interaction with varied stimulus sizes and presentation eccentricities were investigated. Stimulus size and eccentricity have interacting effects on the masking function. These results partly replicate those of Bridgeman and Leff (1979). Discrimination performance increases with stimulus size and decreases with distance from the Fovea. In addition, the size effect and the position effect de- 
pend on the individual factor scores for both, Factor A and Factor B. The integration process represented by Factor B is most effective when large stimuli are presented foveally, while it seems to be ineffective in small stimuli and in the visual periphery. The segregation process represented by Factor A is most effective in long SOAs, independent of stimulus size. As stimuli presented peripherally are difficult to discriminate, the ability to perceptually segregate both stimuli is advantageous. 


\section{Study 3 - Effect of Spatial Attention}

The focus of attention usually is where the focus of the eye is. By moving the eye the retinal focus shifts, and along with it attention is shifted to the new locus as well. However, it is also possible to shift ones attention to an area different from the eye's fixation. This is called covert attention. Here, the attention is either concentrated to a smaller area or distributed over a larger one. When this attentional shift is triggered by an onset of a stimulus, for example in the periphery, the perception of this stimulus is processed fast and transient (Montagna, Pestilli, \& Carrasco, 2009). Attention can also be voluntarily shifted within the visual field and may be sustained for some seconds. These shifts of spatial attention enable priory processing of the stimuli in the attended locations in trade-off for a lower processing of the unattended locations. In this way the visual system can overcome its limited capacity in processing due to high metabolic costs of cortical computations. Montagna et al. (2009) showed that performance decreased in unattended locations and concluded that limited processing resources affect early vision, even when there is no location uncertainty. Attention is often manipulated by using visual cues indicating a location where stimuli will appear (valid cues). However, these cues can also be invalid indicating to the wrong location or neutral being inconclusive. Using such spatial cues Bruchmann, Hintze, and Mota (2011) showed that valid cues have no benefit for performance, but invalid cues have costs, which means that performance decreases. In masking experiments the masking effect decreases when stimuli are presented in the area where attention is shifted to (e.g. Bashinski \& Bacharach, 1980), as stimuli are processed more early. In contrast, masking effect increases when attention is distributed over a larger area as the strength of this attention effect is weakened. Beck and Ambler (1973) compared the performance to discriminate differences in line slope and line arrangement with distributed or concentrated attention using eight letters arranged circularly (and therefore are presented peripherally). Letters were Ts and one disparate letter could be included, which was either a tilted $\mathrm{T}$ (same line arrangement, different orientation) or an L (different line arrangement, same orientation). The attention was manipulated by dot indicators, either a single dot where attention had to be concentrated, or two dots either adjacent or on the opposite site of the circle. Between both, attention had to be distributed. They found that with distributed attention accuracy is higher for the orientation task than for the arrangement task, while with concentrated attention there was no performance difference. 


\section{Study 3 - Effect of Spatial Attention}

In her study from 1966 Weisstein manipulated the area over which participants had to share their attention and examined the target identification performance over varied SOAs (see also Francis, 2003). In the second experiment of the study two conditions are of interest: one letter was presented in either 4 or 8 possible positions. Letters were either D or $\mathrm{O}$ (height $0.62^{\circ}$ visual angle), presented for $20 \mathrm{~ms}$, followed by a ring mask for $50 \mathrm{~ms}$, surrounding the letter. The SOA was varied between 0 and $120 \mathrm{~ms}$. The four locations were arranged in a horizontal line, two on each side of the fixation cross with an eccentricity of $0.61^{\circ}$ for the inner letters and $1.73^{\circ}$ for the outer letters. Unfortunately, it is not further described how the eight letters were arranged within the visual field. Participants had to correctly identify the letter. They knew if four or eight letters would be presented, and therefore could spread their attention as much as necessary to encompass all letters. For the stimulus presentation a tachistoscope was used. When all stimuli were presented simultaneously (SOA $0 \mathrm{~ms}$ ), and when the SOA was at least $30 \mathrm{~ms}$ long, performance did not differ between both conditions. But when the SOA was 10 or $20 \mathrm{~ms}$ the performance for eight possible positions was lower than for four possible positions. The effect of masking in the former was strongest at $10 \mathrm{~ms}$, and in the latter at $30 \mathrm{~ms}$. Therefore, the minimum of the masking function shifted to shorter SOAs the broader the attention was distinguished. In addition, the effects of attention seemed to be only in the decreasing branch of the U-shaped masking function.

In the study of Enns and Di Lollo (1997) the authors examined in their first experiment the differences in metacontrast masking when stimuli were presented in one or three possible locations. They used a small $\left(0.62^{\circ}\right.$ visual angle) black diamond shape as target and a larger diamond shaped metacontrast mask. Both stimuli were presented for $30 \mathrm{~ms}$ and the SOA was varied between 0 and $300 \mathrm{~ms}$. The target missed either the right or the left point, participants had to discriminate the side of the missing point. Stimuli were presented either centrally as the only possible location, or in three possible locations, namely central or with $3^{\circ}$ eccentricity either on the left or the right side of the center (only trials with target and mask presented at the same location will be regarded). So, there was a spatial uncertainty where stimuli will appear. Two comparisons are of importance: The masking function of centrally presented stimuli in one versus in three possible locations, and the masking function of centrally versus peripherally presented stimuli in three possible locations. As there is no masking effect for simultaneously presented stimuli, only positive SOAs will be considered. For the condition one central location the masking function is increasing with increasing SOA (Type A) with a minimum at $45 \mathrm{~ms}$ and nearly no masking effect for SOAs longer than $90 \mathrm{~ms}$. When the presentation location was uncertain, the masking function was U-shaped (Type B) with a minimum at $90 \mathrm{~ms}$ (and a remarkable masking effect for the SOA of $135 \mathrm{~ms}$ ). Conclusively, with more location uncertainty the minimum of the masking function shifted towards longer SOAs, changing from Type A to 
Type B. When stimuli were presented in the periphery, masking is very strong for the SOAs 45, 90, and $135 \mathrm{~ms}$ and weaker for the SOA of $300 \mathrm{~ms}$. Between the first three SOAs the performance differences are minor, but a minimum at $90 \mathrm{~ms}$ could be discerned. Conclusively, when the presentation location is uncertain, masking is stronger in the periphery than in the fovea, and differences are most prominent in the shortest SOA (ergo in the decreasing branch).

This study aims to determine how the processes involved in metacontrast masking behave when sustained endogenous attention is either focused on one presentation area or has to be distributed over a larger region. The study is composed of three experiments: In the first Experiment 3.1 the SOA is varied in a metacontrast paradigm. It is expected to obtain individually different masking functions of each participant, which can be used for the factor analysis, whose factors describe the individual differences and represent the involved processes. In the second Experiment 3.2 the presentation position of the stimuli and the size of the area to be attended is varied in addition to the SOA. Here, stimuli are presented either in one of four possible peripheral positions or foveally. It was blockwise varied if stimuli appeared predictably in one of the five positions, enabling the participant to shift their attention in this smaller area while focusing the fixation point in the center, or if stimuli appeared unpredictably, randomized in one of the five positions, forcing the participant to distribute their attention over the whole presentation area. Stimulus size was kept constant. The individual factor scores of the factor analysis of Experiment 3.1 were correlated with the Discrimination Sensitivity and the Mask Bias of Experiment 3.2 to obtain a relation measure, which provides information about the contribution of the specific processes on the individual masking functions. In the third Experiment 3.3 individual masking functions of the first Experiment are compared to those during the Experiment 3.2 with the same conditions to investigate possible training or context effects.

\subsection{Methods}

\subsubsection{Participants}

Twenty healthy participants (10 male) participated in this experiment. All but six were righthanded, and their mean age was $\mathrm{M}=23.2$ ( $\mathrm{SD}=2.7$ ), ranging from 19 to 28 .

\subsubsection{Experimental Set-Up}

The study is composed of three experiments with four sessions in total. (However the third experiment is a mere comparison between performances of the first and second experiment.) In all sessions they had to fulfill the target identification task. The first Experiment 3.1 includes the first session with 720 trials in 12 blocks (144 repetitions per SOA). They were presented as 


\section{Study 3 - Effect of Spatial Attention}

shown in Figure $2.1 \mathrm{~b}$ (page 10) with the exception that the SOA was only varied between $24 \mathrm{~ms}$ and $72 \mathrm{~ms}$.

The second Experiment 3.2 includes sessions two to four with 2400 trials in total. The stimulus size was as described in General Methods (Section 2.2, page 9). The SOA was varied between $24 \mathrm{~ms}$ and $72 \mathrm{~ms}$. The eccentricity of the presentation positions were varied and could be either central $\left(0^{\circ}\right.$ eccentricity) or above, below, left to, or right to the center with $5^{\circ}$ eccentricity (see Figure 4.1, page 37). In half of the blocks the stimulus position was randomized, in the other half of blocks stimulus position was announced at the beginning of the block and thus predictable. Per SOA and predictability 240 trials were presented.

To control that participants fixate the fixation cross in the center of the display and in this way observe stimuli in the periphery out of focus (for the conditions in the eccentricity), the Eyelink 1000 eyetracker (SR-Research, binocular mode, $1000 \mathrm{~Hz}$ temporal resolution) was used to monitor eye positions and movements. The eye tracking was calibrated at the beginning of the experiment and after breaks on demand. Each trial starts only after participants fixate the fixation cross. Viewing distance was $67 \mathrm{~cm}$. To keep the visual angle constant the absolute size of the stimuli in Experiment 3.1 were reduced (in relation to the absolute size with $100 \mathrm{~cm}$ viewing distance).

\subsubsection{Data Analysis}

For all experiments the Discrimination Sensitivity $d^{\prime}$ and the Mask Bias $C_{M}$ were calculated using the signal detection theory by Macmillan and Creelman (2005) as described in Chapter 2.4. With the data of Experiment 3.1 a factor analysis was conducted as described in Chapter 2.4. For this, data of Experiment 3.2 were averaged over sessions for each conditions. Statistical analyses were run with presentation positions and attentional focus as independent variables. In a first step, analysis of variances (ANOVA) were conducted to examine the overall effects of presentation positions and attentions. In a second step, to test for the effect of individual differences, a full factorial repeated measures analysis of covariance (ANCOVA) was conducted with the within-subject factors SOA, presentation positions and attentions, and the two continuous between-subject covariates Type A Score and Type B Score obtained in Experiment 3.1. ANCOVA models included all pure within-subject effects, the main effect for each covariate, and the interactions of each covariate with all within-subject effects. Thus, all effects including an interaction of both covariates were excluded. The slope of the regression of $d^{\prime}$ and $C_{M}$ values with the factor scores, the slope parameter $\beta$, is calculated for each SOA, representing the correlation between $d^{\prime}$ and $C_{M}$ values with the factor scores. For Experiment 3.3, the comparison of masking functions before and after training, Sensitivity and Mask Bias data of the condition 


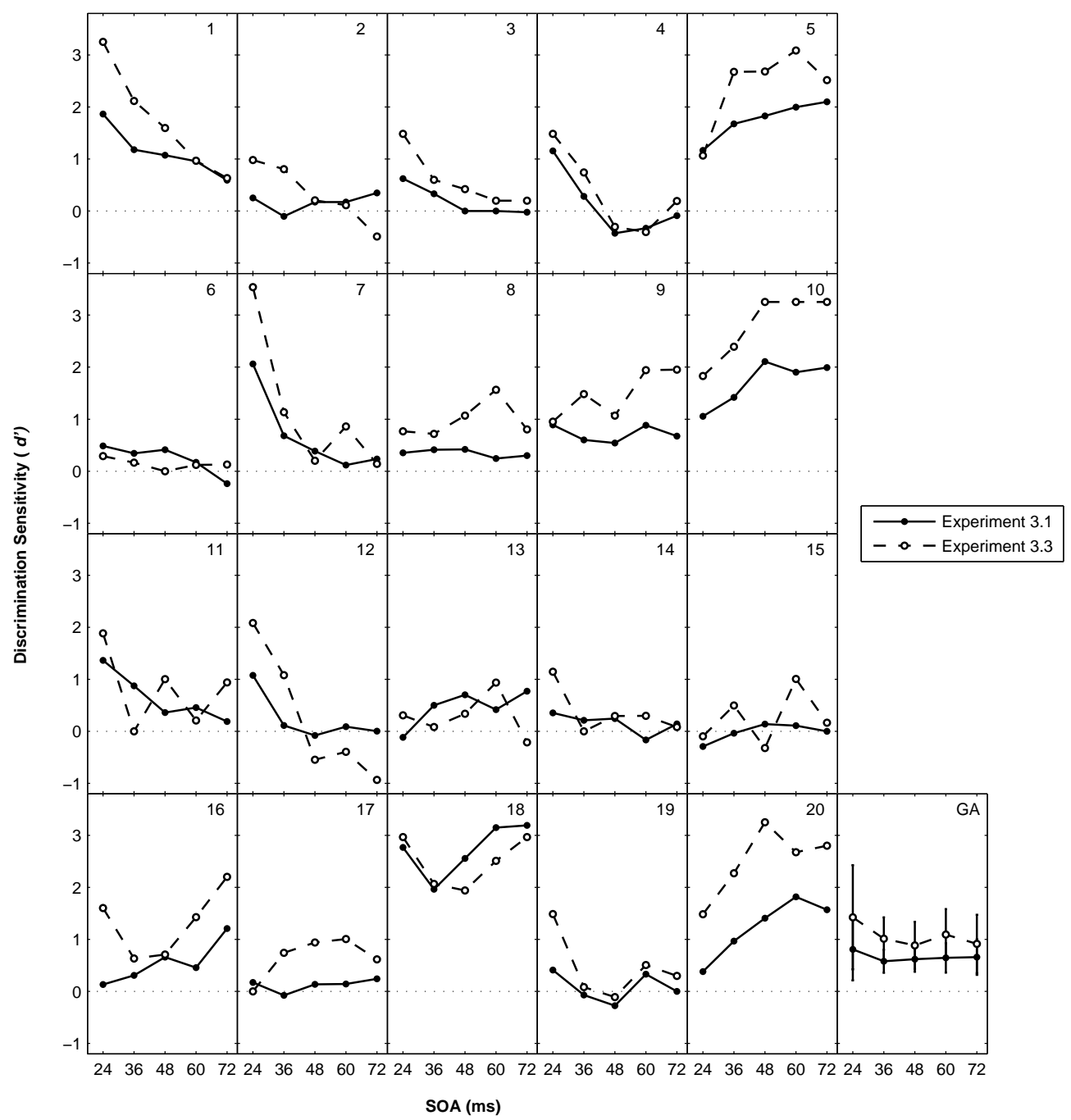

Figure 5.1: Experiment $3.1(\bullet)$ and $3.3(\circ)$. Discrimination Sensitivity $d^{\prime}$ (individual plots [1-20] and grand average [GA]). Error bars represent the within-subject standard error (Loftus \& Masson, 1994).

central presented, predictable of Experiment 3.2 was used. Statistical analysis was conducted using R (R Core Team (version 3.0.1) [Computer software], 2013).

\subsection{Results}

\subsubsection{Experiment 3.1}

\section{Discrimination Sensitivity and Mask Bias}

The individual masking functions and Mask Biases with the grand averages are shown in Figure $5.1(\bullet$, page 57) and $5.2(\bullet$, page 58). Comparable to Study 1 and 2 there is substantial inter- 


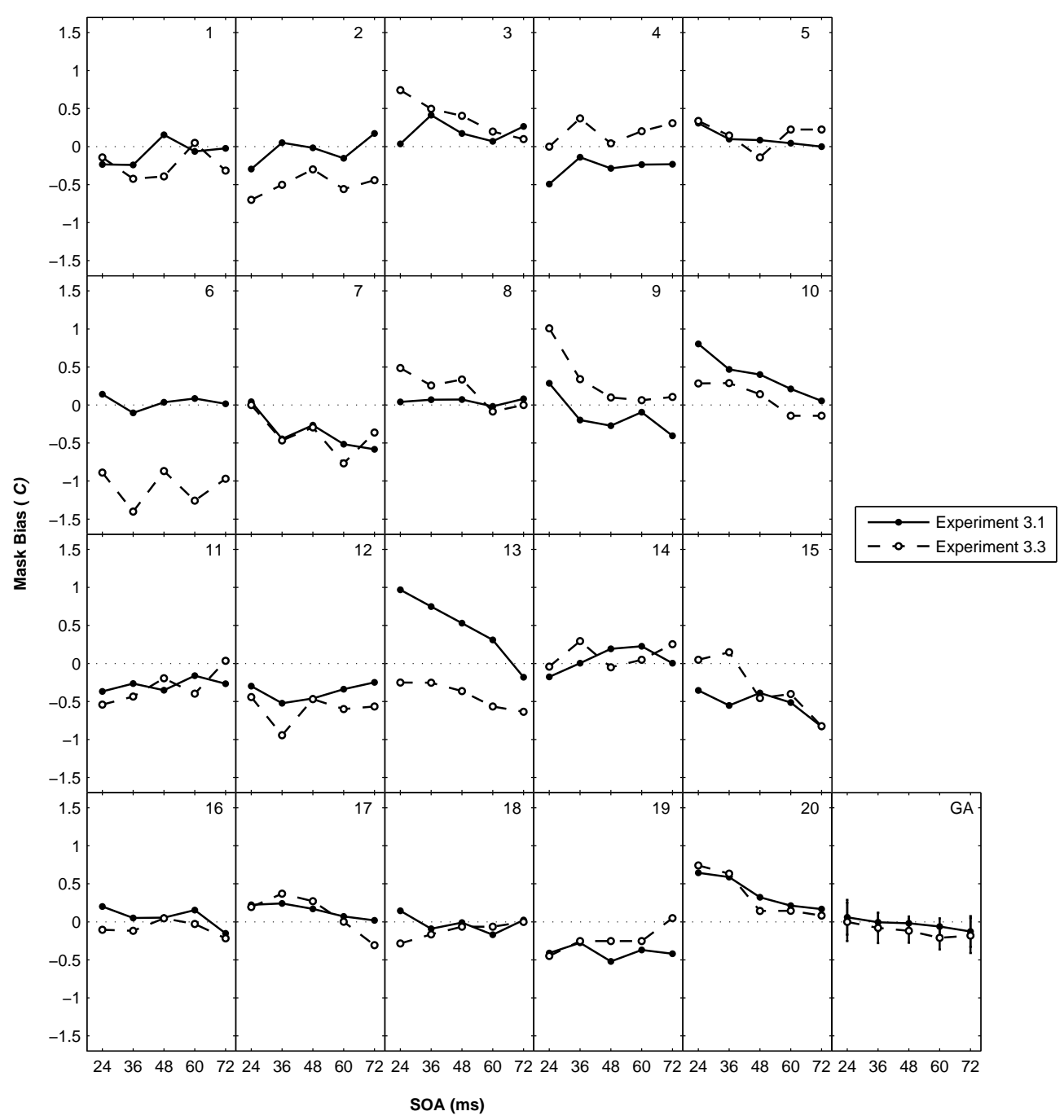

Figure 5.2: Experiment $3.1(\bullet)$ and $3.3(\circ)$. Mask Bias $C_{M}$ (individual plots [1-20] and grand average [GA]). Error bars represent the within-subject standard error (Loftus \& Masson, 1994).

individual variability with regard to the slopes and the levels. The averages over all datasets are inconclusive without variability over SOAs. Accordingly, two one-way repeated-measures ANOVAs showed no significant effect of SOA neither on Sensitivity $d^{\prime}(F(4,76)=0.92, p=.384)$ nor on Mask Bias $C_{M}(F(4,76)=3.00, p=.062)$.

\section{Factor Analysis}

The maximum likelihood factor analysis revealed two factors with eigenvalues $>1$, which together explain $90 \%$ of total variance $\left(\chi^{2}(1)=3.25, p=.071\right.$, Table 5.1, page 60$)$. In contrast, the one-factor solution only explains $79 \%$ of total variability, and thus fits the data only poorly $\left(\chi^{2}(5)=16.52, p=.006\right)$. As only five SOAs were examined a three-factor-analysis was not 


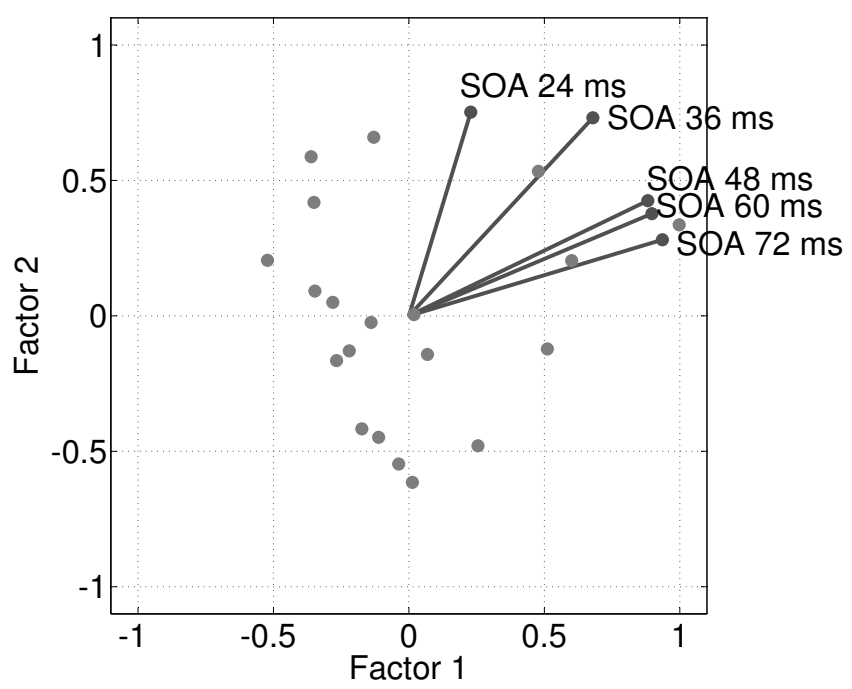

Figure 5.3: Experiment 3.1. Factor analysis - biplot with factor loadings (-) for each SOA and factor scores for each participant $(\bullet)$. (Please note that scales refer to factor loadings.)

possible. Therefore, two factors are most appropriate for the data. Loadings of Factor 1 are increasing over SOAs, while loadings of Factor 2 are decreasing (Table 5.1, page 60, and Figure 5.3, page 59). As the results are in line with Study 1 and 2 and the assumptions are the same, Factor 1 and 2 are named "Factor A" and "Factor B", respectively. Figure 5.3 also shows the factor scores for each participant. Here again, individuals have high or low scores on both factors.

\section{Correlation of Mask Bias With Individual Factor Scores}

To investigate a possible relation of Mask Bias $C_{M}$ with individual masking performance an ANCOVA was conducted with the SOA as repeated-measures independent variable and the individual factor scores as covariates. For visualization the slope parameter $\beta$ is shown in Figure 5.4 (page 60). Factor $A$ is slightly positive in short SOAs and decreasing with increasing SOA. However, this interaction turned out to be not significant (two-way interaction Factor A scores x SOA: $F(4,78)=2.57, p=.090$ ), therefore, higher Factor A scores do not lead reliably to greater Mask Bias values in short SOAs, and are not predictive about the Mask Bias in long SOAs. The impact of Factor B is near zero over all SOAs implicating that the Factor B scores are irrelevant for manifestation of the Mask Bias (two-way interaction Factor B scores x SOA: $F(4,78)=0.36, p=.708)$. 
Table 5.1: Experiment 3.1. Factor analysis -- factor loadings for each SOA.

\begin{tabular}{rccc}
\hline SOA & Factor 1 & Factor 2 & communalities \\
\hline $24 \mathrm{~ms}$ & 0.228 & 0.752 & 0.618 \\
$36 \mathrm{~ms}$ & 0.679 & 0.731 & 0.995 \\
$48 \mathrm{~ms}$ & 0.882 & 0.425 & 0.958 \\
$60 \mathrm{~ms}$ & 0.897 & 0.377 & 0.948 \\
$72 \mathrm{~ms}$ & 0.936 & 0.281 & 0.955 \\
\hline sum of squares & 2.971 & 1.502 & \\
proportion variance & 0.594 & 0.300 & \\
cumulative variance & 0.594 & 0.895 & \\
\hline
\end{tabular}

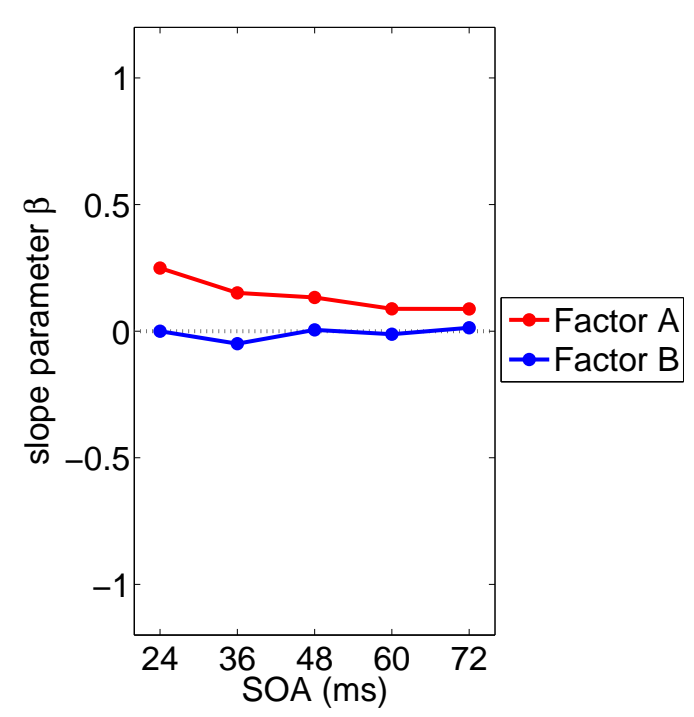

Figure 5.4: Experiment 3.1. Slope parameter $\beta$ over SOAs, with Mask Bias $C_{M}$ values correlated with Factor A scores and Factor B scores.

\subsubsection{Experiment 3.2}

\section{Discrimination Sensitivity}

Discrimination Sensitivity $d^{\prime}$ is shown in Figure 5.5a (page 61). Sensitivity is higher in centrally presented stimuli than in peripherally presented stimuli, independent of SOA (main effect Position: $F(1,19)=19.95, p<.001)$. But Sensitivity does not differ between predictable stimuli, that is, when the presentation position is known and attention could be focused on one position, and unpredictable stimuli, that is, when the presentation position is unknown and attention has to be distributed over all five positions, independent of SOA (main effect Predictability: $F(1,19)=1.55, p=.229$ ). Centrally presented stimuli show a Type B masking functions, here, for unpredictable positions only slightly with a minimum at SOA $60 \mathrm{~ms}$, and predictable 
(a)

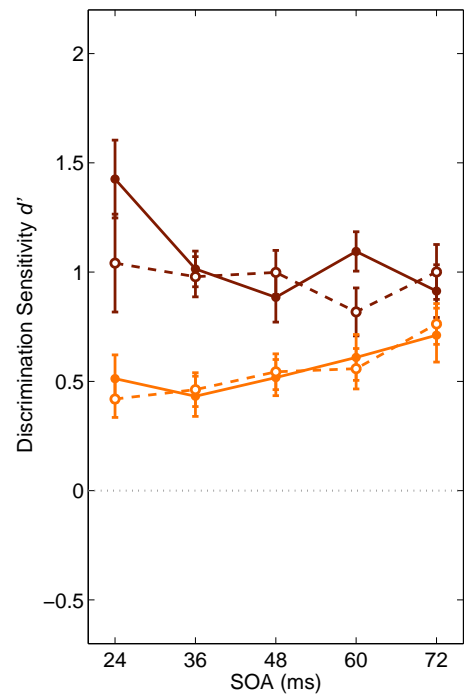

(b)

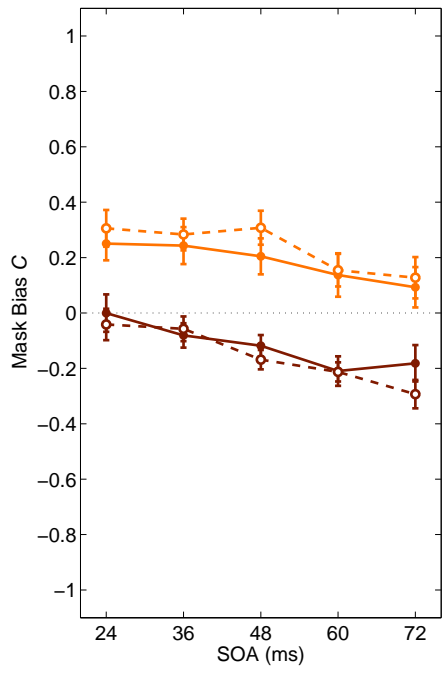

$\rightarrow$ central, predictable - $\circ$ - central, non-predictable peripheral, predictable - peripheral, non-predictable

Figure 5.5: Experiment 3.2. Grand average of (a) Discrimination Sensitivity $d^{\prime}$ and (b) Mask Bias $C_{M}$. Error bars represent the within-subject standard error (Loftus \& Masson, 1994).

positions with a minimum at SOA $48 \mathrm{~ms}$. In contrast, peripherally presented stimuli show a Type A masking function, with a minimum at SOA $24 \mathrm{~ms}$ for unpredictable positions and a minimum at SOA $36 \mathrm{~ms}$ for predictable positions. This difference between positions (two-way interaction Position x SOA: $F(4,76)=3.84, p=.033$ ) and predictabilities (two-way interaction Predictability x SOA: $F(4,76)=4.25, p=.004)$, are higher in short SOAs than in long SOAs. But Sensitivity is not dependent on both, position and predictability. The effect of predictability is not different between positions (two-way interaction Position x Predictability: $F(1,17)=2.60$, $p=.125$ ), and there is no further interaction with SOA (three-way interaction Position $\mathrm{x}$ Predictability x SOA: $F(4,76)=0.98, p=.425)$. Individual masking functions with all conditions are shown in Figure 5.6 (page 62).

\section{Correlation of Discrimination Sensitivity With Individual Factor Scores}

The slope parameter $\beta$ of the correlations of the factor scores with $d^{\prime}$ values are shown in Figure 5.7 (page 63). The impact of Factor A is increasing with increasing SOA, for both positions and both predictabilities. While the slope is greater in the central condition than in the peripheral condition (three-way interaction Factor A scores x Position x SOA: $F(4,68)=4.37, p=.021$ ), there is no difference between predictable and unpredictable stimulus positions (two-way interaction Factor A scores x Predictability: $F(1,17)=1.86, p=.191)$ and no further interaction with SOA (three-way interaction Factor A scores x Predictability x SOA: $F(4,68)=0.53, p=.712$ ). 


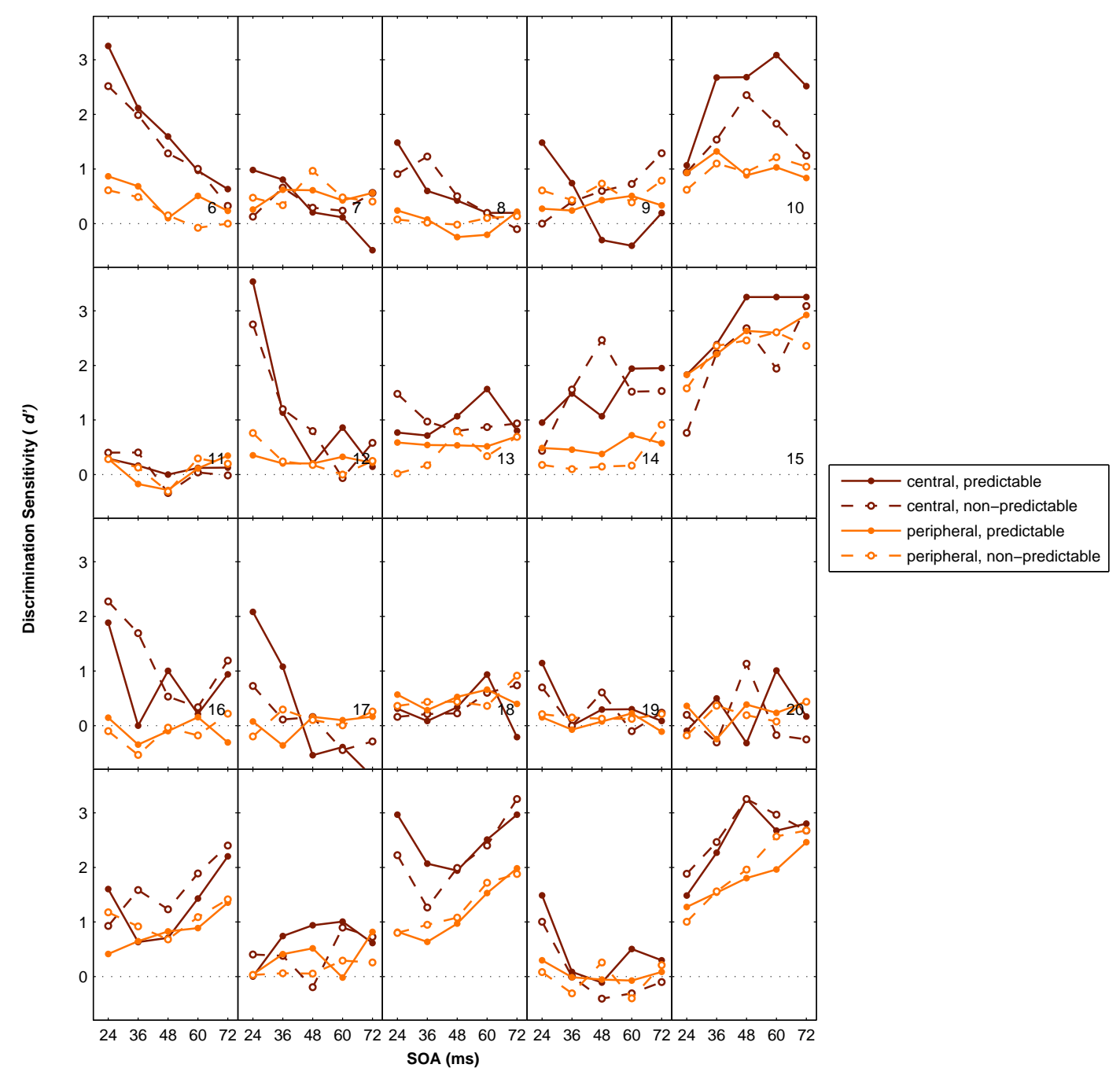

Figure 5.6: Experiment 3.2. Discrimination Sensitivity $d^{\prime}$, individual plots [1-20].

In centrally presented stimuli the impact of Factor B is decreasing with increasing SOAs, but in peripherally presented stimuli the Factor B has no impact at all. The ANCOVA reveals a significant difference between positions (two-way interaction Factor B scores x Position: $F(1,17)=14.83, p=.001$ ), but there is no further interaction with SOA (three-way interaction Factor B scores x Position x SOA: $F(4,68)=1.79, p=.183)$. Furthermore, the impact of Factor $B$ does not differ between predicted and unpredicted stimulus positions (two-way interaction Factor B scores x Predictability: $F(1,17=0.66, p=.429)$ and there is no interaction between predictability and SOA (three-way interaction Factor B scores $\mathrm{x}$ Predictability $\mathrm{x}$ SOA: $F(4,68)=0.19, p=.943)$. The impact of both factors does not depend on all three factors (four-way interaction Factor A scores x Position x Predictability x SOA: $F(4,68)=0.55$, $p=.699$; four-way interaction Factor B scores $\mathrm{x}$ Position $\mathrm{x}$ Predictability $\mathrm{x}$ SOA: $F(4,68)=0.45$, $p=.774)$. 


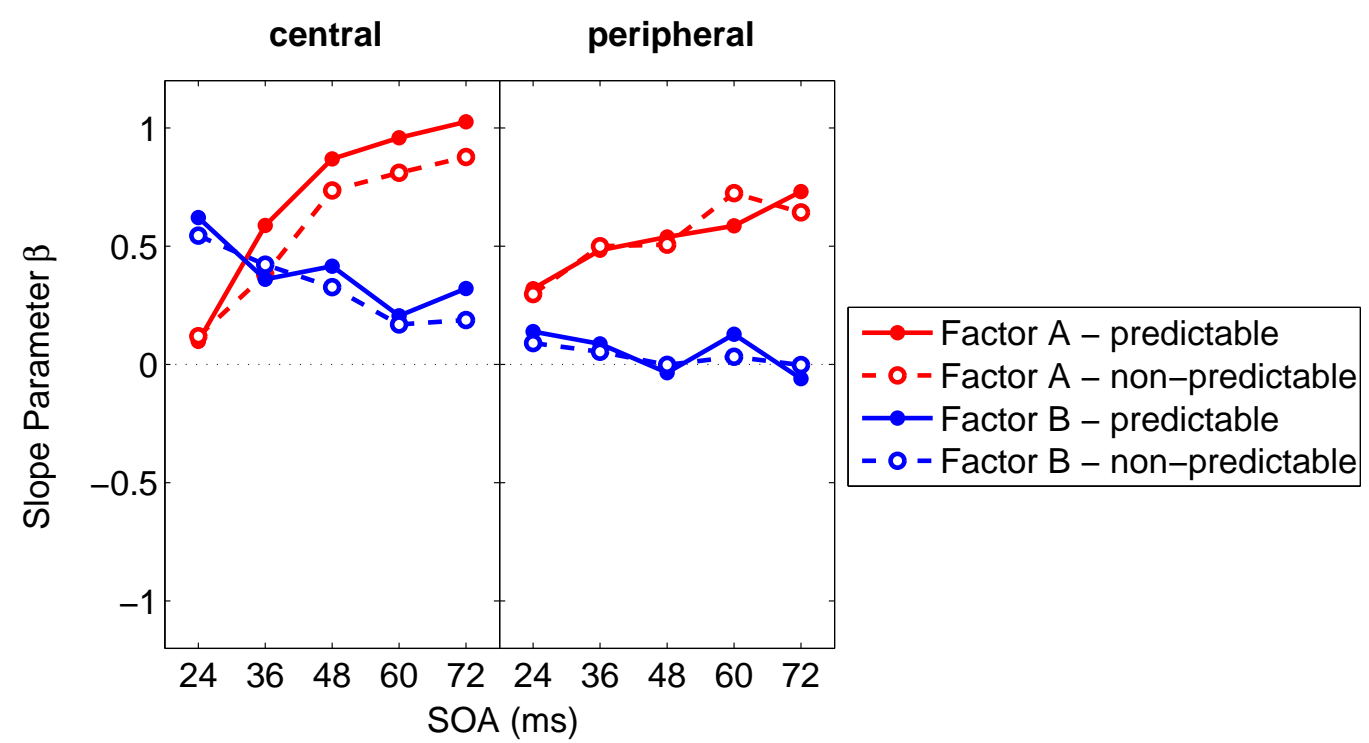

Figure 5.7: Experiment 3.2. Slope parameter $\beta$ over SOAs, with Discrimination Sensitivity $d^{\prime}$ values correlated with Factor A scores and Factor B scores for centrally (left-hand side) and peripherally presented stimuli (right-hand side).

\section{Mask Bias}

The Mask Bias for all conditions is shown in Figure 5.5b (page 61). Mask Bias is positive for peripherally presented stimuli and negative for centrally presented stimuli (main effect Position: $F(1,19)=15.34, p<.001)$ but there is no difference between predicted and unpredicted stimulus positions (main effect Predictability: $F(1,19)=0.04, p=.845$ ). Although slightly decreasing with increasing SOA the Mask Bias has no significant variability over SOAs (two-way interaction Position x SOA: $F(4,76)=0.60, p=.667$; two-way interaction Predictability x SOA: $F(4,76)=0.63, p=.644)$. Furthermore, the Mask Bias does not depend on both predictability and position (three-way interaction Position $\mathrm{x}$ Predictability $\mathrm{x}$ SOA: $F(4,76)=0.90, p=.470$ ). Individual functions with all conditions are shown in Figure 5.8 (page 64) .

\section{Correlation of Mask Bias With Individual Factor Scores}

The slope parameter $\beta$ of the correlations of the factor scores with $C_{M}$ values are shown in Figure 5.9 (page 65). Both factors have only little impact on the manifestation of the Mask Bias and there are no variations between positions, predictabilities, or over SOA (two-way interaction Factor A scores x Position: $F(1,17)=0.07, p=.790$; two-way interaction Factor A scores x Predictability: $F(1,17)=0.02, p=.890$; two-way interaction Factor A scores $\mathrm{x}$ SOA: $F(4,68)=0.48, p=.749$; two-way interaction Factor B scores $\mathrm{x}$ Position: $F(1,17)=1.89$, $p=.187$ : two-way interaction Factor B scores $x$ Predictability: $F(1,17)=0.02, p=.878$; two- 


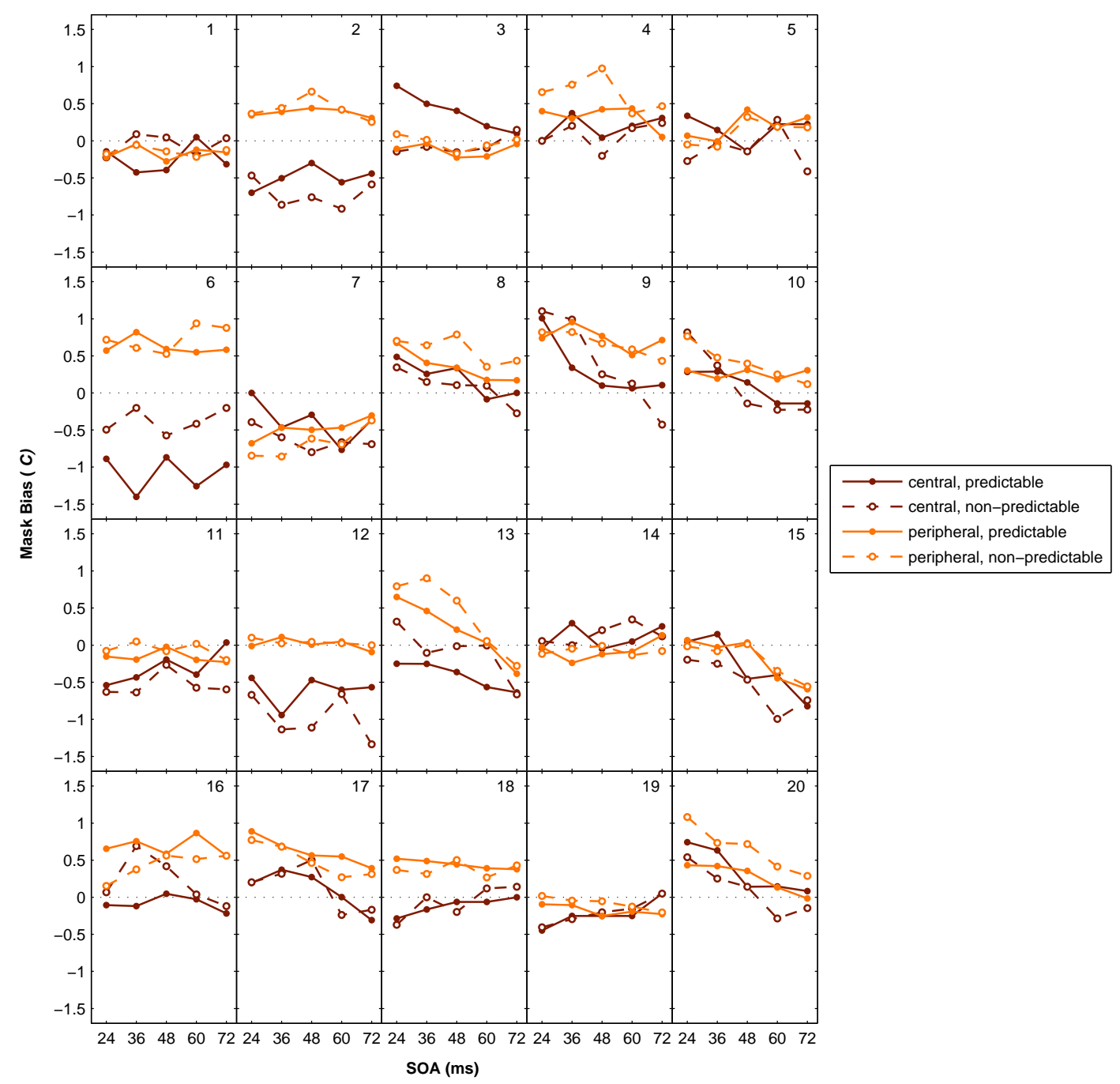

Figure 5.8: Experiment 3.2. Mask Bias $C_{M}$, individual plots [1-20].

way interaction Factor B scores x SOA: $F(4,68)=2.17, p=.081)$. So, the value of individual factor scores does not predict the Mask Bias in any condition.

\subsubsection{Experiment 3.3}

In comparison to Experiment 3.1 individual masking functions (Figure 5.1, page 57) mainly do not differ, but most of the participants have an performance increase in some or all SOAs. Regarding the grand average there is a general performance increase (main effect Experiment: $F(1,19)=20.70, p<.001$ ), but variability over SOAs does not increase (two-way interaction Experiment x SOA: $F(4,76)=1.79, p=.140)$.

The averaged Mask Bias $C_{M}$ (Figure 5.2, page 58) does not differ from Experiment 3.1 either, not in its course over SOA (two-way interaction Experiment $\mathrm{x}$ SOA: $F(4,76)=0.63, p=.639$ ), 


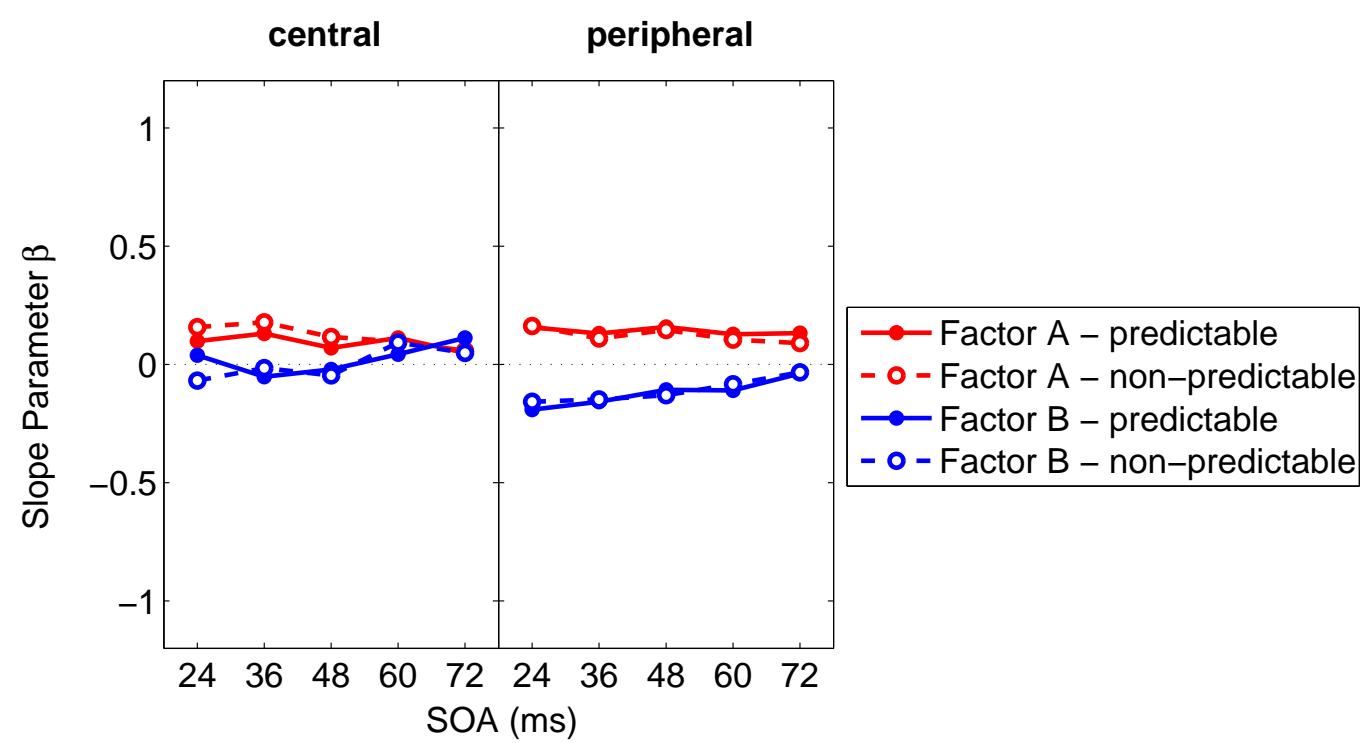

Figure 5.9: Experiment 3.2. Slope parameter $\beta$ over SOAs, with Mask Bias $C_{M}$ values correlated with Factor A scores and Factor B scores for centrally (left-hand side) and peripherally presented stimuli (right-hand side).

and not in its level (main effect Experiment: $F(1,19)=1.02, p=.326$ ). Individual Biases did not change in course over SOAs but in some participants the Bias decreased or increased.

\subsection{Discussion}

\subsubsection{Experiment 3.1}

This experiment aimed to obtain inter-individual differences in the Discrimination Sensitivity using them for the factor analysis, whose factors represent potential processes involved in metacontrast masking. Like in Study 1 and 2 inter-individual differences in metacontrast masking occurred, which were further used for a factor analysis, which revealed two underlying latent variables explaining $90 \%$ of total variability. The Factor A variable is increasing with increasing SOA, explaining mainly data of intermediate and long SOAs, and the Factor B variable is decreasing with SOA, explaining mainly data of short SOAs. As described in Study 1 these factors may be regarded as reflecting two independent (perceptual) latent processes that jointly determine the shape of the masking function. More specifically, it is assumed that Factor A represents a segregation process, resulting in a percept of two succeeding stimuli, and that Factor B represents an integration process, resulting in a percept of a simultaneous, conjoint presentation of both stimuli. Both factors have no impact on the manifestation of the Mask Bias $C_{M}$ in Experiment 3.1 . 


\section{Study 3 - Effect of Spatial Attention}

\subsubsection{Experiment 3.2}

In this experiment the sustained endogenous attention was either focused on one presentation area or had to be distributed over a larger region to determine how the processes are involved in metacontrast masking in such conditions. Sensitivity differs between eccentricities. It is higher for centrally presented stimuli than for peripherally presented stimuli, especially in short SOAs, indicating a higher masking effect in the periphery. This conforms results of Experiment 2.2. The masking function of the predictable, peripherally presented, intermediate-sized stimuli lies between those of large and small, peripherally presented stimuli of Experiment 2.3, indicating a reduction of Sensitivity similarly over SOAs the smaller stimuli become. The masking functions of centrally presented stimuli are less comparable to Experiment 2.2. In fact, it shows a quite different course. While in Experiment 2.2 both stimulus sizes have a Type A course, ranging from near 0 to approximately 1.5 for small stimuli and from approximately 1.5 to approximately 2 for large stimuli, the masking function of Experiment 3.2 has a Type B course, ranging from approximately 1.5 to approximately 1 (all values of $d^{\prime}$ ). However, comparing this condition with results of Experiment 2.3, as both used the same stimulus size, the Sensitivity is similar in all SOAs but the shortest (24 ms). Here, the Sensitivity is higher in Experiment 3.2 than in Experiment 2.3. This indicates that the masking functions are much more dependent on stimulus size in central than in peripheral presentation positions.

At first appearance the predictability of the presentation positions seem to have no benefit for Sensitivity. Masking functions are identical for predictable and unpredictable stimulus presentation locations. Thus, distributing the spatial attention over a large area in contrast to focusing attention on a smaller area does not affect the masking effect. An exception may be the SOA of $24 \mathrm{~ms}$ in the central condition. Here, the Sensitivity is higher in predictable than in unpredictable positions, indicating a special benefit of predictability in very short SOAs. Comparing these results with those of other studies is difficult as conditions are very different. Weisstein (1966) presented her stimuli in both conditions in the periphery, and with much less eccentricity. Therefore considering only the peripheral conditions, there was no significant difference between predictable and unpredictable positions. But beside statistics the minimum of the masking function where attention had to be more distributed over a larger area is at shorter SOAs than when attention had to be more focused on a smaller area, therefore replicating their findings (whilst taking into account that the effects presented here are much smaller). The study of Enns and Di Lollo (1997) has only two SOAs in common with Experiment 3.2. Their shortest SOA is $45 \mathrm{~ms}$ long and the following is $90 \mathrm{~ms}$ long, missing very short and intermediate long SOAs to compare results. Nevertheless, they found lower performance in peripheral than in central positions in trials with uncertainty as well. Furthermore, they observed that the minimum of the masking function for centrally presented stimuli is at longer SOAs when the presentation 
position is unpredictable than when it is predictable, which is in accord with the present experiment as well. Conclusively, when considering the minima of the masking functions it shifts toward longer SOAs when attention is more distributed and stimuli are presented in the fovea than when attention is more focused, and it shifts toward shorter SOAs when attention is more distributed and stimuli are presented in the periphery than when attention is more focused.

As the impact of Process A is high in long SOAs it can be assumed that this process is the more activated the longer the SOA is, and this is independent on eccentricity even though more pronounced in the fovea. By contrast, Process B is more activated in short SOAs than in long SOAs and only in foveally presented stimuli. It is irrelevant in the periphery, independent of SOA. Therefore, the masking functions in the central conditions may result due to a conjoint activation of both processes: the high Sensitivity in short SOAs due to a high activation of Process B, and the relatively high Sensitivity in long SOAs due to a high activation of Process A. In the peripheral condition the low Sensitivity in short SOAs may due to the lack of Process B and the higher Sensitivity in long SOAs due to the high activation of Process B. These characteristics are comparable with those to Study 2. However, both processes seem to be unrelated to attention manipulations. The impact of Process A is slightly lower when attention is distributed than when it is narrowed and stimuli are presented foveally (except for the shortest SOA where it is identical). When stimuli are presented peripherally Process A's impact does not differ between these two conditions. The impact of Process B does not differ between the two attention manipulations, neither when stimuli were presented foveally nor when they were presented peripherally. The segregation process depends predominantly on the SOA than on the eccentricity being effective in central as well as peripheral stimulations. In contrast, the integration process looses influence in the periphery being most effective in the foveal visual field. The strength of both processes was not affected by attention manipulations.

The Mask Bias is relatively low in all conditions. However, it is slightly positive in peripherally presented stimuli, especially in short SOAs, indicating that apparent motions are less often perceived in short SOAs and therefore the response is given, according to the shape of the mask. In centrally presented stimuli, the Mask Bias is slightly negative, especially in long SOAs, indicating that the response is made contrary to the shape of the mask. Assuming that the percept of apparent motion is the stronger the longer the SOA is. This would indicate that participants experience a misleading percept of motion, which may rather be a sudden change than a fluent rotation or expansion. Both processes are not involved in the manifestation of the Mask Bias. It could be that the processes are not involved in the manifestation of the Mask Bias, or it could be that Mask Biases are to small to reveal correlation with both processes. In contrast to Study 2, the Process B has no effect in very short SOAs. The impact of both processes does not differ be- 


\section{Study 3 - Effect of Spatial Attention}

tween the two attention manipulations, neither when stimuli were presented foveally nor when they were presented peripherally.

It was expected that Process B has less impact when attention is distributed as fewer resources are available per possible location for the processing of details of the stimuli. It may be that attention was not that equally distributed or broad enough shared as it was envisaged. Here, small dots or light circles indicating the possible locations may help the participant. Alternatively (or additionally), a session or some blocks could be preceded training the equal distribution of attention over a large area to ensure the attention manipulation. Another possibility is that the appearance of the target immediately attract the transient attention toward this location so that stimuli are processed with undivided attention. Unfortunately, it stands to reason that this automatic mechanism could not be removed by additional training. It is recommended to conduct a further experiment with such adjustments to finally conclude that attention does not effect metacontrast masking in these conditions.

\subsubsection{Experiment 3.3}

In this experiment individual masking functions were compared to those of Experiment 3.1 to investigate possible training or context effects. The individual masking function did not change its type in the condition central, predictable due to the variation of spatial attention and eccentricity of presented stimuli, but general performance level increased slightly evenly over all SOAs, implicating a training effect independent of SOA. The Mask Bias does not change under these conditions and no impact of both processes arised.

\subsection{Conclusion}

In this study inter-individual differences in metacontrast masking and their interaction with varied transient endogenous attention and presentation eccentricities were investigated. The predictability of the presentation positions have statistically no benefit for Sensitivity. But when considering the minima of the masking functions, it shifts toward longer SOAs when attention is more distributed and stimuli are presented in the fovea than when attention is more narrowed. And it shifts toward shorter SOAs when attention is more distributed and stimuli are presented in the periphery than when attention is more narrowed. The segregation process represented by Factor A is most effective in long SOAs, independent of eccentricity. The integration process represented by Factor B is most effective in short SOAs and in the foveal visual field, while it seems to be ineffective in the visual periphery. The strength of both processes was no affected essentially by attention manipulations. 


\section{Study 4 - Effect of Attention}

In contrast to Study 3, where visual spatial attention was modulated, this study addresses another type of attention modulation, namely the voluntary set of focus of visual attention. Here, behavioral performance and neural processing are compared when areas or stimuli are specifically attended or not attended. It has to be dissociated from transient attention, which is an involuntary, automatic process where attention is shifted toward to due to a change of stimulus' features, a movement, its appearance or disappearance. It could be shown that such top-down attention modulation modulates the effect of metacontrast masking (Breitmeyer \& Öğmen, 2006; Ramachandran \& Cobb, 1995; Tata, 2002). Studies examining event-related potential (ERP) differences under attention manipulation are more rare. In the study of Stojanoski and Niemeier (2011) participants had to detect the target inside an array of gabor stimuli. The target was a loop of gabors associated by specific contours or by motion. The frequency of the contourdefined and motion-defined loop was altered blockwise so that participants adjust themselves to either detect contours or motions. When attending to specific features of the stimulus (contours or motions), which were at higher frequency, then perception of this feature is enhanced. Additionally, the authors found an ERP modulation around the time of the P1 component for motion stimuli in extrastriate areas but not for contour stimuli and later ERP modulations for contour but not for motion stimuli, which were associated with higher visual processes and which were assumed to occur in the lateral occipital complex (LOC).

The electrophysiological essentials of metacontrast masking were already studied long ago e.g. by Vaughan and Silverstein (1968), Andreassi, Simone, and Mellers (1976), or Bridgeman (1988). A more recent study by Railo and Koivisto (2009) compared ERPs in dependence of the visibility of the target by manipulating the strength of masking. Targets were either square or diamond shaped figures and the inner as well as outer contours of metacontrast mask were star-shaped. This mask results in a strong masking effect especially in the intermediate SOA of $50 \mathrm{~ms}$. This behavioral and physiological masking effect was compared to a pseudomask having star-shaped outer contours but a circular inner shape and therefore could not encompass contours of the target stimuli resulting in a lower masking effect as visibility is higher. With the pseudomask the ERPs were more negative (less positive) to the more visible targets than of the ERPs to the less visible targets of the metacontrast mask in the time range of 330-420 ms after 


\section{Study 4 - Effect of Attention}

target-onset over the posterior temporal lobes (T5, T6), followed by a late positive deflection (LP) between 450 and $700 \mathrm{~ms}$ after the target onset.

But there seem to be no physiological studies examining ERP differences with attention manipulations in metacontrast masking paradigm. However, there are several studies examining specific frequency bands in this issue, but this shall not be addressed here. From this perspective, the present study is a new approach in physiological research of metacontrast masking. Therefore, some methodological approaches of Tse, Martinez-Conde, Schlegel, and Macknik (2005) are used. Their fMRI study attempted to localize the neuronal correlates of visual awareness by using visual masking. They concluded that the circuits correlated to visual awareness of simple targets are restricted to the occipital lobe beyond V1 and V2. To control eye movements and foveal attention the participants had to respond to a slight change of the fixation point color, occurring randomly while masking stimuli were presented. In addition to the standard stimulation where target and mask stimuli were presented alternately (standard wave of invisibility), trials without the mask stimulus (target-only) and trials without the target stimulus (mask-only) were presented. These additional conditions were used for subtractions to gain knowledge about the amount of masking in each area. If the target response adds to the mask response in the standard condition and was not suppressed by the mask the difference would be less than zero. If the target response does not add to the mask response (difference equal or greater to zero) and therefore was suppressed correlating with the perception of the mask.

A pilot study showed specific electroencephalographic (EEG) activations for Type A and Type B participants indicating a modulation of visual processing by top-down influences. This study aims to replicated those findings and to examine the role of attention in the different processing of metacontrast masking stimuli. For this, two conditions were compared. In one condition the participant's attention was at the stimuli as usual in a metacontrast masking paradigm. In a second condition the same stimulation is used but participants have to ignore the stimuli (and focus their attention on the fixation point in the center instead). If both groups of participants already show functional differences in the latter condition (main effect of groups) it could be referred that participants have a different bottom-up processing. If an interaction between sessions and groups is found, there is evidence for a different top-down processing.

The study is composed of two experiments. The first Experiment 4.1 consists of two sessions as described above. The condition without attending stimuli (Experiment 4.1.1) is conducted before the condition with attention on stimuli (Experiment 4.1.2) to ensure that participants are naive to the individual phenomenological perceptions driven by the stimuli. In the first session (without attention) participants had to respond to a occasionally occurring color change of the fixation point (to keep their attention off the stimuli but within the same visual area), while in 
the second session (with attention) participants had to fulfill a target discrimination task. In both sessions the SOA is varied in a metacontrast paradigm and the EEG is recorded.

The second Experiment 4.2 consists of only one session, placed between the two sessions of Experiment 4.1. The SOA is varied in a metacontrast paradigm and stimuli were attended to fulfill a discrimination task. No EEG was recorded. This Experiment serves as training for Experiment 4.1.2 so that participants could manifest their type to gain distinct groups as best as possible and it was intended to be used for group classification in Experiment 4.1.

\subsection{Methods}

\subsubsection{Participants}

Eighty healthy, naive participants were recruited for this study. Several participants had to be excluded (criteria described in 6.1.4): one due to a neurological insult during Experiment 4.1.2, twelve as they did not attend all sessions, ten as their masking course could not be categorized to one of both groups, and four as the quality of their EEG recordings were not sufficient. Of the remaining 53 participants used for analysis were all but four right-handed, and their mean age was $\mathrm{M}=23.5$ ( $\mathrm{SD}=3.1$ ), ranging from 19 to 32 .

\subsubsection{Experimental Set-Up}

The study is composed of two experiments with three sessions in total. In the first and the third session (belonging to Experiment 4.1) an EEG was recorded. The trial course was as shown in Figure 2.1b (page 10) with two exceptions: First, in the first and third session only two SOAs were used, namely a short SOA with $24 \mathrm{~ms}$ and a long SOA with $72 \mathrm{~ms}$. In the second session (Experiment 4.2) five SOAs were used, varied between $24 \mathrm{~ms}$ an $72 \mathrm{~ms}$ in $12 \mathrm{~ms}$ steps. Second, a small, black fixation point $\left(0.15^{\circ}\right)$ instead of a fixation cross was used. The experimental set-up of session one and three was identical, only the task was altered. Per session 960 trials were presented in 24 blocks. In 96 trials both stimuli, target and mask, were presented and a color change from black (RGB: 0, 0, 0) to red (RGB: 139, 0, 0) appeared for $84 \mathrm{~ms}$. This color change could appear at one of three possible time points: (a) directly before the target, (b) directly after target (during the SOA and the beginning of the mask), or (c) during the mask with synchronized offsets of the mask and the color change. In 672 trials both stimuli, target and mask, were presented and no color change of the fixation point occurred. In 192 trials only the target was presented and a black fixation point instead of the mask. In 24 of these targetonly trials a color change appeared as described above and in the remaining 168 trials no color change occurred. All conditions were presented randomized over the session. 


\section{Study 4 - Effect of Attention}

The task in the first session was to focus the fixation point and detect an occasionally appearing color change to keep their attention off the metacontrast stimuli but on this visual area. Metacontrast stimuli had to be ignored. To respond that there was a color change the right control key had to be pressed, to respond that there was no color change the left control key had to be pressed. This is the condition without attention on the metacontrast stimuli. In the third session participants had to focus their attention on the metacontrast stimuli and perform the form identification task. They had to ignore the color change of the fixation point. To indicate that the target was a square the left control key had to be pressed, and to indicate that the target was a diamond the right control key had to be pressed. This is the condition with attention on the metacontrast stimuli. The condition without attention on stimuli (Experiment 4.1.1) is conducted before the condition with attention on stimuli (Experiment 4.1.2) to ensure that participants are naive to the individual phenomenological perceptions driven by the stimuli.

In the second session (Experiment 4.2) participants also performed the form identification task, where they had to identify the shape of the target by pressing the left control key on the keyboard to answer that it was a square, and by pressing the right control key to answer that it was a diamond. In total 720 trials were presented in 12 blocks with 144 repetitions per SOA. This Experiment served as training for Experiment 4.1.2 so that participants could manifest their type with more SOAs to gain distinct groups as best as possible and it was intended to be used for group classification for Experiment 4.1.

Participants gave their response $800 \mathrm{~ms}$ after mask offset to avoid neuronal motor response preparations. To indicate the the response time window the fixation point turned gray (RGB: $100,100,100)$. By giving the response the next trial starts. Participants were instructed in all sessions to fixate the fixation point and to avoid eye movements and blinks during the trials. For the analysis only trials without color change were used.

In the interview after Experiment 4.1.1 the question if the participant attended the second stimulus as well (described in Chapter 2) was altered to if they attended the stimuli at all. In the interview after Experiment 4.1.2 the question was added if they were distracted by the occasionally occurring color change of the fixation cross.

\subsubsection{EEG Recording}

EEG data were recorded using the BioSemi ActiveTwo recording system (BioSemi Inc. Amsterdam, The Netherlands) with $64 \mathrm{Ag} / \mathrm{AgCl}$ electrodes arranged on a standard BioSemi head cap according to the International 10-20 system. The $\mathrm{Cz}$ electrode was located midmost beteween the Nasion and the Inion. For monitoring the eye movements an electro-oculogram (EOG) was recorded. Horizontal eye movements (hEOG) were recorded by two monopolar electrodes placed on both external canthi. Vertical eye movements (vEOG) and blinks were recorded by a 
monopolar electrode and the FP1 electrode of the electrode cap placed below and above the left eye, respectively. Two monoploar reference electrodes were placed on both mastoids. EEG and EOG were sampled at $512 \mathrm{~Hz}$.

Participants sat in an armchair without chin rest in a separate recording room with all electrical appliances being switched off to avoid interferences. The experimental monitor was installed outside the recording room being oriented to an opening to the recording room. Participants were instructed not to blink during the trial to avoid EEG artifacts due to eye blinks. No auditory feedback was given to avoid interferences with visual ERPs.

\subsubsection{Data Analysis}

\section{Behavioral Data}

In Experiment 4.1.1 the Discrimination Sensitivity and the Response Bias was calculated for both SOAs as described in Chapter 2.4, for both, trials with and without color change of the fixation point averaged over all three possible time windows where the color change could occur. This data analysis served only for the observation of a proper task fulfillment and were not further considered.

The Experiment 4.2 was intended to be used for group categorization. When participants showed a Type A masking functions they would be assigned to "Group Type A" and when they showed a Type B masking function they would be assigned to "Group Type B". In contrast to the last three studies this distinct separation into two groups instead of a continuous approach was applied to realize a comparisons of the EEG data of two groups with distinct masking types. To implement an objective approach for categorization, the slopes of the masking functions were analyzed, using percent correct data as performance measurement. To set the upper and lower borders of the slopes, qualifying the assignment to one of both groups, the masking functions of the last four comparable Experiments (Experiments 1.1, 1.3, 2.1, and 3.1) were analyzed. In these experiments all datasets were manually categorized in either "Type A", or "Type B" masking functions when their course showed an obvious increasing (Type A) or decreasing (Type B) slope over SOAs, or in "Underachiever", when performance was very low in all SOAs showing no distinct "Type A" or "Type B" course, or in "Overachiever", when their performance was very high in all SOAs showing no distinct "Type A" or "Type B" course neither. It turned out that a slope of 1.5 or higher (Type A) and of -1.5 or lower (Type B) indicates a clear Type A and Type B masking function, respectively. All four overachievers, all but one of ten underachievers, five out of 31 Type A observers, and four out of 35 Type B observers are excluded for further analysis with these boundaries, which is judged as a good cost-benefit trade-off losing a small number of potential datasets to exclude most of the observers without clear type in return. 


\section{Study 4 - Effect of Attention}

In Experiment 4.2 the slopes for all masking functions were calculated, using only the second half of the trials, as it is assumed that masking function has established till then. Datasets with a slope of at least 1.5 were assigned to Type A and datasets with a slope of maximal -1.5 were assigned to Type B, provided that the masking course over SOAs was not arbitrary but resembles a typical Type A or Type B masking function. Participants whose slope was not within the parameters or whose masking function was untypical and could not be assigned to one group, although their slope was within the parameters, were not invited for the third session (Experiment 4.1.2) to go easy on resources. In retrospect, it would had been better to invite them, nevertheless, to have full datasets for possible additional analysis. This approach was implemented for the latter part of data acquisition. Next to the slope of the percent correct performance the Discrimination Sensitivity and the Mask Bias were analyzed to gain an overview over data distribution but they were not further considered.

In Experiment 4.1.2 the slopes over the two SOAs in the percent correct performance data were analyzed and participants were categorized according to the procedure described for Experiment 4.2. In addition, the Discrimination Sensitivity and the Mask Bias were analyzed to gain an overview over data. Comparing the masking type of Experiment 4.2 and Experiment 4.1.2. they were not identical for all participants. This result was surprising as it was expected to be constant over sessions. The change of type was undirected, it occurred from Type A to Type B and vice versa, from Type A or B to Overachiever, from Underachiever to Type A or B. It is hypothesized to find ERP differences between both groups. This hypothesis is based on different phenomenological perceptions reported by the participants and which are associated with the masking functions. To ensure that ERPs match to masking functions datasets were categorized after Experiment 4.1.2 instead of after Experiment 4.2.

\section{Physiological Data}

In Study 4 an electrophysiological measures were applied to combine behavioral results with physiological results. For this an electroencephalogram (EEG) was recorded.It is an extracranial measurement of electric potentials elicited by the electrical nerve cell activity of the brain. Event-related potentials (ERPs) are voltage changes in the continouous EEG, which are timelocked to external events, for example the onset of a stimulus, and are assumed to reflect the neural processing of this event (Coles \& Rugg, 1995). EEG have high temporal resolution for precise temporal outlines of neural processing. However, it has poor spatial resolution. Therefore, source localization is imprecise, electrode positions where ERPs were recorded may not be the origins of potentials and therefore give just a rough estimation of the involved brain areas. 
EEG data was analyzed with EEGLAB v.13.4.4b (Delorme \& Makeig, 2004) and ERPLAB version 5.0.0.0 (Lopez-Calderon \& Luck, 2014) in MATLAB R2013a (version 8.1.0.604) [Computer software]. For the hEOG recordings of right-sided electrode were subtracted from the recordings of left-sided electrode. For the vEOG recordings of the FP1 electrode were subtracted from recordings of the electrode below the eye. The continuous data were band-pass filtered $(0.01 \mathrm{~Hz}-30 \mathrm{~Hz})$ off-line, segmented in epochs from $210 \mathrm{~ms}$ before target-onset to $600 \mathrm{~ms}$ after target-onset, and corrected with a baseline from $200 \mathrm{~ms}$ to $0 \mathrm{~ms}$ before target-onset. Trials with artifacts were rejected before averaging. The threshold for artifacts rejection was in ocular electrodes $\pm 70 \mu V$, in scalp electrodes $\pm 120 \mu V$, for sample-to-sample-difference in all electrodes not more than $50 \mu V$, and in flatline-activity in scalp electrodes $\pm 1 \mu V$. Only datasets with more than 70 trials per condition were used, otherwise they were excluded. Major effects were expected at posterior electrodes. To localize cortical positions where stimulation effects were most pronounced topographical plots were analyzed. Here, time ranges around the components $\mathrm{P} 1$ and $\mathrm{N} 1$ of target and mask were considered. Based on these topographical plots further ERP analyses were conducted with particular electrode sites. Electrode sites of both hemispheres were averaged and analyzed as an electrode pair as it is assumed that EPRs are distributed equally over both hemispheres. ERPs were analyzed separately for congruent and incongruent trials as it is assumed that they are processed differently. The different processing of the condition with attention and without attention is of most interest. For better visualization, a difference curve was calculated where EEG waveforms of the condition without attention is subtracted from the condition with attention.

\section{Statistics}

Statistical analyses were conducted using R (R Core Team (version 3.0.1) [Computer software], 2013). ANOVAs were calculated to determine differences between groups, experimental sessions, and congruency. The analysis was conducted in the time range between 0 and $400 \mathrm{~ms}$ after target-onset in steps of $25 \mathrm{~ms}$, meaning that all datapoints were averaged within these $25 \mathrm{~ms}$ bin.

\subsection{Results}

\subsubsection{Behavioral Data}

Individual masking functions of Experiment 4.1.2 were categorized according to their slope. Twenty datasets were assigned to the group "Type A", their averaged performance is higher in the long SOA than in the short SOA (Figure 6.1a, page 76). Thirty-three datasets were assigned 
(a)
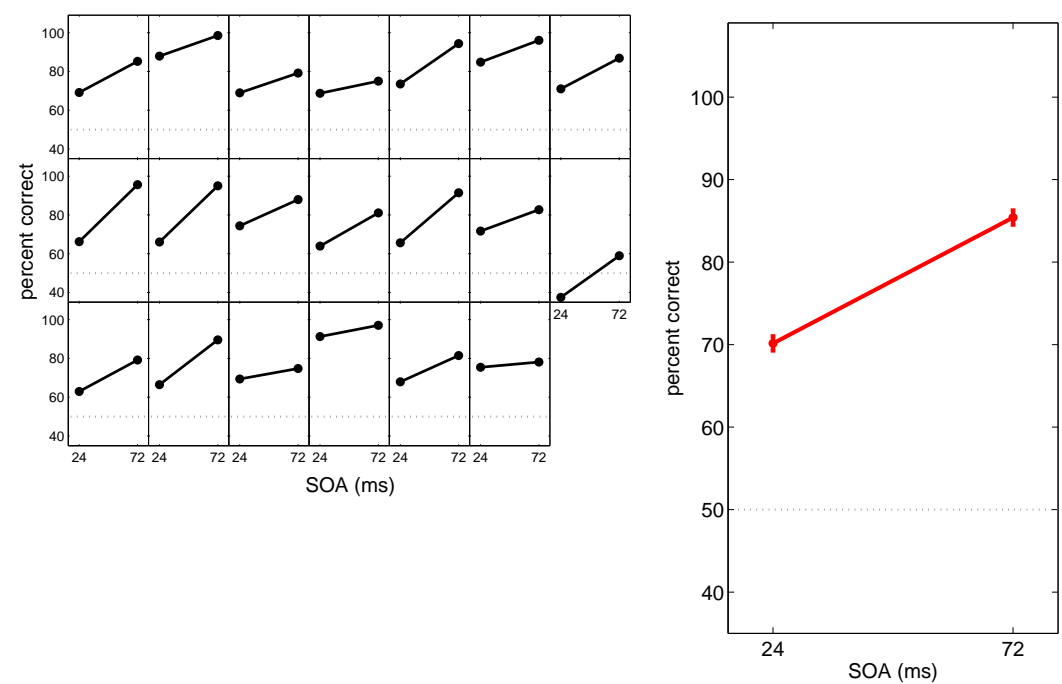

(b)
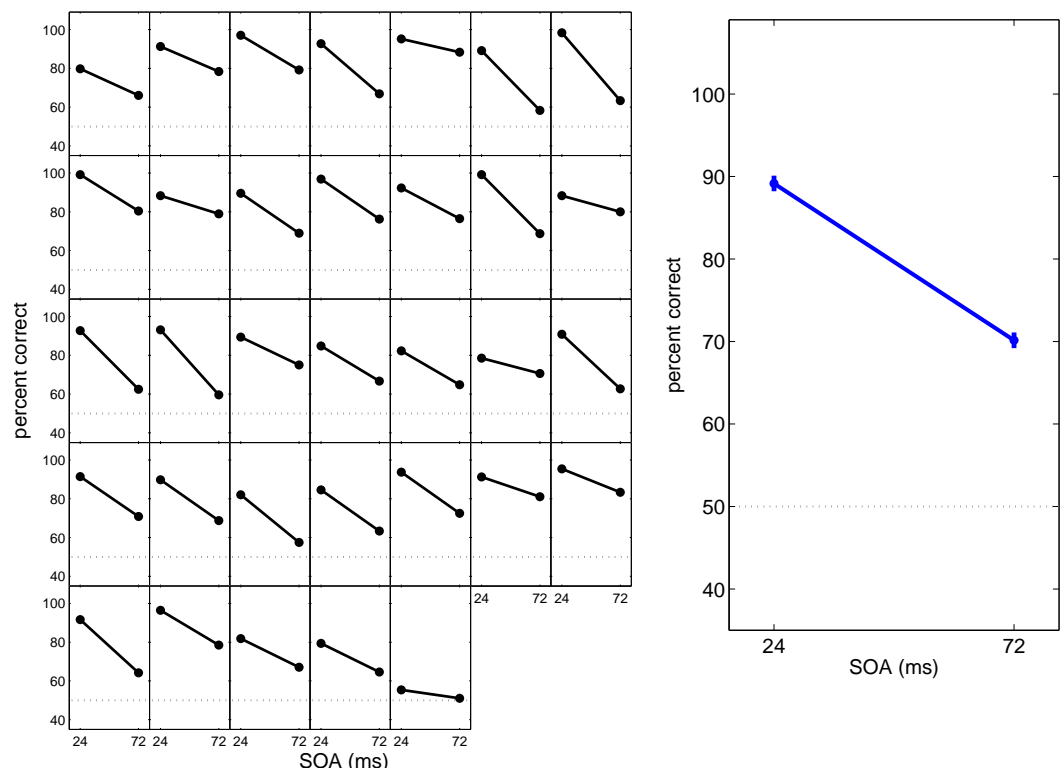

Figure 6.1: Experiment 4.1.2. Individual percent correct performance (left-hand side) and grand average (right-hand side) of datasets assigned to group (a) "Type A" and (b) "Type B". Error bars represent the within-subject standard error (Loftus \& Masson, 1994).

to the group "Type B", their averaged performance is higher in the short SOA than in the long SOA having a decreasing masking function (Figure 6.1b, page 76). The Sensitivity of the group "Type A" is increasing as well and the Mask Bias is decreasing over SOAs being positive in the short SOA; the Sensitivity of the group "Type B" is decreasing as well and the Mask Bias 
(a)

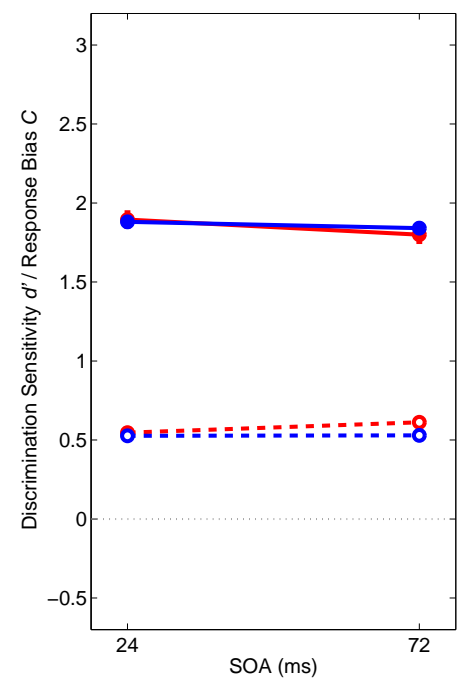

(b)

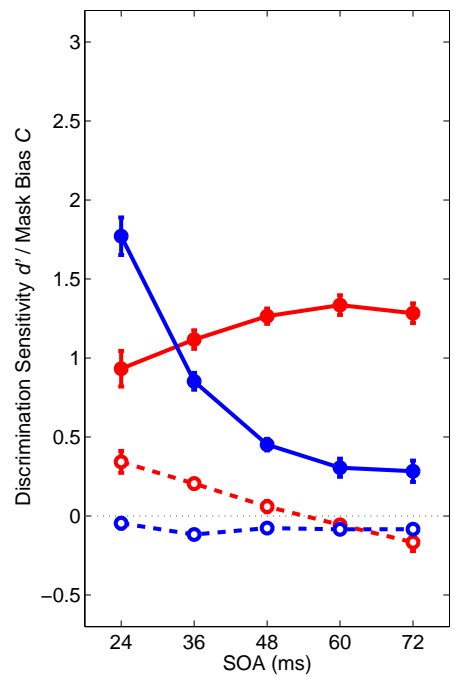

(c)

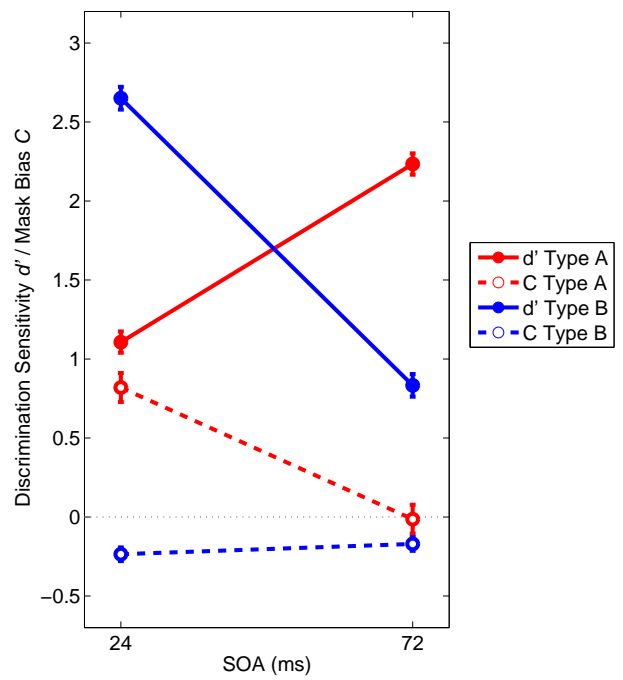

Figure 6.2: Discrimination Sensitivity $d^{\prime}$ and Response Bias $C_{R}$ and Mask Bias $C_{M}$, respectively, for groups Type A and Type B. (a) Experiment 4.1.1. (b) Experiment 4.2. (c) Experiment 4.1.2. Error bars represent the within-subject standard error (Loftus and Masson, 1994, note that some are too small to visualize).

is slightly negative and identical for both SOAs (Figure 6.2c, page 77) confirming the group assignment.

Statistical analysis confirm group differences depending on SOA (for Sensitivity two-way interaction Group x SOA: $F(1,51)=192.04, p<.001$; for Mask Bias two-way interaction Group x SOA: $F(1,51)=24.92, p<.001)$.

In Experiment 4.1.1 groups do not differ in their Sensitivity or their the Response Bias and there is no variation over SOAs (Figure 6.2a, page 77, for Sensitivity two-way interaction Group x SOA: $F(1,51)=0.27, p=.603$; for Response Bias two-way interaction Group x SOA: $F(1,51)=1.42, p=.239)$. As the three-way interaction Group x SOA x Experiment turned out to be significant, it can be stated that the groups differ in their masking function as well as in their Bias when fulfilling the target discrimination task but not when fulfilling the color detection task (for Sensitivity two-way interaction Group x SOA: $F(1,51)=178.82, p<.001$; for Bias two-way interaction Group x SOA: $F(1,51)=27.12, p<.001)$.

Discrimination Sensitivity and Mask Bias averaged for both groups in Experiment 4.2 are shown in Figure 6.2b (page 77). Although four participants (of 20) show a Type B and three (of 20) an ambiguous masking function, the averaged function has an Type A course increasing with increasing SOAs. The Mask Bias is positive in short SOAs and decreasing with increasing SOAs, which is typical for Type A. Similarly, two participants in group Type B had an increasing 


\section{Study 4 - Effect of Attention}

or ambiguous masking function. Nevertheless, the averaged function shows a typical Type B course decreasing with increasing SOAs. The Mask Bias is slightly negative and similar over SOAs, which is typical for Type B.

\subsubsection{Physiological Data}

Topographical plots were analyzed to localize cortical positions where stimulation effects were most pronounced. Based on Figure 6.3a (page 79), which shows the topographical distribution of the EPRs for the time range from $50 \mathrm{~ms}$ to $150 \mathrm{~ms}$ after target-onset in $10 \mathrm{~ms}$ steps, the electrode sites PO7/PO8 and O1/O2 were chosen for further ERP analysis, as the amplitudes were highest (see Figure 6.3b, page 79, for electrode site assignment).

Over all statistical tests only one showed a significant main effect between groups, the time range 25.1-50 ms of congruent trials with the SOA of $72 \mathrm{~ms}$ at the electrode pair O1/O2 $(F(1,51)=4.71, p=.039)$. As this is an isolated case it is disregarded. In contrast, significant interaction of groups with attention occurred (see statistical results in Tables 6.1 and 6.2, pages 84 and 85): For the PO7/PO8 electrode pair in the short SOA in congruent trials between 175 and $400 \mathrm{~ms}$, and in incongruent trials between 175 and $350 \mathrm{~ms}$; for the long SOA in congruent trials between 150 and $200 \mathrm{~ms}$ and between 250 and $400 \mathrm{~ms}$, and in incongruent trials between 250 and $400 \mathrm{~ms}$. For the O1/O2 electrode pair in the short SOA in congruent trials between 175 and $400 \mathrm{~ms}$, and in incongruent trials between 175 and $375 \mathrm{~ms}$; for the long SOA in congruent trials between 150 and $200 \mathrm{~ms}$ and between 250 and $400 \mathrm{~ms}$, and in incongruent trials between 225 and $400 \mathrm{~ms}$. Finding these interactions indicates a different top-down processing between Type A and Type B observers. These interactions can be visualized in the Figures 6.4-6.7 (pages 80-83) as follows: In the condition with attention (middle row) groups differ in their EEG deflections, beginning at a specific time point, while in the condition without attention (top row) there are only slight group differences. In the difference curve (bottom row) it can be seen if deflections are more positive or more negative in the condition with attention than without attention and if these changes are different for both groups. At PO7/PO8 in the short SOA the difference curve of the Type B is more negative than for the Type A group, meaning that EEG deflections are more negative/ less positive in the condition where attention was on metacontrast stimuli than when attention was not directed on them. Results are similar for congruent and incongruent trials. In the long SOA deflections for the Type A group are slightly more positive in the condition with attention while for the Type B group they are more negative. At $\mathrm{O} 1 / \mathrm{O} 2$ in the short SOA the same pattern as at PO7/PO8 can be observed. There are only small differences between conditions for the Type A group, but the Type B group became more negative. In the long SOA deflections for the Type A group are more positive in the condition with attention, while for the Type B group they are slightly more negative. 
(a)

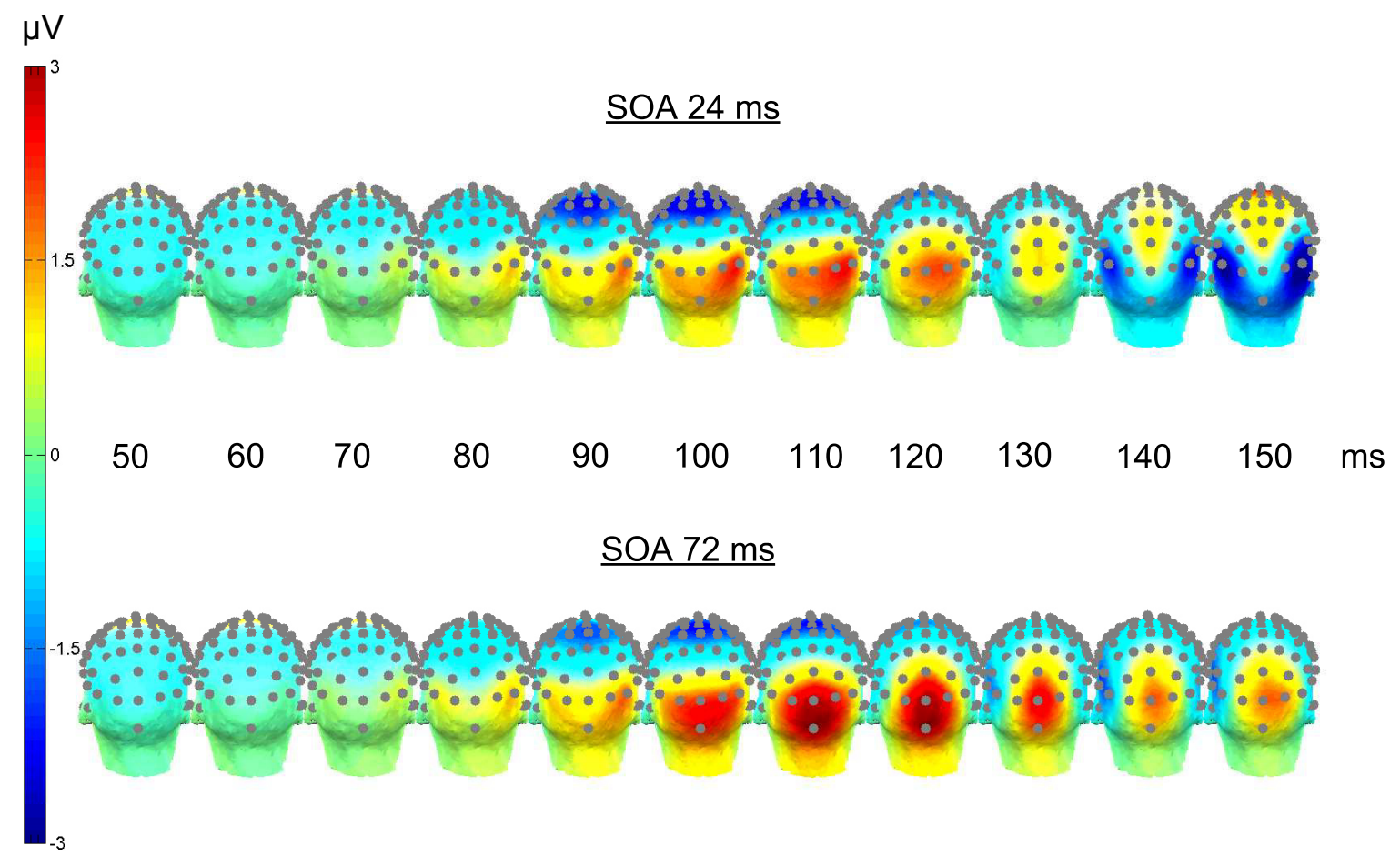

(b)

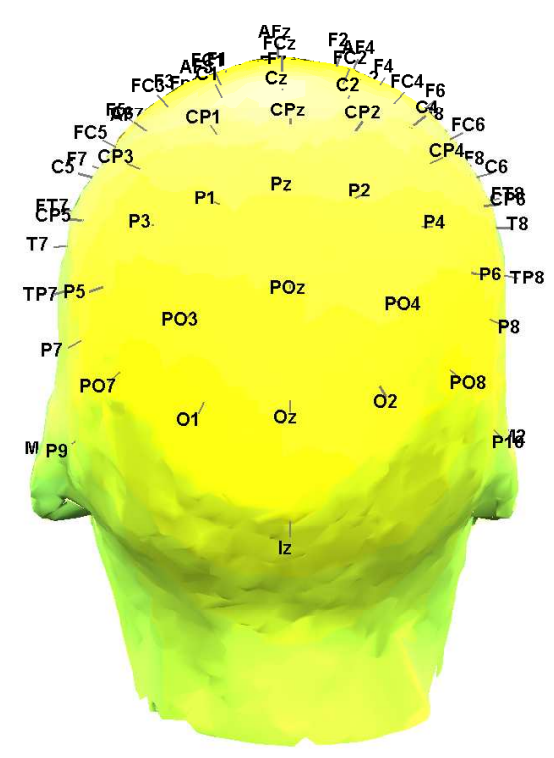

Figure 6.3: Experiment 4.1. (a) Topographical plots of the EPRs for the time range from $50 \mathrm{~ms}$ to $150 \mathrm{~ms}$ after target-onset in $10 \mathrm{~ms}$ steps. In the upper line trials with the SOA of $24 \mathrm{~ms}$ and in the lower line trials with the SOA of $72 \mathrm{~ms}$ are shown. In these plots participants of both groups, both sessions of Experiment, as well as congruent and incongruent trials are averaged. (b) Assigned electrode sites of the electrode cap. 


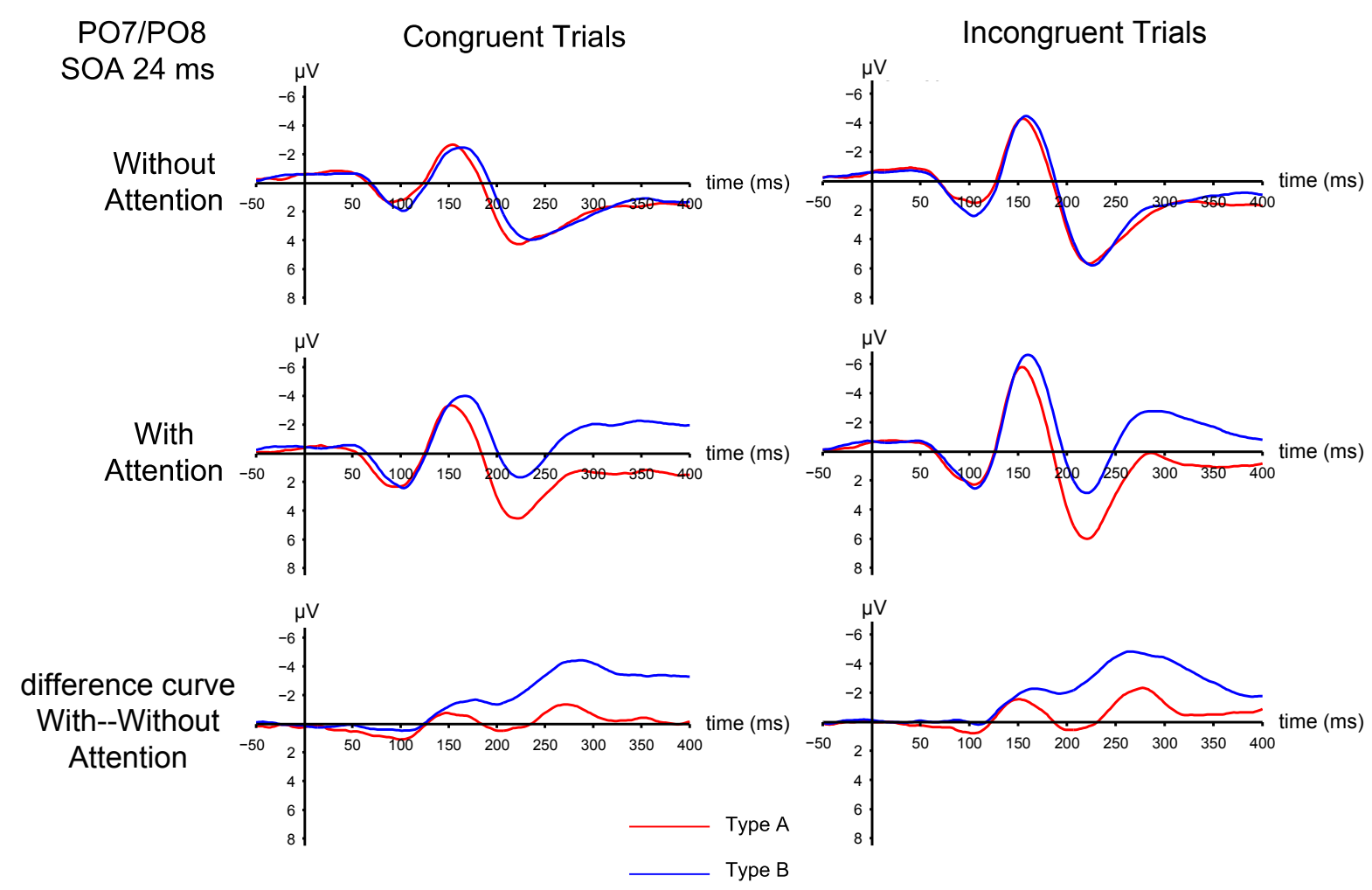

Figure 6.4: Experiment 4.1. ERPs waveforms at PO7/PO8 with 24 ms SOA. Upper row: condition without attention (Experiment 4.1.1), middle row: condition with attention (Experiment 4.1.2), bottom row: difference curve where condition without attention is subtracted from condition with attention. Left column: congruent trials, right column: incongruent trials. Negative values are plotted upwards.

\subsection{Discussion}

\subsubsection{Experimental Discussion}

In this experiment it was investigated how inter-individual differences are affected by different attention modulations to determine if these differences are based on different top-down processing modulated by different attentional mechanisms, or if they are based on different bottom-up processing, independent from attention. For a better comparison, individual datasets were classified into one of two groups representing Type A and Type B observers. It is assumed that, when finding a significant main effect for groups that processing differs bottom-up, and when an interaction of attention and group is found then processing differs top-down. Based on previous findings where Type A and Type B showed differences in their stimulus processing, it was examined if these group differences also occur when metacontrast stimuli were not attended. For this, two sessions were conducted with identical stimulus presentation. While in the first session metacontrast stimuli had to be ignored (without attention), in the second session they 


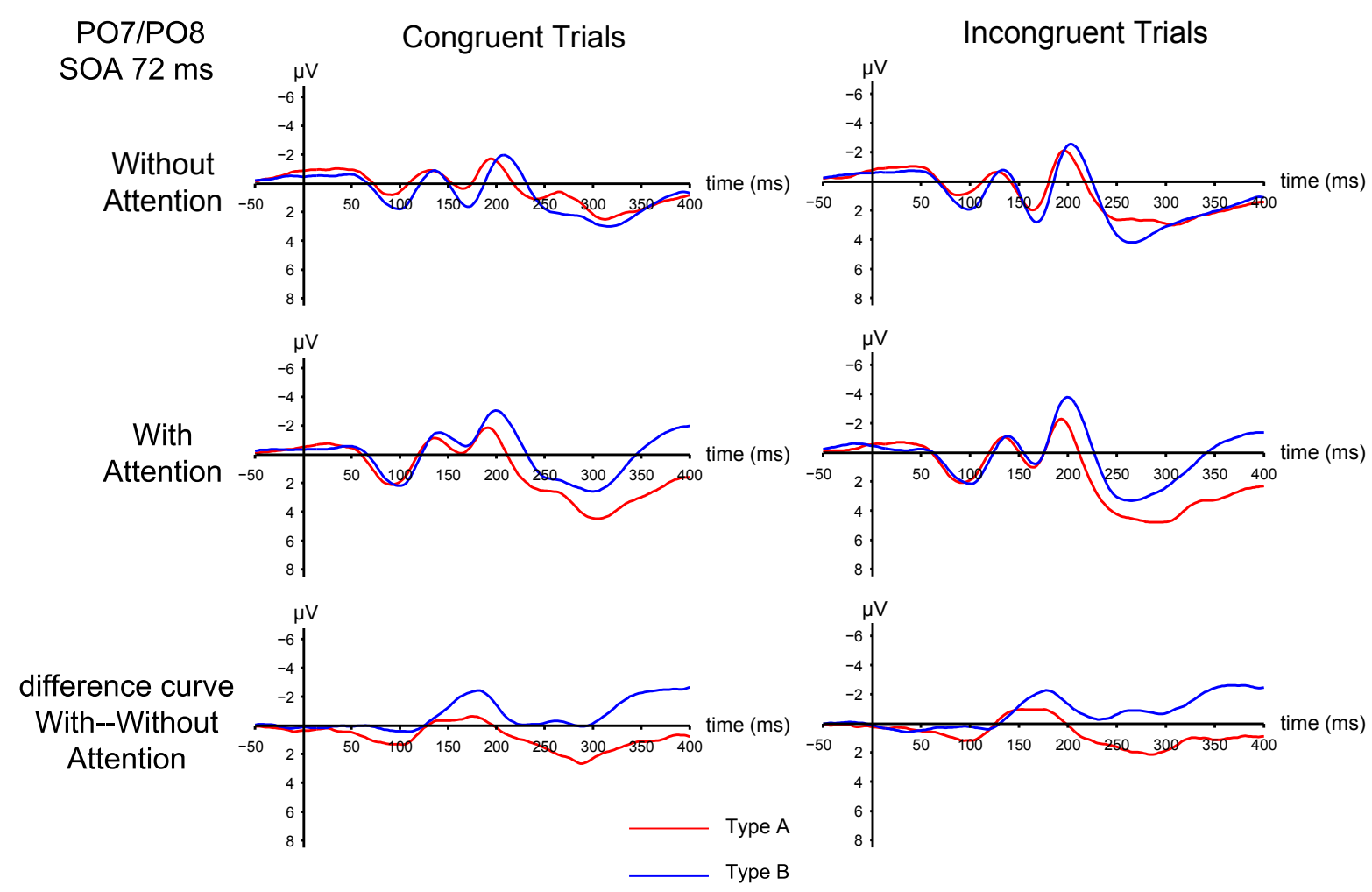

Figure 6.5: Experiment 4.1. ERPs waveforms at PO7/PO8 with 72 ms SOA. Upper row: condition without attention (Experiment 4.1.1), middle row: condition with attention (Experiment 4.1.2), bottom row: difference curve where condition without attention is subtracted from condition with attention. Left column: congruent trials, right column: incongruent trials. Negative values are plotted upwards.

had to be focused and the shape of the target had to be discriminated (with attention). Sensitivity and Response Bias do not differ between groups and SOAs in the condition without attention, but in the condition with attention masking functions and Mask Bias differ between groups.

It was further tested if these behavioral interactions of attention and group can also be found in the physiological data. No significant main effect of group was found indicating that groups do not differ only on bottom-up processes. However, strong significant interactions of attention and group were found between approximately $200 \mathrm{~ms}$ and $400 \mathrm{~ms}$ at the electrode sites $\mathrm{PO} 7 / \mathrm{PO} 8$ and $\mathrm{O} 1 / \mathrm{O} 2$ indicating that groups differ in their top-down processes modulated by attention. This attentional effect occurs in a relatively late time range, leaving the early components (P1and N1) unaffected. As the P1 component is more associated to spatial attention stimulus parameters (Handy, 2004) it further underlines the common bottom-up processing. However, Stojanoski and Niemeier (2011) found ERP modulation around the P1 component when attending to motion. Although Type A observers report to see and use apparent motion as cue for target discrimination, such modulations cannot be found in the present study. This may 


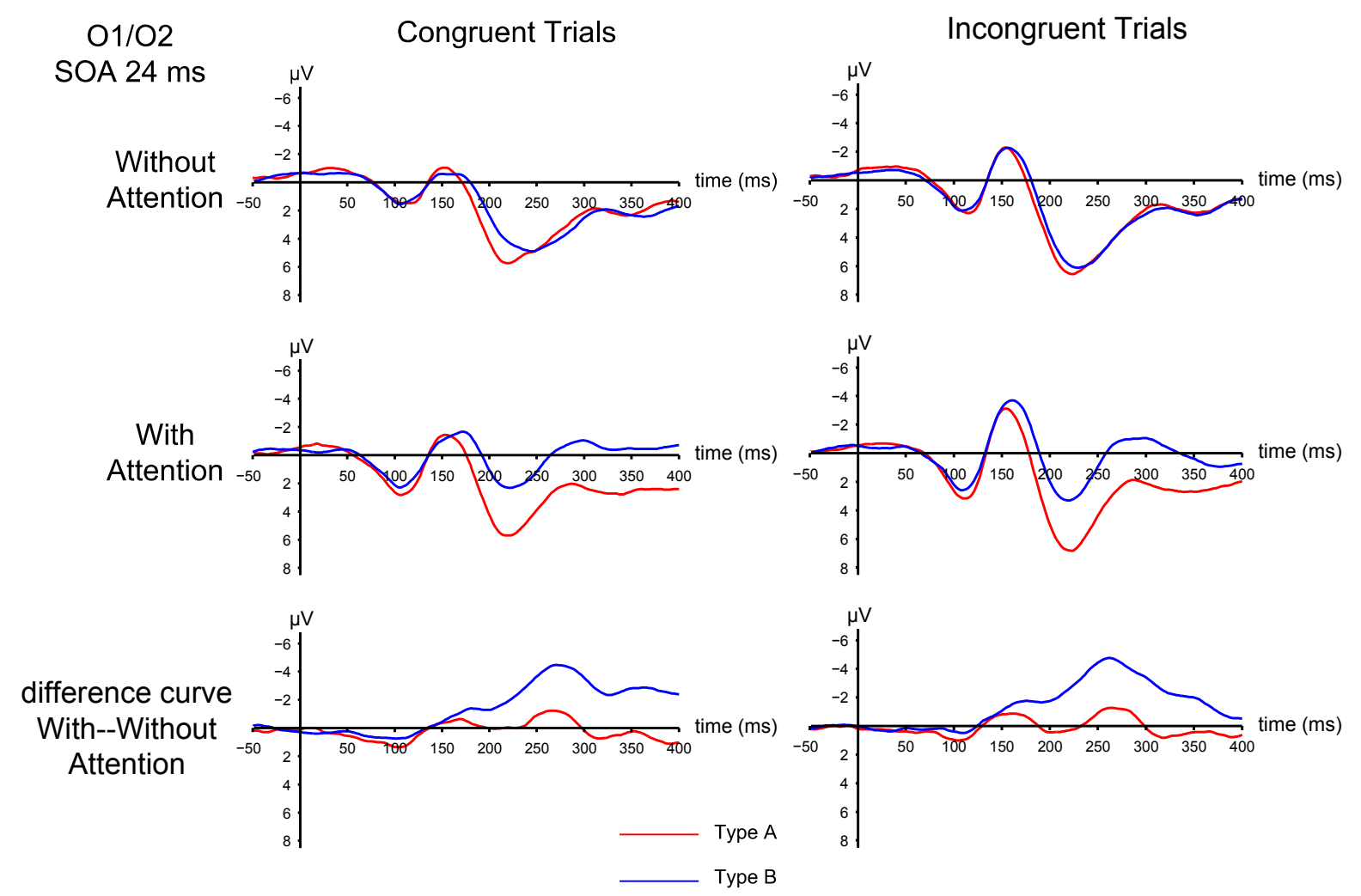

Figure 6.6: Experiment 4.1. ERPs waveforms at O1/O2 with 24 ms SOA. Upper row: condition without attention (Experiment 4.1.1), middle row: condition with attention (Experiment 4.1.2), bottom row: difference curve where condition without attention is subtracted from condition with attention. Left column: congruent trials, right column: incongruent trials. Negative values are plotted upwards.

indicate different kinds of motion perceptions processed differently. The N1 component is associated to discriminative processing being larger in discrimination tasks than in detection tasks (Handy, 2004). And in fact, at PO7/PO8 the ERPs are larger in the condition with attention where participants had to perform a target discrimination task. However, interactions just start becoming significant at this time, so the N1 component itself is not of significance for group differences. Later components are more difficult to assign to specific cognitive processes, partly because they may overlap and are not well isolated, but they are generally stronger related to higher cognitive functions. As group differences establish in this later time range, the underlying processes may be of higher cognitive nature. Further research is needed to specify the underlying top-down processes being specific for both groups. Stojanoski and Niemeier (2011) further report attention-based modulations in later phases around the P2 component (around $200 \mathrm{~ms}$ after target-onset) in association with motion detection and modulations in response to contours after the $\mathrm{P} 2$ component. In this phase, group differences begin to establish in the present study, which is an indication that group dependent different processing is based on dif- 


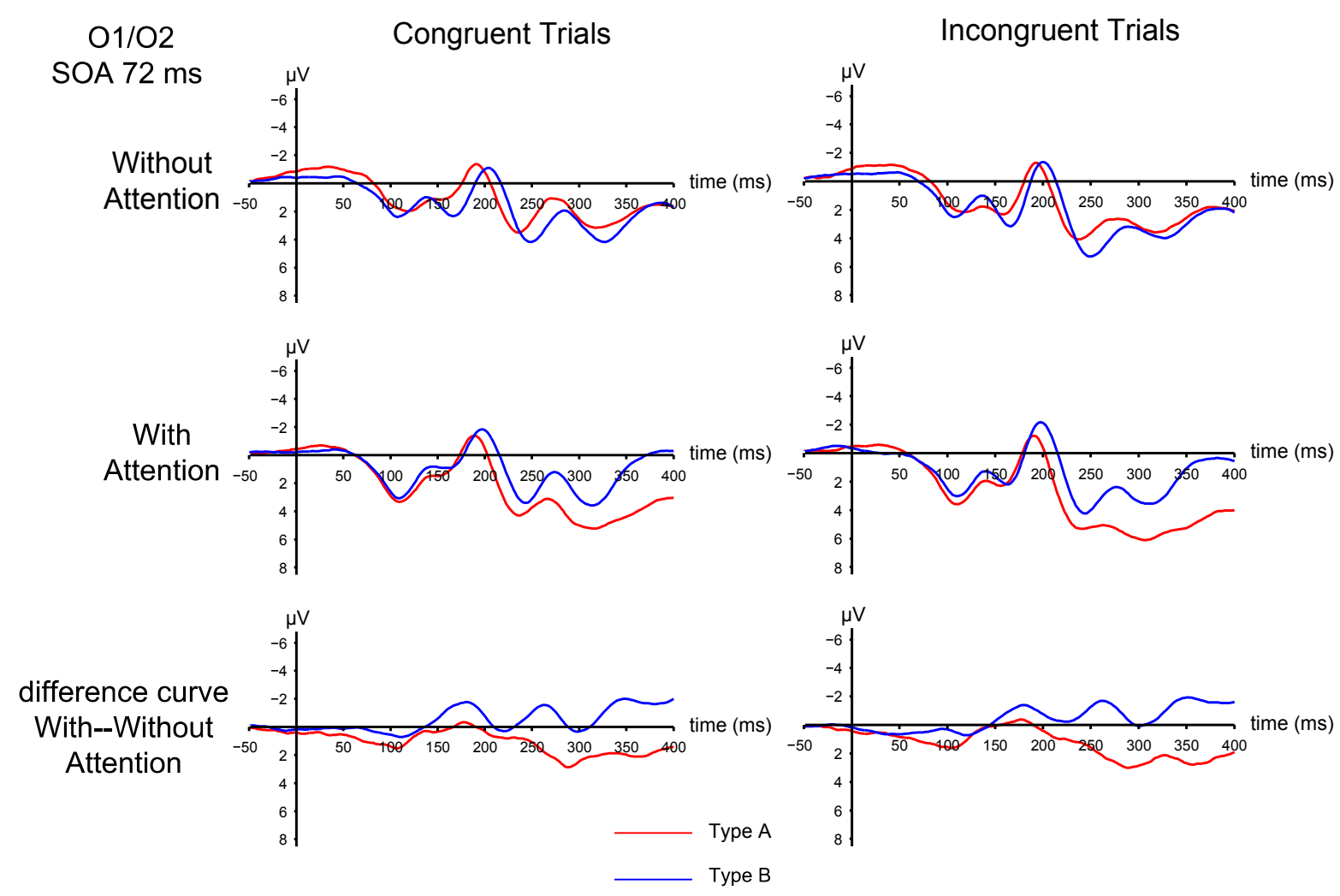

Figure 6.7: Experiment 4.1. ERPs waveforms at O1/O2 with 72 ms SOA. Upper row: condition without attention (Experiment 4.1.1), middle row: condition with attention (Experiment 4.1.2), bottom row: difference curve where condition without attention is subtracted from condition with attention. Left column: congruent trials, right column: incongruent trials. Negative values are plotted upwards.

ferent attention-related processing of motion and contours. Railo and Koivisto (2009) reported that more visible targets are associated with more negative (less positive) ERPs, than when targets are less visible in the time range of 330-420 ms after target-onset over the posterior temporal lobes (T5, T6). The ERPs of the Type B group are more negative in the condition with attention, than in the condition without attention beginning after $125 \mathrm{~ms}$ until $400 \mathrm{~ms}$. They are more pronounced and persevering in the short SOA than in the long SOA. As Type B observers report to see negative afterimages of the target in short SOAs but not in long SOAs these more negative ERPs might be associated to the better visibility of the target due to the afterimages. In contrast, Type A observers show more positive ERPs especially in the long SOA. There might be a comparable correlation of perceptions and strength of ERPs. Here again, further research is needed to approve this and investigate further correlations. 
Table 6.1: Experiment 4.1. Statistical results of the ANOVA at PO7/PO8 with $F(1,51)$ and $p$ values for all tested time ranges in ms ("time") after target onset. * $p<.05$.

\begin{tabular}{|c|c|c|c|c|c|c|c|c|c|c|c|c|}
\hline \multirow[b]{4}{*}{ time (ms) } & \multicolumn{12}{|c|}{$\mathrm{PO} 7 / \mathrm{PO} 8$} \\
\hline & \multicolumn{6}{|c|}{ SOA $24 \mathrm{~ms}$} & \multicolumn{6}{|c|}{ SOA $72 \mathrm{~ms}$} \\
\hline & \multicolumn{3}{|c|}{ congruent trials } & \multicolumn{3}{|c|}{ incongruent trials } & \multicolumn{3}{|c|}{ congruent trials } & \multicolumn{3}{|c|}{ incongruent trials } \\
\hline & $F$ & $p$ & & $F$ & $p$ & & $F$ & $p$ & & $F$ & $p$ & \\
\hline $0.0-25$ & 0.07 & .798 & & $<0.00$ & .982 & & 0.11 & .739 & & 0.21 & .132 & \\
\hline $25.1-50$ & 1.23 & .271 & & 0.58 & .452 & & 1.28 & .266 & & 0.16 & .695 & \\
\hline $50.1-75$ & 1.15 & .289 & & 1.49 & .227 & & 3.61 & .063 & & 0.46 & .500 & \\
\hline $75.1-100$ & 1.81 & .185 & & 2.22 & .142 & & 3.26 & .077 & & 2.22 & .142 & \\
\hline $100.1-125$ & 0.56 & .457 & & 0.84 & .365 & & 1.02 & .318 & & 0.30 & .589 & \\
\hline $125.1-150$ & 0.45 & .833 & & 0.05 & .818 & & 0.39 & .537 & & 0.08 & .782 & \\
\hline $150.1-175$ & 1.39 & .243 & & 1.22 & .175 & & 5.14 & .028 & $*$ & 1.76 & .190 & \\
\hline $175.1-200$ & 5.86 & .019 & $*$ & 6.69 & .013 & $*$ & 5.77 & .020 & $*$ & 2.91 & .094 & \\
\hline $200.1-225$ & 8.78 & .005 & $*$ & 14.06 & $<.001$ & $*$ & 1.56 & .218 & & 1.94 & .170 & \\
\hline $225.1-250$ & 11.54 & .001 & $*$ & 13.12 & $<.001$ & $*$ & 1.57 & .215 & & 3.68 & .061 & \\
\hline $250.1-275$ & 11.43 & .001 & $*$ & 8.81 & .005 & $*$ & 6.55 & .014 & $*$ & 13.16 & .001 & $*$ \\
\hline $275.1-300$ & 13.84 & $<.001$ & $*$ & 6.38 & .015 & $*$ & 8.37 & .006 & $*$ & 11.01 & .002 & $*$ \\
\hline $300.1-325$ & 12.22 & .001 & $*$ & 8.85 & .004 & $*$ & 5.88 & .019 & $*$ & 4.99 & .030 & $*$ \\
\hline $325.1-350$ & 8.61 & .005 & $*$ & 5.74 & .020 & $*$ & 8.58 & .005 & $*$ & 6.06 & .017 & $*$ \\
\hline $350.1-375$ & 10.39 & .002 & $*$ & 2.93 & .093 & & 11.51 & .001 & $*$ & 9.37 & .004 & $*$ \\
\hline $375.1-400$ & 9.71 & .003 & $*$ & 0.59 & .445 & & 8.02 & .007 & $*$ & 6.47 & .014 & $*$ \\
\hline
\end{tabular}

\subsubsection{Methodological Discussion}

The analyzed dataset has more Type B observers than Type A observers. Variations in the relation of Type B and Type A observers are normal as mostly only a small subset of the population is tested, however, this is a relatively large sample and both types were more balanced in the other studies, and therefore other factors may be responsible. An assumption is that the task of Experiment 4.1.1 influences the criterion content in the subsequent sessions. To be more precise, when performing the color-change detection task participants concentrate on the fixation point in the center of the monitor while metacontrast stimuli were presented. The criterion content of Type B observers involves the inner area of the mask as afterimages appear there. It may be that participants use in the detection task a comparable criterion content, which some kept in the second session, when they had to perform a form identification task (showing a Type B function), while others may realign their criterion content (and may show a Type A function). It was further observed that some participants had different masking functions in Experiment 4.2 and Experiment 4.1.2. This change refers to an inversion of the type, for example from Type B 


\subsection{Discussion}

Table 6.2: Experiment 4.1. Statistical results of the ANOVA at $\mathrm{O} 1 / \mathrm{O} 2$ with $F(1,51)$ and $p$ values for all tested time ranges in ms ("time") after target onset. * $p<.05$.

\begin{tabular}{|c|c|c|c|c|c|c|c|c|c|c|c|c|}
\hline \multirow[b]{4}{*}{ time (ms) } & \multicolumn{12}{|c|}{$\mathrm{O} 1 / \mathrm{O} 2$} \\
\hline & \multicolumn{6}{|c|}{$\mathrm{SOA} 24 \mathrm{~ms}$} & \multicolumn{6}{|c|}{ SOA $72 \mathrm{~ms}$} \\
\hline & \multicolumn{3}{|c|}{ congruent trials } & \multicolumn{3}{|c|}{ incongruent trials } & \multicolumn{3}{|c|}{ congruent trials } & \multicolumn{3}{|c|}{ incongruent trials } \\
\hline & $F$ & $p$ & & $F$ & $p$ & & $F$ & $p$ & & $F$ & $p$ & \\
\hline $0.0-25$ & 0.55 & .463 & & $<0.00$ & .971 & & 0.65 & .422 & & 0.02 & .898 & \\
\hline $25.1-50$ & 0.02 & .877 & & 0.09 & .762 & & 2.01 & .162 & & $<0.00$ & .985 & \\
\hline $50.1-75$ & 0.39 & .535 & & 0.62 & .434 & & 2.95 & .092 & & 0.82 & .370 & \\
\hline $75.1-100$ & 0.63 & .432 & & 0.88 & .352 & & 2.65 & .110 & & 3.17 & .081 & \\
\hline $100.1-125$ & 0.49 & .488 & & 0.56 & .458 & & 1.31 & .257 & & 1.15 & .289 & \\
\hline $125.1-150$ & $<0.00$ & .981 & & 0.13 & .722 & & 0.78 & .381 & & 0.01 & .917 & \\
\hline $150.1-175$ & 0.33 & .567 & & 1.30 & .259 & & 5.85 & .019 & $*$ & 1.75 & .191 & \\
\hline $175.1-200$ & 4.14 & .047 & $*$ & 7.09 & .010 & $*$ & 5.88 & .019 & $*$ & 2.71 & .106 & \\
\hline $200.1-225$ & 6.25 & .016 & $*$ & 13.24 & .001 & $*$ & 0.84 & .363 & & 2.00 & .164 & \\
\hline $225.1-250$ & 10.24 & .002 & $*$ & 12.54 & .001 & $*$ & 1.70 & .198 & & 4.98 & .030 & $*$ \\
\hline $250.1-275$ & 12.72 & .001 & $*$ & 14.43 & .004 & $*$ & 13.99 & $<.001$ & $*$ & 23.57 & $<.001$ & $*$ \\
\hline $275.1-300$ & 15.44 & $<.001$ & $*$ & 10.29 & .002 & $*$ & 12.24 & .001 & $*$ & 16.28 & $<.001$ & $*$ \\
\hline $300.1-325$ & 12.17 & .001 & $*$ & 9.65 & .003 & $*$ & 4.30 & .043 & $*$ & 6.21 & .016 & * \\
\hline $325.1-350$ & 6.97 & .011 & $*$ & 5.31 & .025 & $*$ & 10.84 & .002 & $*$ & 11.32 & .001 & $*$ \\
\hline $350.1-375$ & 12.52 & .001 & $*$ & 4.15 & .047 & $*$ & 18.98 & $<.001$ & $*$ & 17.13 & $<.001$ & $*$ \\
\hline $375.1-400$ & 13.64 & .001 & $*$ & 0.98 & .327 & & 10.61 & .002 & $*$ & 8.91 & .004 & $*$ \\
\hline
\end{tabular}

to Type A, and not to a variation of the same type. This outcome was unexpected as the only two variations between both experiments were the number of SOAs and the occasionally occurring color change of the fixation point. In 7 out of 53 cases the masking function changed from Type B or an unspecified type to Type A. It may be that the more time passes after Experiment 4.1.1 with the color-change detection task the more can the A process prevails and the criterion content is adapted. Only in 2 out of 53 cases the masking function changed from another type to Type B.

When not using a continuous classification like in the factor analysis, described in the last three studies, the classification of individual masking functions is often very subjective, especially when functions are not storybook. Therefore it is aimed to find an objective approach for distinct classification. Using the slope of the regression line over all SOAs was such an attempt. Unfortunately, it turned out not to be good enough. One reason may be that the selected boundaries are not appropriate, although carefully evaluated, and it will be difficult to find better assumptions for specification. Another reason why the selected criteria did not work 


\section{Study 4 - Effect of Attention}

out may be the fact that participants changed their type. When using the slope for classification another point has to be considered: First, when using long SOAs the U-shaped masking function may already increase again, which impairs the slope. A similar problem may occur for Type A masking functions, in long SOAs they may show a ceiling effect or an decrease of visibility.

\subsection{Conclusion}

This study aimed to investigate if inter-individual differences, which occur when using specific shapes of metacontrast masking stimuli, are bottom-up driven regarding basic stimulus processing, or if they are top-down driven so that processing can be modulated by attention. It turned out that inter-individual differences occur due to different top-down processes. When not attending the stimuli, they are processed similar by both groups of Type A and Type B observers. But when attention is on them, both groups show differences in their processing starting approximately $200 \mathrm{~ms}$ after target-onset. Further research is needed to specify the underlying top-down processes being specific for both groups. 


\section{Study 5 - Effect of the Instructed Task}

Since the beginning of metacontrast masking research it was associated with perception of brightness and contours (see Breitmeyer \& Öğmen, 2006, Chapter 1 for a historical background). As in masking visibility is reduced only some features of the target are processed leading to percepts different from the physical stimulus parameters. In numerous studies about brightness perception (e.g. Growney, 1976; Paradiso \& Nakayama, 1991; Petry, 1978), contour perceptions (e.g. Breitmeyer \& Tapia, 2011; Cox, Dember, \& Sherrick, 1969; Growney, 1976; Kolers, 1962), and apparent movement perception (e.g. Ansorge, Breitmeyer, \& Becker, 2007; Kahneman, 1967; Ramachandran \& Cobb, 1995) it was attempted to got to the bottom of metacontrast masking. Those different perceptions are interesting as they allow to specify which processing structures are involved in metacontrast masking. While the intensity or even reversal of brightness is mostly a phenomenological percept, contours are essential for the masking effect. The less the mask surrounds the target and the more distant the contours of both stimuli are, the weaker the masking effect is (e.g. Kolers, 1962). Another phenomenological percept is the perception of apparent movement, either expanding or rotating (e.g. Didner \& Sperling, 1980; Ramachandran \& Cobb, 1995; Stoper \& Banffy, 1977). Kahneman (1967) once postulated that metacontrast masking and movement perception may underly the same processes but this was soon withdrawn by Weisstein and Growney (1969). However, apparent movement and metacontrast masking are often associated (e.g. Stoper \& Banffy, 1977).

In their study from 2006 Breitmeyer et al. examined the differences between contour and brightness processing. Based on the assumption that different criterion contents effect the masking functions, participants made two different tasks with disc and ring stimuli. In the brightness judgment task the contrast of two stimuli had to be compared, while in the contour discrimination task it had to be detected where the contour deletion of the target was located. They found that the minimum of the U-shaped masking function is at lower SOAs for the contour task than for the brightness task indicating that contours are processed more early than surfaces.

In the priming study by Ansorge, Becker, and Breitmeyer (2008) different measures with different tasks were compared. They used square and diamond shaped primes and targets, one group had to fulfill a shape detection task, where participants had to respond with yes when the shapes of prime and target were identical, and another group had to fulfill a rotation detection task, where they had to respond with yes when prime and target had different shapes. While in 


\section{Study 5 - Effect of the Instructed Task}

the shape detection task performance did not differ between congruent and incongruent trials, in the rotation detection task performance was better than chance in incongruent trials but not in congruent trials

This study aims to determine how inter-individual differences depend on the instructed task to fulfill in a metacontrast masking experiment and if individuals process different aspects of stimulus information differently. As in the Studies 1 to 3, inter-individual differences were used to get information about the characteristics of the underlying processes. The study is composed of two metacontrast experiments: In Experiment 5.1 the SOA is varied and participants had to discriminate the shape of the target. It is expected to obtain individually different masking functions for each participant, which can be used for the factor analysis, whose factors describe the individual differences and represent the underlying processes. In Experiment 5.2 participants fulfill two different tasks on identical visual stimulation, one where contours of the target have to be processed and another task where the surface of the target has to be processed, referring to studies of Breitmeyer et al. (2006) and Ansorge et al. (2008). It is expected that masking functions of Type A observers are in the contour task comparable to those in Experiment 5.1, indicating the relevance of contour processing for those individuals, as Type A observers typically rely on contour information to detect apparent movements. In contrast, it is expected that masking functions of Type B observers are in the surface task comparable to those in Experiment 5.1, indicating the relevance of surface processing for those individuals, as Type B observers typically rely on the surface information in the form of negative afterimages. Furthermore, the individual factor scores of the factor analysis of Experiment 5.1 were correlated with the Discrimination Sensitivity and the Response Bias of Experiment 5.2 to obtain a relation measure, which provide information about the contribution of the specific process on the individual masking function.

\subsection{Methods}

\subsubsection{Participants}

Thirty-one healthy participants (10 male) participated in this experiment. Data of one female participant had to be excluded for analysis as she misunderstood the task. All but five were right-handed, and their mean age was $\mathrm{M}=23.7$ ( $\mathrm{SD}=3.1$ ), ranging from 19 to 30 .

\subsubsection{Tasks}

In Experiment 5.1 participants had to identify the shape of the target by pressing the left control key on the keyboard to answer that it was a square, and by pressing the right control key to 
answer that it was a diamond. In one session of Experiment 5.2 participants fulfilled a task where the contours of the target had to be judged (Experiment 5.2.1). They had to report if they saw a rotating movement in the sequence of target and mask by pressing the left control key on the keyboard to answer that it they did not see any rotation, and by pressing the right control key to answer that they did see a rotation. The identification of the target was irrelevant. In the other session the surface of the target had to be judged (Experiment 5.2.2). Here, participants had to report how clearly they saw the target without identifying the shape of the target. For they used the Perception-Awareness-Scale (PAS) by Ramsøy and Overgaard (2004) with four possible answer categories: "1" means "not seen at all", "4" means "clearly seen", and "2" and "3" were intermediate perceptions. The order of both sessions were randomized and balanced over all participants. Their response was not pressed for time in any session.

\subsubsection{Experimental Set-Up}

The study is composed of two experiments with three sessions in total. Stimuli were presented as shown in Figure 2.1b (page 10) in all sessions. In Experiment 5.1864 trials were presented in 12 blocks with 144 repetitions per SOA. In both sessions of Experiment 5.2672 trials were presented in 12 blocks with 112 repetitions per SOA. In addition, 96 mask-only trials were presented showing a fixation cross instead of the target stimulus.

The interviews after each session differ between the tasks. After Experiment 5.1 questions as described in Section 2.3 were asked including the additional questions about afterimages and movements. After Experiment 5.2.1 participants were asked how difficult they judged the task, how they solved the task (and if they had strategies they used), if there were trials where they perceived a rotation (and if yes, how often and how clear), if they had a perception different from a rotation in the sequence of both stimuli, how well they managed to fixate the fixation cross, if they were motivated over the whole session to do the task as best as possible, if they were tired, and if they had any problems. After Experiment 5.2.2 participants were asked how difficult they judged the task, how they assigned the four scale point to their perceptions, to judge the number of block after which their assignment was constant, if they put their attention on the shapes of the stimuli, and if yes, if the shapes were part of the assignment, how well they managed to fixate the fixation cross, if they were motivated over the whole session to do the task as best as possible, if they were tired, and if they had any problems during the session.

\subsubsection{Data Analysis}

For Experiment 5.1 the Discrimination Sensitivity $d^{\prime}$, the Mask Bias $C_{M}$, and the Factor Analysis were calculated as described in Chapter 2.4. In Experiment 5.2 Discrimination Sensitivity $d^{\prime}$ 


\section{Study 5 - Effect of the Instructed Task}

and the Response Bias $C_{R}$ were calculated using the signal detection theory by Macmillan and Creelman (2005). For Experiment 5.2.1 the proportion of "rotation yes" reports in incongruent stimuli were defined as hits, and "rotation yes" reports to congruent trials were defined as false alarms. This was done for each SOA and separately for each mask shape and then averaged over both shapes. In addition, the proportion of "rotation yes" reports in mask-only trials were reported, again first separately for both mask shapes and then averaged over both shapes. For Experiment 5.2.2 hits and false alarms were calculated with three different assignments of the reports. In the end, these three calculations were averaged and the average was used for further analysis. In the first calculation the proportion of " 4 " reports in trials with target and mask were defined as hits, and "4" reports in mask-only trials were defined as false alarms. In the second caluclation, the proportion of " 4 " as well as " 3 " reports in trials with target and mask were defined as hits, and " 4 " as well as " 3 " reports in mask-only trials were defined as false alarms. And in the third calculation the proportion of "4", "3" as well as "2" reports in trials with target and mask were defined as hits, and "4", "3" as well as "2" reports in mask-only trials were defined as false alarms. All calculations were done for each SOA and separately for each mask shape and then averaged over both shapes.

In both experiments analysis of variances (ANOVA) were conducted to examine the overall effects of SOA. To test for the effect of individual differences in Experiment 5.2, a full factorial repeated measures analysis of covariance (ANCOVA) was conducted with the within-subject factor SOA, and the two continuous between-subject covariates Type A Score and Type B Score obtained in Experiment 5.1. ANCOVA models included all pure within-subject effects, the main effect for each covariate, and the interactions of each covariate with all within-subject effects. Thus, all effects including an interaction of both covariates were excluded. The slope of the regression of $d^{\prime}$ and $C_{R}$ values with the factor scores, the slope parameter $\beta$, is calculated for each SOA, representing the correlation between $d^{\prime}$ and $C_{R}$ values with the factor scores. Statistical analysis was conducted using R (R Core Team (version 3.0.1) [Computer software], 2013).

\subsection{Results}

\subsubsection{Experiment 5.1}

\section{Discrimination Sensitivity and Mask Bias}

The individual masking functions and mask biases with the grand averages are shown in Figure 7.1 (page 91). Similar to Study 1 to 4 there is substantial inter-individual variability with regard to the slopes and the levels. The Discrimination Sensitivity over all datasets are inconclusive without variability over SOAs (main effect SOA: $F(5,145)=1.72, p=.196$ ). In contrast, the 


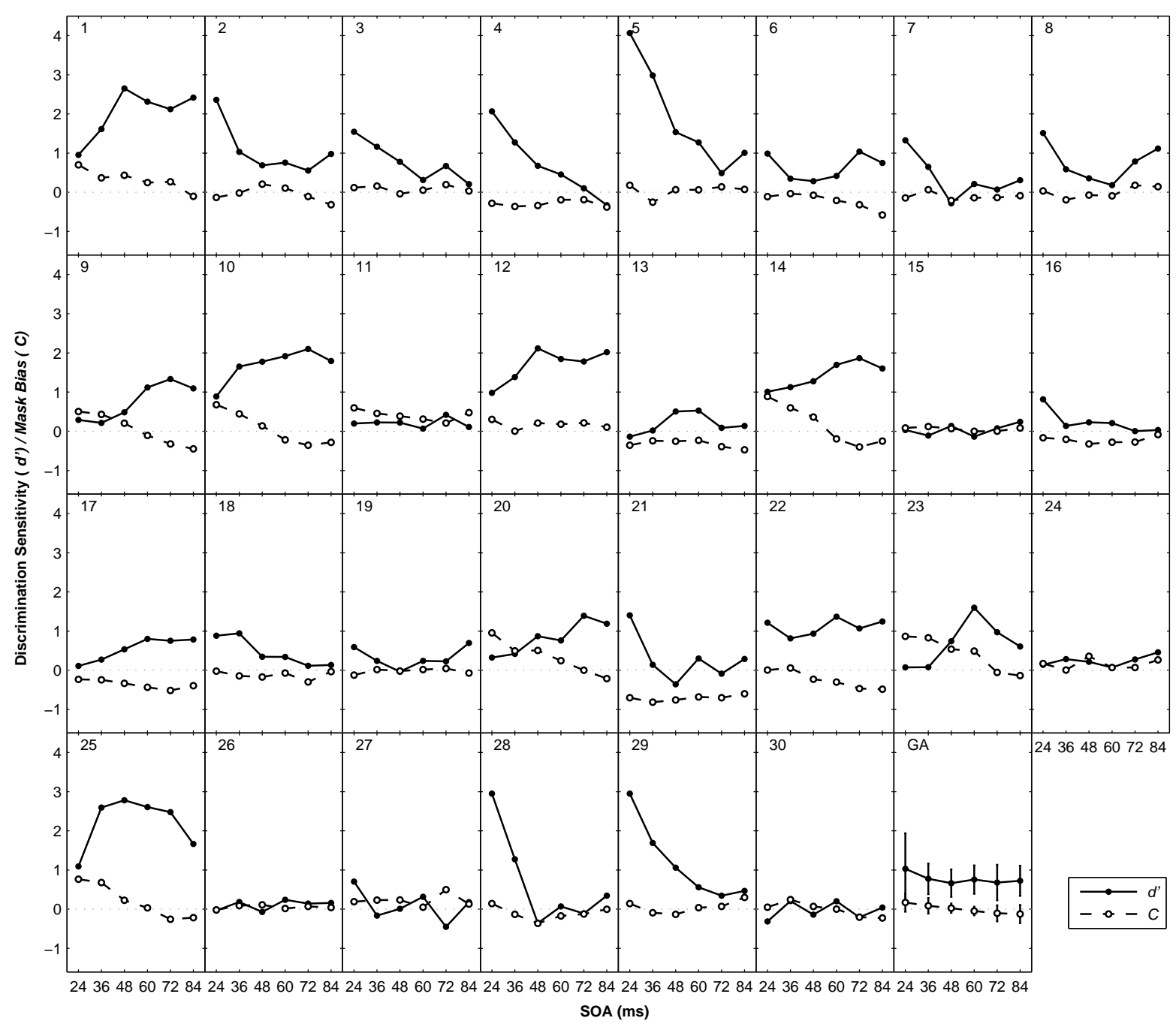

Figure 7.1: Experiment 5.1. Discrimination Sensitivity $d^{\prime}(\bullet)$ and Mask Bias $C_{M}$ (०) (individual plots [1-30] and grand average [GA]). Error bars represent the within-subject standard error (Loftus \& Masson, 1994).

Mask Bias $C_{M}$ of the grand average differs significantly over SOAs with slightly positive values in short SOAs and slightly negative values in long SOAs (main effect SOA: $F(5,145)=9.156$, $p=.001)$.

\section{Factor Analysis}

The maximum likelihood factor analysis revealed two factors with eigenvalues $>1$, which together explain $88 \%$ of total variance. However, the test shows that two factors are not sufficient $\left(\chi^{2}(4)=10.73, p=.030\right.$, see Table 7.1 , page 93). The maximum likelihood factor analysis with three factors revealed two factors with eigenvalues $>1$ and one factor with an eigenvalue $>1$, which together explain $94 \%$ of total variance. The degrees of freedom for the model is 0 


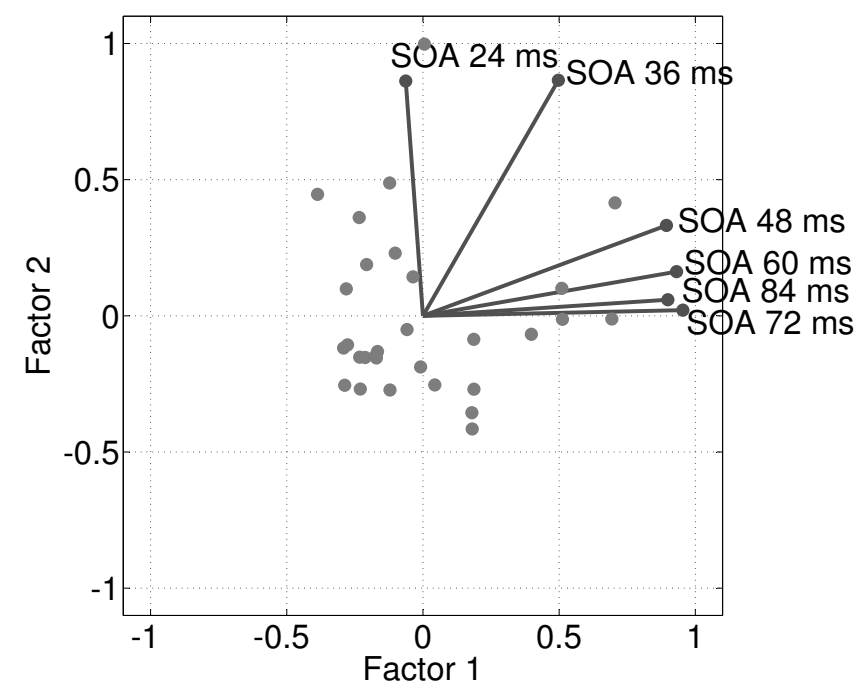

Figure 7.2: Experiment 5.1. Factor analysis - biplot with factor loadings (-) for each SOA and factor scores for each participant $(\bullet)$. (Please note that scales refer to factor loadings.)

and the fit is $p=.025$. Although with three factors more variance is explained, this third factor does explain less than one variable (one SOA). Therefore, the two-factor-solution is preferred. Loadings of Factor 1 are increasing over SOAs, while loadings of Factor 2 are decreasing (Figure 7.2, page 92). As the results are in line with Study 1 to 3 and the assumptions are the same, Factor 1 and 2 are named "Factor A" and "Factor B", respectively. Figure 7.2 also shows also the factor scores for each participant. Here again, individuals have high or low scores on both factors.

\section{Correlation of Mask Bias With Individual Factor Scores}

To investigate a possible relation of Mask Bias $C_{M}$ with individual masking performance an ANCOVA was conducted with SOA as repeated-measures independent variable and the individual factor scores as covariates. For visualization, the slope parameter $\beta$ is shown in Figure 7.3 (page 93) representing how strong individual factor scores correlate with $C_{M}$ values. Factor $\mathrm{A}$ is positive in short SOAs and decreasing with increasing SOA. As this interaction turned out to be significant (two-way interaction Factor A scores x SOA: $F(5,135)=18.50, p<.001$ ), it can be stated that higher Factor A scores lead reliably to a greater Mask Bias values in short SOAs, but are not predictive about the Mask Bias in long SOAs. The impact of Factor B is slightly negative in short SOAs and slightly positive in long SOAs. Although this impact is rather small the ANCOVA turned out to be significant (two-way interaction Factor B scores $\mathrm{x}$ SOA: $F(5,1358)=3.84, p=.003)$ implicating that the Factor B scores is of relevance for the manifestation of the Mask Bias. 
Table 7.1: Experiment 5.1. Factor analysis with two factors -- factor loadings for each SOA.

\begin{tabular}{rccc}
\hline SOA & Factor 1 & Factor 2 & communalities \\
\hline $24 \mathrm{~ms}$ & -0.063 & 0.862 & 0.747 \\
$36 \mathrm{~ms}$ & 0.497 & 0.865 & 0.995 \\
$48 \mathrm{~ms}$ & 0.894 & 0.332 & 0.910 \\
$60 \mathrm{~ms}$ & 0.931 & 0.162 & 0.893 \\
$72 \mathrm{~ms}$ & 0.954 & 0.021 & 0.911 \\
$84 \mathrm{~ms}$ & 0.899 & 0.059 & 0.811 \\
\hline sum of squares & 3.635 & 1.631 & \\
proportion variance & 0.606 & 0.272 & \\
cumulative variance & 0.606 & 0.878 & \\
\hline
\end{tabular}

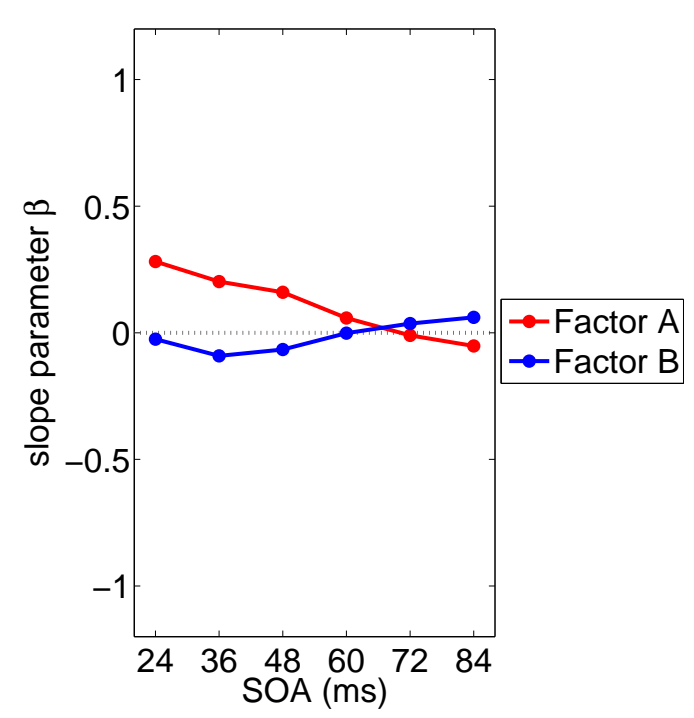

Figure 7.3: Experiment 5.1. Slope parameter $\beta$ over SOAs, with Mask Bias $C_{M}$ values correlated with Factor A scores and Factor B scores.

\subsubsection{Experiment 5.2}

\section{Experiment 5.2.1 Contour Task}

Discrimination Sensitivity. In the contour task participants had to report if they saw a rotation in the sequence of target and mask or not. The SOA was varied and the stimulus configuration enables congruent and incongruent tasks. It is expected that rotations were perceived in incongruent trials (if at all). The higher the Sensitivity $d^{\prime}$ the better can be discriminated between congruent and incongruent trials and therefore it can be reliably discriminated between rotation in incongruent trials and no rotation in congruent trials. The mask-only trials served as controls as no apparent rotation could be perceived. Only two participants answered to see 


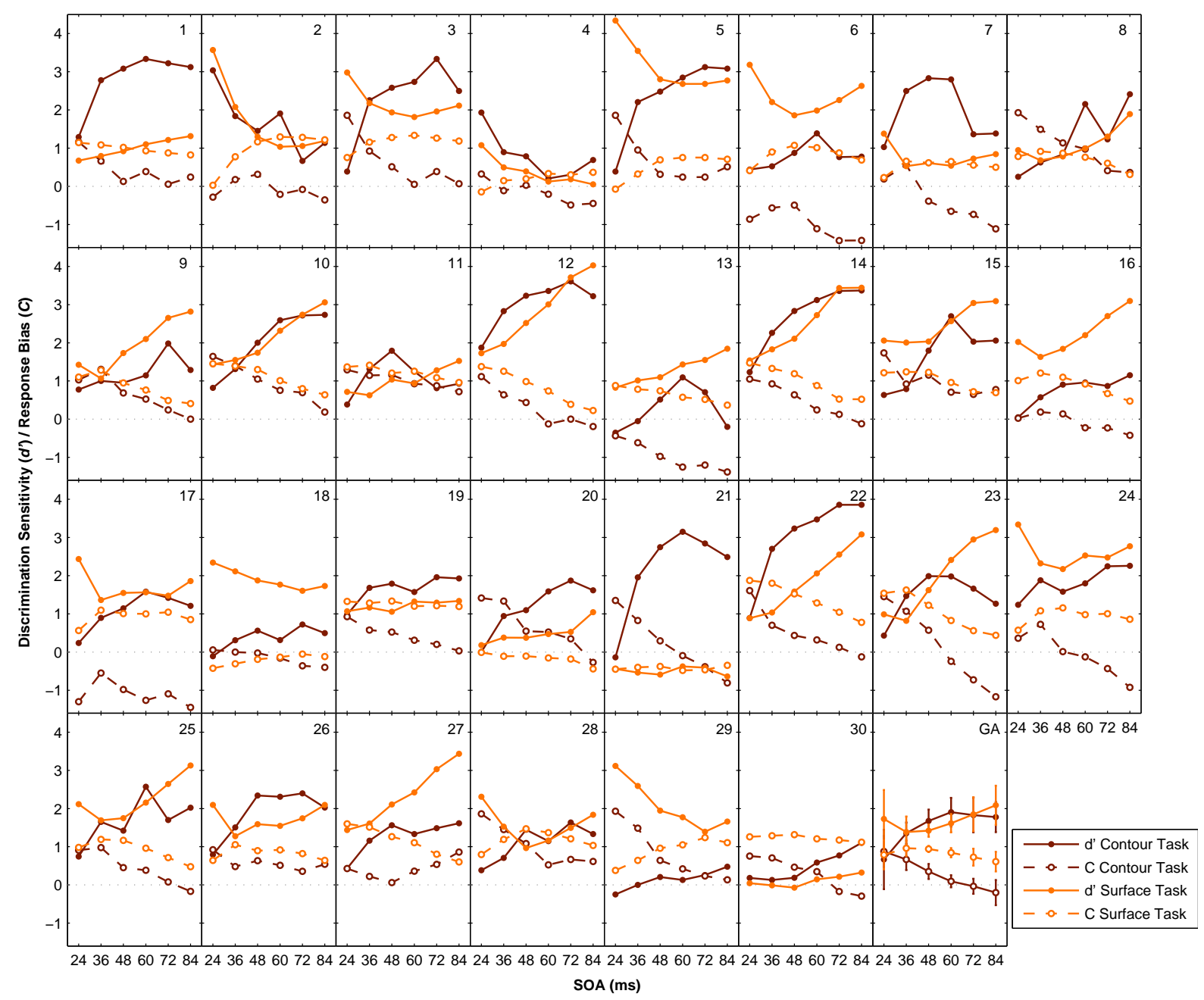

Figure 7.4: Experiment 5.1. Masking functions $d^{\prime}$ and Response Bias $C_{R}$ of the contour task and the surface task (individual plots [1-30] and grand average [GA]). Error bars represent the within-subject standard error (Loftus \& Masson, 1994).

a rotation in more than $40 \%$ of the mask-only trials. There are inter-individual differences in Sensitivity (Figure 7.4, page 94). Some participants have a low $d^{\prime}$ in short SOAs and increasing with increasing SOA (e.g. number 1, 12, or 22). Others have comparable low $d^{\prime}$ over all SOAs (e.g. number 6,18 , or 29) indicating that those have more difficulties to detect rotations. Only two participants (number 2 and 4 ) show a high $d^{\prime}$ in short SOAs but a low $d^{\prime}$ in long SOAs, which is rather unusual as it is expected that rotations can be perceived the better the longer the SOA is. The Sensitivity of the grand average is increasing with increasing SOA (main effect SOA: $F(5,145)=25.55, p<.001)$. Therefore, in general, rotations can be better perceived the longer the SOA is. 
Response Bias. The Response Bias $C_{R}$ reflects the tendency to answer for or against a rotation. Positive values reflect the bias to answer that they perceived no rotation (conservative response) and negative values reflect the bias to answer they perceived a rotation (liberal response). Most of the participants have a high Bias to answer that they perceived no rotation in short SOAs (Figure 7.4, page 94). With increasing SOA this Bias decreases to around zero in some participants (e.g. number 14, or 19, or 29) and in others to negative values, indicating the bias to answer that they perceived a rotation (e.g. 21, 23, or 30). Still others show a negative Bias (e.g. 6, 13, or 17) even in short SOAs although it further decreases in long SOAs. The overall Response Bias is positive in short SOAs and decreasing with increasing SOA becoming slightly negative in the longest SOA main effect SOA: $F(5,145)=57.11, p<.001)$.

\section{Experiment 5.2.2 Surface Task}

Discrimination Sensitivity. In the surface task participants had to report how clear if at all they saw the target. This judgment was made with a four-steps scale ranging between "1 - not seen at all" and "4 - clearly seen". The average of the three hit calculation resembles mostly the second calculation, where "4" and " 3 " reports in target-and-mask trials were taken as hits. The higher the Sensitivity the better was the target seen. There are inter-individual differences in the Sensitivity (Figure 7.4, page 94). Some participants (e.g. number 1, 14, or 27) show an increasing course with increasing SOA and therefore see the target most clearly in long SOAs. Those participants can be assumed to be Type A observers. Others (e.g. number 2, 18, or 29) have a decreasing course and therefore see the target most clearly in short SOAs. Those participants can be assumed to be Type B observers. The grand average has a J-like course with significant variability over SOAs (main effect SOA: $F(5,145)=8.25, p=.005$ ).

Response Bias. The Response Bias $C_{R}$ reflects the tendency to answer that the target was

or not clearly seen. Positive values reflect the bias to answer that they have not seen the target (conservative response) and negative values reflect the bias to answer that they have seen the target (liberal response). Most participants show a positive Bias (Figure 7.4, page 94), which is either increasing (e.g. 2, 3, or 29), decreasing (e.g. 10, 22, or 27), or equal (e.g. 7, 19, or 30) over SOAs. Therefore, masking is effective and reduces the visibility of the target. For those with a decreasing course the target is best visible in long SOAs and therefore it can be assumed that they might be Type A observers, and for those with an increasing course the target is best visible in short SOAs and therefore it can be assumed that they might be Type B observers. Number 20 has nearly no Bias and Number 21 has a slightly negative Bias equally distributed over SOAs and therefore tend to answer that the target was seen. The grand average shows a positive response bias decreasing over SOAs (main effect SOA: $F(5,145)=8.25, p<.005$ ). 
(a)

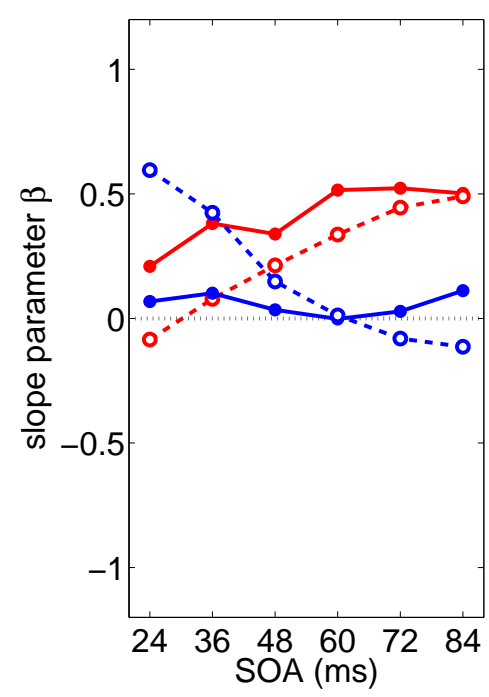

(b)

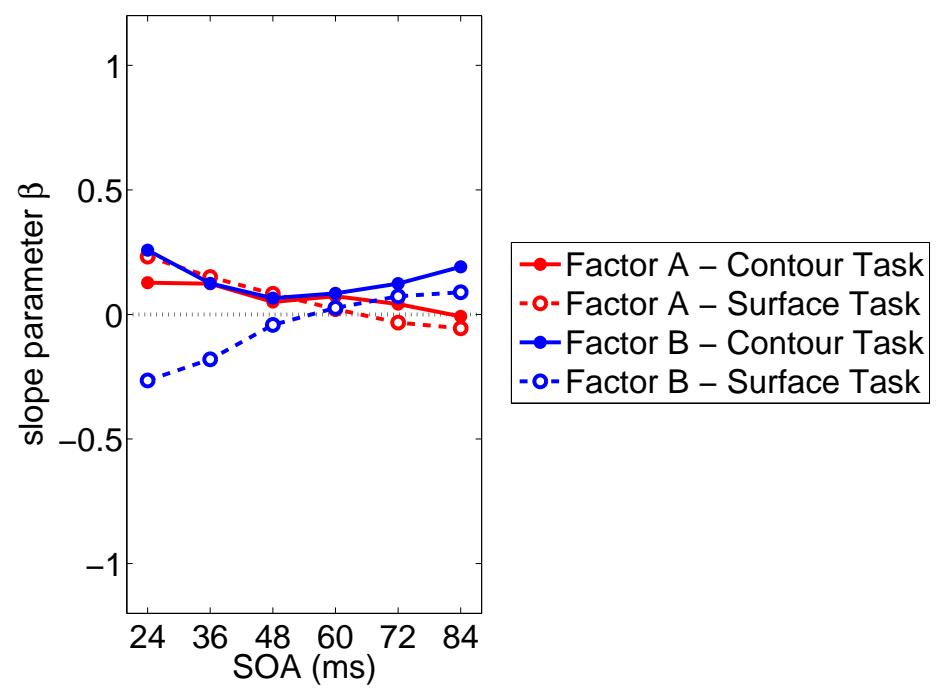

Figure 7.5: Experiment 5.2. Slope parameter $\beta$ over SOAs, with (a) Discrimination Sensitivity $d^{\prime}$ and (b) Mask Bias $C_{R}$ values correlated with Factor A scores and Factor B scores.

\section{Correlation of Discrimination Sensitivity and Mask Bias With Individual Factor Scores}

The slope parameter $\beta$ of the correlations of the factor scores with $d^{\prime}$ and $C_{R}$ values are shown in Figure 7.5 (page 96). The impact of Factor A on Sensitivity is low in short SOAs and increasing with increasing SOA in both tasks and with higher impact in the contour task. While the increase of impact over SOA becomes significant (two-way interaction Factor A x SOA:F $(5,135)=6.23$, $p=.006$ ), there is no interaction of Factor $\mathrm{A}$ with the task (two-way interaction Factor $\mathrm{A} \mathrm{x}$ Task: $F(1,27)=0.71, p=.408$ ) and no further interaction with the task and SOA (three-way interaction of Factor A x SOA x Task: $F(5,135)=1.39, p=.255)$. In the contour task the Factor B has nearly no impact in all SOAs. By contrast, in the surface task Factor B has high impact in short SOAs decreasing with increasing SOAs, and low impact in intermediate and long SOAs. As there is no interaction between Factor B and task $(F(1,27)=0.30, p=.586)$, the impact depends on different SOAs for both tasks (three-way interaction of Factor B x SOA x task: $F(5,135)=8.06, p<.001)$.

The impact of Factor A on the Response Bias in both tasks is small in short SOAs and decreasing with increasing SOA to no impact at all. Like for the Sensitivity, while the increase of impact over SOA becomes significant (two-way interaction Factor A x SOA: $F(5,135)=4.78$, $p=.014$ ), there is no interaction of Factor A with the task (two-way interaction Factor A $\mathrm{x}$ Task: $F(1,27)<0.00, p=.990)$ and no further interaction with the task and the SOA (three-way interaction of Factor A x SOA x Task: $F(5,135)=1.25, p=.292)$. In the contour task Factor B 
has a positive impact in a flat U-course with stronger impact in short and long SOAs and less in intermediate SOAs. In contrast, during the surface task, Factor B has a negative impact in short SOAs increasing with increasing SOA and a small positive impact in long SOAs. Like for the Sensitivity, as there is no interaction between Factor B and task $(F(1,27)=2.51, p=.125)$, the impact depends on different SOAs for both tasks (three-way interaction of Factor B x SOA $\mathrm{x}$ task: $F(5,135)=9.62, p<.001)$.

\subsection{Discussion}

\subsubsection{Experiment 5.1}

This experiment aimed to obtain inter-individual differences in the Discrimination Sensitivity using them for a factor analysis, whose factors represent potential processes involved in metacontrast masking. Like in Study 1 to 4 inter-individual differences in metacontrast masking occurred, which were further used for a factor analysis like in Study 1 to 3, which revealed two underlying latent variables, explaining $88 \%$ of total variability. The Factor A variable is increasing with increasing SOA, explaining mainly data of intermediate and long SOAs, and the Factor B variable is decreasing with increasing SOA, explaining mainly data of short SOAs. As described in Study 1, these factors may be regarded as reflecting two independent (perceptual) latent processes, that jointly determine the shape of the masking function. More specifically, it is assumed that Factor A represents a segregation process, resulting in a percept of two succeeding stimuli, and that Factor B represents an integration process, resulting in a percept of a simultaneous, conjoint presentation of both stimuli. Both Factors have an impact on the manifestation of the Mask Bias $C_{M}$ : Factor A mainly in short SOAs and Factor B's impact is more evenly distributed over all SOAs, though it is relatively small. In contrast to the Studies 1 to 3, two factors were not sufficient to explain the data. However, a third factor describes less than one SOA, so it may correspond a confounder. It may be worth a try to run a factor analysis with three factors and regarding the third factor as an aggregate of confounding variables that will be omitted for further analysis. In this way the other two variables may be more pure in their data explanation even if less variance may be explained.

\subsubsection{Experiment 5.2}

In this experiment metacontrast stimuli were presented with varying SOA but with two different tasks. In one task participants had to report if they saw a rotational movement in the sequence of target and mask, and in the other task they had to judge how clear they saw the target stimulus. When comparing all three experiments $(5.1,5.2 .1$, and 5.2.2) some coherences are apparent: 


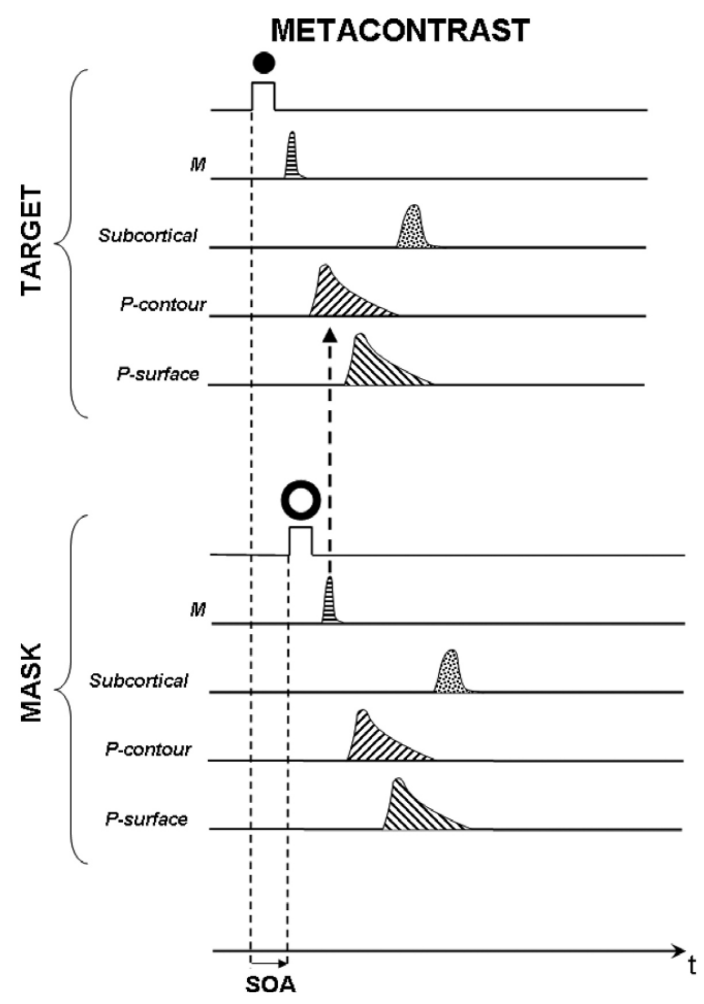

Figure 7.6: Schematic diagram of the optimal metacontrast suppression effect of a mask on the contour and brightness visibilities of a prior target stimulus. Dashed vertical arrow indicates inhibition of the target's sustained activity by the mask's transient activity. Illustration and description from Breitmeyer et al. (2006).

Participants who report to see the target more clearly in long SOAs also have higher Sensitivity to detect rotations in long SOAs. Participants who are Type B in Experiment 5.1 report to see the target more clearly in short SOAs, while participants who are Type A in Experiment 5.1 report to see the target more clearly in long SOAs and have higher Sensitivity to detect rotations in long SOAs. Rotations were as expected better perceived in long SOAs than in short SOAs. Surprisingly, all participants were able to detect rotations. This is unexpected as in the last studies only few participants who showed a Type B masking function reported to perceive rotations. Therefore, when introducing the task to detect rotations all seem to find perceptual cues indicating to a apparent rotation in some trials. However, it is unknown what participants actually perceived during the task. It might be that some used other criterion contents to discriminate between congruent and incongruent tasks.

According to the theory of Breitmeyer et al. (2006) contours and surfaces were separately processed via the parvocellular path, namely contours earlier than surfaces (see Figure 7.6, page 98). The illustrated model shows that the transient path of the mask inhibits via interchannel inhibition only the sustained processing of the contours but not those of the surface. 
Therefore, stimulus surface information is still processed while the contour information is masked. When assuming that this interaction happens in a relatively short SOA, then it might be that in a longer SOA surface is inhibited instead of the contours. In intermediate SOAs both processes might be inhibited resulting in a maximal masking, observed in a U-shaped masking function. When in short SOAs the sustained contour processing is inhibited the sustained surface processing still remains. Similarly, when in long SOAs the sustained surface processing is inhibited, the contours could already be processed.

How can inter-individual differences be explained in the context of this theory? As the influence of Process A increases with increasing SOA, this process might support the processing of contours. This support might be a temporal segregation of two subsequently appearing stimuli enabling the detection of motion perceptions. Therefore, individuals with a prominent Process A might more often perceive apparent rotations, which in turn can be used for task fulfillment, and therefore have higher Sensitivity in long SOAs. As this finer segregation might enable a better perception of the target, Sensitivity is higher in long SOAs when it has to be judged how clear the target is perceived. Individuals with a weak Process A cannot profit from this supported contour processing and have lower Sensitivity in long SOAs. The influence of Process A on the Response Bias is weak but with a prominent Process A the bias tends to report no rotation detections in short SOAs. As there is no influence of Process B in the contour task, this process can be assumed to have no influence on contour processing. However, in the surface task it influences the Sensitivity in in short SOAs and therefore might support the processing of surface information. This support might enable the perception of negative afterimages. Therefore, individuals with a prominent Process B might perceive these afterimages so that they see the target more clearly in short SOAs. Individuals with a weak Process B cannot profit from this supported surface processing and have lower Sensitivities in short SOAs. Processes B has no influence on the Response Bias in the contour task but strong influence in short SOAs. In the surface task it enhances the bias to respond that the target was clearly seen. The results strongly indicate specialization of Process A to long SOAs and of Process B to short SOAs. It seems that Process A and B are only active when contours and surfaces are processed, respectively. This may indicate that those processes do not independently interact with the sustained and transient channels but become active in later processing levels, influencing the conscious stimulus perception. 


\subsection{Conclusion}

This study aimed to investigate if inter-individual differences with different instructions of the task. The tasks were to detect apparent rotations and to judge the clarity of the target. Apparent rotations in the sequence of target and mask can be better perceived in long SOAs than in short SOAs. The clarity of the perception of the target mainly corresponds to the masking function in a target discrimination task. Participants who showed a Type B masking functions in a target discrimination task also report to perceive the target more clearly in short SOAs, while those who showed a Type A masking function in a target discrimination task also report to perceive the target more clearly in long SOAs. The impact of Process A increases with increasing SOAs for both tasks and therefore might support the processing of contours by enabling motion perception. Process B is ineffective in the contour task and therefore seems to be not involved in contour processing. However, as it is most effective in short SOAs of the surface task it might support the processes of surface processing by the perception of afterimages. 


\section{Overall Summary and Discussion}

\subsection{Metacontrast Masking}

In metacontrast masking the visibility of the first stimulus (the target) is reduced due to the appearance of the second stimulus (the mask). Both stimuli are not overlapping, neither temporally nor spatially. It is assumed that different stimulus features are processed via two different visual paths (Breitmeyer \& Öğmen, 2006): A fast, transient path processes the presence and location of a stimulus, while a slower, sustained path processes features like contours and surfaces of the stimulus. Masking may occur as the transient path of the mask inhibits the sustained path of the target and therefore impairs its processing. However, it is not yet fully understood which effects this inhibition has on further target processing. For the masking effect timing is crucial. The time span between target onset and mask onset (SOA) deliberately determines the degree of visibility. Measuring the effect over a series of different SOAs gives a time course of the processing of the target, where processing characteristics can be inferred. A masking function, where visibility is increasing with increasing SOA, is called "Type A masking function". A masking function, where visibility is high in short and long SOAs and dropped to a minimum in between, is called "Type B masking function". Which function is obtained depends on experimental parameters. By manipulating them a shift from one type to the other may be effected.

In addition, different phenomenological percepts were perceived by individual participants, when performing a target shape discrimination task. These perceptions serve as criterion contents that were used as indices for the shape of the target. The different criterion contents go along with Type A and Type B masking functions, respectively. Participants who show a Type A masking function mostly report to see and use apparent movements in the sequence of both stimuli, mainly in long SOAs, while those who show a Type B masking function mostly report to see and use negative afterimages in the shape of the target inside the mask, mainly in short SOAs. Examining these inter-individual differences may contribute to the question how different target features are processed despite the inhibition of the mask. 


\subsection{Studies}

In five studies it was examined which influence different experimental parameters have on metacontrast masking functions. Four stimulus parameters were varied: the SOA, the presentation durations of target and mask, the presentation eccentricity and the presentation sizes of target and mask. Two attentional manipulations were made: a spatial uncertainty of the presentation position, where attention had to be distributed over a larger or smaller area, and a top-down selection of attention on either the metacontrast stimuli or the color changing fixation point. And four different tasks were varied: a shape discrimination task, a color change detection task, an apparent rotation detection task, and a perception awareness judgment. In all studies the same stimulus material was used, and the SOA was varied to examine the temporal course of the masking effect. How the masking effect varies over different SOAs crucially characterizes the interaction of both stimuli. By manipulating further parameters, the course of the masking function changes, which in turn gives indications of the characteristics of the mechanisms involving metacontrast masking. The variations described above were chosen as it was assumed that their influence enables a profound descriptions of these characteristics.

In one study datasets were categorized to a masking function of Type A or Type B according to their behavioral performance. These two groups could then be compared in their physiological response to the stimuli in two attention manipulations. In the other studies, datasets were linearly assigned on factors, obtained in a factor analysis, which correspond to mechanism leading either to an Type A or Type B masking function.

\subsection{Results}

\subsubsection{Effects of Parameter Manipulation on Behavioral Performance}

Masking functions are determined by an interaction of the target-to-mask (T/M) energy ratio and the target duration. The longer the mask duration becomes in relation to the target duration (the smaller the T/M ratio), the more the minimum of the masking function shifts towards shorter SOAs, and the more Type-A-like (monotonically increasing) the function becomes. This replicates findings described by Weisstein (1972) and Breitmeyer (1978). By increasing the target duration for all mask durations the Discrimination Sensitivity increases for all SOAs but most prominent in short and intermediate SOAs, shifting the minimum of the curve, where maximal masking occurs, towards longer SOAs. Within a fixed target duration maximal masking occurs at shorter SOAs the longer the mask duration is.

Masking functions depend on stimulus size as well as eccentricity. In the center and in the periphery masking is stronger in small stimuli than in large stimuli. Furthermore, the SOA of 
the strongest masking occurred at the shortest SOA in both stimulus sizes. Stimulus size and eccentricity have no interacting effect by themselves, but their interaction varies in dependence of the SOA. Thus, the study by Bridgeman and Leff (1979) could be replicated partially.

Masking functions are identical for predictable and unpredictable stimulus presentation locations. Thus, distributing the spatial attention over a large area in contrast to focusing attention on a smaller area does not affect the masking effect. However, considering the minima of the masking functions it shifts toward longer SOAs when attention is more distributed and stimuli are presented in the fovea than when attention is more focused, and it shifts toward shorter SOAs when attention is more distributed and stimuli are presented in the periphery than when attention is more focused.

In all experiments with the target shape discrimination task in which only the SOA was varied, inter-individual differences were found with individual masking functions of either Type A, Type B or intermediate courses. These findings replicate those of Albrecht et al. (2010) and Albrecht and Mattler (2012a, 2012b). Color change detection does not differ between SOAs indicating that not attended metacontrast masking stimuli are not interfering with this task. Apparent motion is better detected in long SOAs than in short SOAs. Participants who report to see the target more clearly in long SOAs have also higher Sensitivity to detect rotations in long SOAs. Participants who are Type B in the shape discrimination task report to see the target more clearly in short SOAs, while participants who are Type A in the shape discrimination task report to see the target more clearly in long SOAs and have higher Sensitivity to detect rotations in long SOAs.

\subsubsection{Physiological Comparison of Types}

Type A and Type B observers do not differ in their bottom-up processing of metacontrast stimuli, that is, when stimuli were presented but attention was not directed to them. But they do differ in their top-down processing, that is, when attention was on stimuli and the target had to be discriminated by its shape. Therefore, in Type A and B observer target and mask stimuli were differently processed, which might explain their different perceptions. Group differences were found between 200 and $400 \mathrm{~ms}$ after target onset at electrode sites PO7/PO8 and O1/O2. Type $\mathrm{B}$ observers show a more negative ERP when attending metacontrast stimuli than when not, which is more pronounced in the short than in the long SOA. This might refer to an enhanced visibility of the target induced by a negative afterimage. In contrast, Type A observers show more positive ERPs especially in the long SOA, which might be comparable related to a perception, for example apparent motion. 


\subsection{Processes Involved in Metacontrast Masking and Their Characteristics}

\subsubsection{Revealing Two Processes}

The factor analysis is a data reduction procedure, which estimates the number variables that are sufficient to describe the individual variability in masking functions. Its factors represent potential processes involved in metacontrast masking. Data patterns can be described well by two underlying latent variables explaining between 88 and $93 \%$ of total variability. The first latent variable reflects an increasing masking function, the second one reflects a decreasing masking function. The study by Albrecht and Mattler (2016), in which a similar factor structure with two factors explaining $88 \%$ of total variability corroborates this finding. The two factors load differently high on the single SOAs. Loadings of Factor 1 are increasing with increasing SOA explaining predominantly data of long SOAs. As it describes the process leading to a Type A masking function it is named "Factor A". Loadings of Factor 2 are decreasing with increasing SOA explaining predominantly data of short SOAs. As it describes the process leading to a Type B masking function it is named "Factor B". The individual factor scores represent how well the dataset is described by the two factors. When correlated to individual Discrimination Sensitivity and Mask Bias values, it can be inferred how the factor scores influence the masking and bias functions. A high score on Factor A correlates with high Sensitivity in long SOAs and in a high positive Mask Bias in short SOAs. A high score on Factor B correlates with high Sensitivity and a negative Mask Bias in short SOAs. Depending on high or low Factor A and B scores the individual masking function is formed.

The criterion content describes the stimulus dimension along which the perceptual judgment about the target is made (Kahneman, 1968). Type A and Type B observers describe to perceive and use different criterion contents when fulfilling the target shape discrimination task (Albrecht $\&$ Mattler, 2012b). Type A observer report to see and use percepts of apparent motion, a rotating movement in incongruent trials and an expanding movement in congruent trials, while Type B observers report to see and use percepts of negative afterimages as a conjoint percept of target and mask. As different criterion contents lead to different masking functions (Breitmeyer \& Öğmen, 2006) they are useful information, which can be used to infer the underlying processes of Factor A and B.

The process represented by Factor A is strongly associated with a Type A masking function, which in turn goes along with a percept of motion. According to the Integration-SegregationApproach by Neumann (1978) and Reeves (1982) the perception of two stimuli being segregated, enabling apparent moving images are associated to the segregation process. Thus, the Factor A process may correspond to the segregation process (Process A). Furthermore, the pro- 
cess represented by Factor B is strongly associated with a Type B masking function, which in turn goes along with a percept of negative afterimages inside of the mask stimulus. The percept of simultaneous, conjoint image of two physically succeeding stimuli are associated to the integration process of the same model and thus, the Factor B process may correspond to the integration process (Process B).

\subsubsection{Characteristics of Processes}

Correlating the individual factor scores with the Discrimination Sensitivity of the different experimental manipulations expresses the involvement of the two processes in the formation of the masking functions. This knowledge enables to characterize the involved mechanisms. Based on the Dual-Channel-Model by Breitmeyer et al. (2006), stimulus contours are processed faster via the sustained path than stimulus surfaces. In the model illustrated in Figure 7.6 (page 98) the transient path inhibits only the sustained processing of the contours. When assuming that this interaction happens in a relatively short SOA then it might be that in a longer SOA surface is inhibited instead of the contours. Therefore, not all stimulus information of the target are inhibited and may be further processed. The integration and the segregation process, might be fed by the non-inhibited sustained information and might lead to, or support a conscious percept. More specifically, the integration process supports the surface information when the contour information are inhibited (in short SOAs). And the segregation process supports the contour information when the surface information are inhibited (in long SOAs). Both processes are not equally activated in every experimental condition, though. The characteristics of both processes are summarized as described below.

In general, segregation process is most influencing in long SOAs with decreasing impact the shorter the SOA becomes. While in long SOAs its impact is equally high for different target and mask durations, its influence increases even in intermediate and short SOAs with increasing mask duration. When stimuli are presented in the periphery rather than in the foveal region the segregation process becomes less influencing, but has more influence in long SOAs. The segregation process is effective in all stimulus sizes, but is more effective in larger than in smaller stimuli, especially in long SOAs. In foveal positions its influence is lower when attention is distributed than when attention is more focused. In the periphery attentional distribution is not dependent. In long SOAs the segregation process influences the processing of stimulus contours and surfaces, with decreasing SOAs this influence decreases but lesser in contours than in surfaces.

In general, the integration process is most influencing in short SOAs with decreasing impact the longer the SOA becomes. In short mask durations it is most influencing in intermediate SOAs and with increasing mask durations this influence shifts to short SOAs. With increasing 
target durations its influence increases in longer SOAs. The integration process has low influence in the periphery being most effective in the foveal visual field. It is more influencing with increasing stimulus size, especially in short SOAs. Distributing or focusing the attention does not effect the influence of the integration process. In short SOAs it influences the processing of stimulus surface. With increasing SOAs this influence decreases. It does not influence the processing of the contours.

\section{Training and Context Effects}

In the Studies 1 to 3 it was additionally compared if individual masking functions change over sessions. Here, stimuli were always presented foveally (and therefore were predictable in their position), and with intermediate size (compared to other size variations). In all studies there was an general increase of the performance level after several sessions performing the discrimination task. This general increase is also described by Schwiedrzik et al. (2009). In addition, after performing six sessions where target and mask durations were varied, performance level increased especially in short SOAs, uplifting the decreasing branch of the masking function, turning it into a Type B function. In contrast, variations of eccentricity, stimulus size, and spatial attention seem to have no effect as individual masking functions did not change. Therefore, variations of the target-to-mask ratio seem to have special influence on the improvement of Sensitivity in short SOAs. An increase of awareness of the target might have lead to this increase of Sensitivity, and the target-to-mask ratio variations might have trained this awareness (Schwiedrzik et al., 2009 and Ventura, 1980 showed that awareness can be trained). The perceptual integration of target and mask to a conjoint percept associated with negative afterimages similarly enhances the awareness of the target. Therefore, it may be that the Process B, the integration process, was especially trained in all participants leading to an increase in Sensitivity in short SOAs. This also is consistent to the finding that the Type B group showed more negative ERPs in short SOAs indicating an enhanced visibility of the target (Railo \& Koivisto, 2009).

\subsection{Proposals for Further Research}

\subsubsection{Proposals for Additional Analyses}

In the following, additional analysis are suggested, which may give further hints for understanding the mechanisms involved in metacontrast masking. In Study 1, next to the SOA it might be insightful to sort data after the stimulus-termination-asynchrony to illustrate the effect of the mask duration in more detail, and to sort data after the inter-stimulus-interval to illustrate the effect of independent of target duration. In Study 2 and 3 an eyetracker was used to control for 
eye positions at the beginning of each trial. Nevertheless, those data are recorded during the whole trial and it may be interesting to analyze them and find characteristic patterns for Type A and B observer. In Study 4 trials without mask were presented. Those trials could be used to calculate a difference curve, where the ERPs of target-only trials were subtracted from trials where target and mask stimuli were presented. As a result, only the ERPs referring to mask processing remain and may give more detailed information of possible processing differences between Type A and B observers. Furthermore, in contrast to Study 1 to 3 and 5, in Study 4 data were compared between two distinct groups, namely Type A and B observer. It may be insightful to conduct a factor analysis as well and correlate the individual Factor A and B scores with the $\mu \mathrm{V}$ values of the ERPs. In Study 5 participants rated their perception of the target using the Perception-Awareness-Scale. After the session the individual categories participants assigned to the four scale points were wrote down. These descriptions of the perceptions could be independently rated and categorized to groups of perceptions. It would be interesting if a correlation of perception descriptions and masking functions could be found.

\subsubsection{Proposals for new Experiments}

In addition, in the following some further experiments are suggested, which might contribute to the understanding of the development of inter-individual differences. Participants report that when they have once established their criterion content, perception of apparent motion or negative afterimages, they focus their attention on these percepts. As afterimages appear inside the inner contours of the mask, Type B observers focus their attention to this area. In contrast, to detect motions the attention has to be on the outer contours of both stimuli. Based on this, it can be assumed that Type A observer have a more distributed or more "global" attention, while Type B observer have more focused or more "local" attention. An initial training with a short SOA leads to an increase of performance in this particular SOA in all participants, while an initial training with a long SOA leads to an increase of performance in this particular SOA only in some participants (Albrecht \& Mattler, 2014). It may be concluded that a training on a SOA per se does not induce the percepts used as criterion contents. However, to guide their attention so that they may be better able to establish specific criterion contents, may enable to control masking functions. This could be realized by two possible experimental settings. First, instead of a fixation cross, a fixation ring in two different diameters could be used to which attention has to be drawn. This would be either as large as the outer contours of the mask so that attention is automatically distributed. This may effect a Type A masking function. Or it would be smaller fitting inside the inner contours of the mask so that attention is automatically focused. This may effect a Type B masking function. Second, Navon stimuli could be used for training (Navon, 1977). One group of participants would be trained to identify the local figures and the other 
group would be trained to identify the global figures. Similar to the first alternative, it would be expected that participants trained for local figures more likely develop a Type B masking function, and those trained for global figures would develop more likely a Type A masking function.

Inter-individual differences in Sensitivity and criterion content were observed when participants performed the target-discrimination task. To further examine the physiological differences between participants, an experiment might be insightful, where ERPs were compared when participants had their attention on metacontrast stimuli and either perform the discrimination task or not. Results may answer the questions if individual phenomenological perceptions occur even without task, if ERPs differ between participants without task and how ERPs differ between participants with task (as replication of Study 4). It may even be compared how ERPs change from trial to trial when the criterion content evolves (although data quality might be insufficient due to too few trials).

To specifically assign ERPs to specific perceptions, an EEG experiment could be conducted, where participants have to rate the clarity of the target using the perception-awareness-scale (as in Study 5). ERPs of participants who used the same scale classification (with the same perceptions) could be grouped together, and different groups could be compared.

To test if results obtained with metacontrast masking and the stimuli used in the present studies, are generally valid, other stimuli and masking methods should be used and results compared.

\subsection{Concluding Remarks}

The present dissertation systematically investigated the occurrence of different masking functions in metacontrast masking. Experiments were based on fundamental studies, which findings could be mostly verified and even extended by further parameters. Furthermore, inter-individual differences were used to get insights in the mechanisms involved in metacontrast masking. Two processes could be revealed, which may be involved in the conscious perception of the target stimulus in metacontrast masking. 


\section{References}

Akyürek, E. G., Schubö, A., \& Hommel, B. (2010). Fast temporal event integration in the visual domain demonstrated by event-related potentials. Psychophysiology, 47(3), 512522. doi: 10.1111/j.1469-8986.2010.00962.x

Albrecht, T., Klapötke, S., \& Mattler, U. (2010). Individual differences in metacontrast masking are enhanced by perceptual learning. Consciousness and Cognition, 19(2), 656-666. doi: 10.1016/j.concog.2009.12.002

Albrecht, T., \& Mattler, U. (2012a). Individual differences in metacontrast masking regarding sensitivity and response bias. Consciousness and Cognition, 21(3), 1222-1231. doi: 10.1016/j.concog.2012.04.006

Albrecht, T., \& Mattler, U. (2012b). Individual differences in subjective experience and objective performance in metacontrast masking. Journal of Vision, 12(5), 1-24. doi: $10.1167 / 12.5 .5$

Albrecht, T., \& Mattler, U. (2014). Qualitative individuelle Unterschiede bei Metakontrastmaskierung als Zugang zur Erforschung der Mechanismen der bewussten Wahrnehmung: (Verlängerugnsantrag auf Sachmittel an die DFG). Göttingen: Georg-August-Universität Göttingen, Abteilung Experimentelle Psychologie.

Albrecht, T., \& Mattler, U. (2016). Individually different weighting of multiple processes underlies effects of metacontrast masking. Consciousness and Cognition, 42, 162-180. doi: 10.1016/j.concog.2016.03.006

Andreassi, J., Simone, J. d., \& Mellers, B. (1976). Amplitude changes in the visual evoked cortical potential with backward masking. Electroencephalography and Clinical Neurophysiology, 41(4), 387-398. doi: 10.1016/0013-4694(76)90101-2

Ansorge, U., Becker, S. I., \& Breitmeyer, B. G. (2008). Revisiting the metacontrast dissociation: Comparing sensitivity across different measures and tasks. The Quarterly Journal of Experimental Psychology, 62(2), 286-309. doi: 10.1080/17470210801908492

Ansorge, U., Breitmeyer, B. G., \& Becker, S. I. (2007). Comparing sensitivity across different processing measures under metacontrast masking conditions. Vision Research, 47(27), 3335-3349. doi: 10.1016/j.visres.2007.09.009

Bachmann, T., \& Francis, G. (2013). Visual Masking: Studying Perception, Attention, and Consciousness. Burlington: Elsevier Science. 


\section{References}

Bashinski, H. S., \& Bacharach, V. R. (1980). Enhancement of perceptual sensitivity as the result of selectively attending to spatial locations. Perception $\mathcal{E}$ Psychophysics, 28(3), 241-248. doi: 10.3758/BF03204380

Beck, J., \& Ambler, B. (1973). The effects of concentrated and distributed attention on peripheral acuity. Perception \& Psychophysics, 14(2), 225-230. doi: 10.3758/BF03212381

Breitmeyer, B. G. (1978). Metacontrast masking as a function of mask energy. Bulletin of the Psychonomic Society, 12(1), 50-52. doi: 10.3758/BF03329621

Breitmeyer, B. G., \& Ganz, L. (1976). Implications of sustained and transient channels for theories of visual pattern masking, saccadic suppression, and information processing. Psychological Review, 83(1), 1-36. doi: 10.1037/0033-295X.83.1.1

Breitmeyer, B. G., Kafalıgönül, H., Öğmen, H., Mardon, L., Todd, S., \& Ziegler, R. (2006). Meta- and paracontrast reveal differences between contour- and brightness-processing mechanisms. Vision Research, 46(17), 2645-2658. doi: 10.1016/j.visres.2005.10.020

Breitmeyer, B. G., \& Öğmen, H. (2000). Recent models and findings in visual backward masking: A comparison, review, and update. Perception E Psychophysics, 62(8), 15721595. doi: 10.3758/BF03212157

Breitmeyer, B. G., \& Öğmen, H. (2006). Visual masking (2nd ed., Vol. 41). Oxford: Oxford University Press.

Breitmeyer, B. G., \& Tapia, E. (2011). Roles of contour and surface processing in microgenesis of object perception and visual consciousness. Advances in cognitive psychology/University of Finance and Management in Warsaw, 7, 68-81. doi: 10.2478/v10053-008-0088-y

Bridgeman, B. (1988). Visual evoked potentials: Concomitants of metacontrast in late components. Perception $\mathcal{E}$ Psychophysics, 43(4), 401-403. doi: 10.3758/BF03208812

Bridgeman, B., \& Leff, S. (1979). Interaction of stimulus size and retinal eccentricity in metacontrast masking. Journal of Experimental Psychology: Human Perception and Performance, 5(1), 101-109. doi: 10.1037/0096-1523.5.1.101

Bruchmann, M., Hintze, P., \& Mota, S. (2011). The effects of spatial and temporal cueing on metacontrast masking. Advances in Cognitive Psychology, 7(1), 132-141. doi: 10.2478/ v10053-008-0093-1

Coles, M. G. H., \& Rugg, M. D. (1995). Event-related brain potentials: An introduction. In M. D. Rugg \& M. G. H. Coles (Eds.), Electrophysiology of mind (Vol. no. 25). New York: Oxford University Press.

Cox, S. I., Dember, W. N., \& Sherrick, M. F. (1969). Effect on backward masking of spatial separation between target and mask contours and of target size. Psychonomic Science, 17(4), 205-206. doi: 10.3758/BF03329171 
Delorme, A., \& Makeig, S. (2004). EEGLAB: an open source toolbox for analysis of singletrial EEG dynamics including independent component analysis. Journal of neuroscience methods, 134(1), 9-21. doi: 10.1016/j.jneumeth.2003.10.009

Di Lollo, V., Mühlenen, A. v., Enns, J. T., \& Bridgeman, B. (2004). Decoupling stimulus duration from brightness in metacontrast masking: data and models. Journal of experimental psychology. Human perception and performance, 30(4), 733-745. doi: 10.1037/0096-1523.30.4.733

Didner, R., \& Sperling, G. (1980). Perceptual delay: A consequence of metacontrast and apparent motion. Journal of Experimental Psychology: Human Perception and Performance, 6(2), 235-243. doi: 10.1037/0096-1523.6.2.235

Dixon, P., \& Dilollo, V. (1994). Beyond Visible Persistence: An Alternative Account of Temporal Integration and Segregation in Visual Processing. Cognitive Psychology, 26(1), 33-63. doi: 10.1006/cogp.1994.1002

Duangudom, V., Francis, G., \& Herzog, M. H. (2007). What is the strength of a mask in visual metacontrast masking? Journal of Vision, 7(1), 7. doi: 10.1167/7.1.7

Enns, J. T., \& Di Lollo, V. (1997). Object Substitution: A New Form of Masking in Unattended Visual Locations. Psychological science, 8(2), 135-139. doi: 10.1111/j.1467-9280.1997 .tb00696.x

Eriksen, C. W., Becker, B. B., \& Hoffman, J. E. (1970). Safari to masking land: A hunt for the elusive U. Perception E Psychophysics, 8(4), 245-250. doi: 10.3758/BF03210215

Fehrer, E., \& Smith, E. (1962). Effect of luminance ratio on masking. Perceptual and Motor Skills(14).

Francis, G. (2003). Developing a new quantitative account of backward masking. Cognitive Psychology, 46(2), 198-226. doi: 10.1016/S0010-0285(02)00521-2

Francis, G., \& Cho, Y. S. (2008). Effects of temporal integration on the shape of visual backward masking functions. Journal of Experimental Psychology: Human Perception and Performance, 34(5), 1116-1128. doi: 10.1037/0096-1523.34.5.1116

Geerligs, L., \& Akyürek, E. G. (2012). Temporal integration depends on increased prestimulus beta band power. Psychophysiology, 49(11), 1464-1467. doi: 10.1111/j.1469-8986.2012 .01453.x

Growney, R. (1976). The function of contour in metacontrast. Vision Research, 16(3), 253-261. doi: 10.1016/0042-6989(76)90108-5

Handy, T. C. (2004). Event-related potentials: A methods handbook. Camridge, Mass.: MIT Press. 


\section{References}

Johnson, C. A., Keltner, J. L., \& Balestrery, F. (1978). Effects of target size and eccentricity on visual detection and resolution. Vision Research, 18(9), 1217-1222. doi: 10.1016/ 0042-6989(78)90106-2

Kahneman, D. (1967). An onset-onset law for one case of apparent motion and metacontrast. Perception $\mathcal{E}$ Psychophysics, 2(12), 577-584. doi: 10.3758/BF03210272

Kahneman, D. (1968). Method, findings, and theory in studies of visual masking. Psychological Bulletin, 70(6, Pt.1), 404-425. doi: 10.1037/h0026731

Kaiser, P. (2016). Temporal Summation (Bloch's Law). http://www.yorku.ca/eye/blochlaw.htm.

Klopp, E. (2010). Explorative Faktorenanalyse. http://hdl.handle.net/20.500.11780/3369.

Kolers, P. A. (1962). Intensity and contour effects in visual masking. Vision Research, 2(9-10), 277-IN4. doi: 10.1016/0042-6989(62)90037-8

Lee, B. B., Martin, P. R., \& Grunert, U. (2010). Retinal connectivity and primate vision. Progress in retinal and eye research, 29(6), 622-639. doi: 10.1016/j.preteyeres.2010.08 .004

Lingnau, A., \& Vorberg, D. (2005). The time course of response inhibition in masked priming. Perception $\mathcal{E}$ Psychophysics, 67(3), 545-557. doi: 10.3758/BF03193330

Loftus, G. R., \& Masson, M. E. (1994). Using confidence intervals in within-subject designs. Psychonomic bulletin E review, 1(4), 476-490. doi: 10.3758/BF03210951

Lopez-Calderon, J., \& Luck, S. J. (2014). ERPLAB: an open-source toolbox for the analysis of event-related potentials. Frontiers in human neuroscience, 8, 213. doi: 10.3389/ fnhum.2014.00213

Macknik, S. L., \& Livingstone, M. S. (1998). Neuronal correlates of visibility and invisibility in the primate visual system. Nature Neuroscience, 1(2), 144-149. doi: 10.1038/393

Macmillan, N. A., \& Creelman, C. D. (2005). Detection theory: A user's guide (2nd ed ed.). Mahwah, N.J: Lawrence Erlbaum Associates.

MATLAB R2013a (version 8.1.0.604) [Computer software]. (2013). Natick, Massachusetts: The MathWorks, Inc.

Matteson, H. H. (1969). Effects of Surround Size and Luminance on Metacontrast*. Journal of the Optical Society of America, 59(11), 1461. doi: 10.1364/JOSA.59.001461

Montagna, B., Pestilli, F., \& Carrasco, M. (2009). Attention trades off spatial acuity. Vision Research, 49(7), 735-745. doi: 10.1016/j.visres.2009.02.001

Navon, D. (1977). Forest before trees: The precedence of global features in visual perception. Cognitive Psychology, 9(3), 353-383. doi: 10.1016/0010-0285(77)90012-3

Neumann, O. (1978). Visuelle Aufmerksamkeit und der Mechanismus des Metakontrasts.

Neumann, O., \& Scharlau, I. (2006). Visual attention and the mechanism of metacontrast. Psychological research, 71(6), 626-633. doi: 10.1007/s00426-006-0061-7 
Paradiso, M. A., \& Nakayama, K. (1991). Brightness perception and filling-in. Vision Research, 31(7-8), 1221-1236. doi: 10.1016/0042-6989(91)90047-9

Petry, S. (1978). Perceptual changes during metacontrast. Vision Research, 18(10), 1337-1341. doi: 10.1016/0042-6989(78)90224-9

Presentation (version 16.1) [Computer software]. (2012). Berkeley, CA: Neurobehavioral Systems, Inc.

R Core Team (version 3.0.1) [Computer software]. (2013). Vienna, Austria: R Foundation for Statistical Computing.

Railo, H., \& Koivisto, M. (2009). The electrophysiological correlates of stimulus visibility and metacontrast masking. Consciousness and Cognition, 18(3), 794-803. doi: 10.1016/ j.concog.2009.01.006

Ramachandran, V. S., \& Cobb, S. (1995). Visual attention modulates metacontrast masking. Nature, 373(6509), 66-68. doi: 10.1038/373066a0

Ramsøy, T. Z., \& Overgaard, M. (2004). Introspection and subliminal perception. Phenomenology and the Cognitive Sciences, 3(1), 1-23. doi: 10.1023/B:PHEN.0000041900.30172 .$e 8$

Reeves, A. (1982). Metacontrast U-shaped functions derive from two monotonic processes. Perception, 11(4), 415-426. doi: 10.1068/p110415

Samaha, J., \& Postle, B. R. (2015). The Speed of Alpha-Band Oscillations Predicts the Temporal Resolution of Visual Perception. Current Biology, 25(22), 2985-2990. doi: 10.1016/j.cub.2015.10.007

Schiller, P. H., \& Greenfield, A. (1969). Visual masking and the recovery phenomenon. Perception $\mathcal{E}$ Psychophysics, 6(3), 182-184. doi: 10.3758/BF03210090

Schwiedrzik, C. M., Singer, W., \& Melloni, L. (2009). Sensitivity and perceptual awareness increase with practice in metacontrast masking. Journal of Vision, 9(10), 18. doi: 10 $.1167 / 9.10 .18$

Slotnick, S. D., Klein, S. A., Carney, T., \& Sutter, E. E. (2001). Electrophysiological estimate of human cortical magnification. Clinical Neurophysiology, 112(7), 1349-1356. doi: 10.1016/S1388-2457(01)00561-2

Stewart, A. L., \& Purcell, D. G. (1974). Visual backward masking by a flash of light: A study of U-shaped detection functions. Journal of Experimental Psychology, 103(3), 553-566. doi: $10.1037 / \mathrm{h} 0037151$

Stigler, R. (1910). Chronophotische Studien über den Umgebungskontrast. Pflüger's Archiv für die Gesammte Physiologie des Menschen und der Tiere, 134(6-8), 365-435. doi: 10.1007/BF01680352 


\section{References}

Stojanoski, B., \& Niemeier, M. (2011). The timing of feature-based attentional effects during object perception. Neuropsychologia, 49(12), 3406-3418. doi: 10.1016/ j.neuropsychologia.2011.08.017

Stoper, A. E., \& Banffy, S. (1977). Relation of split apparent motion to metacontrast. Journal of Experimental Psychology: Human Perception and Performance, 3(2), 258-277. doi: 10.1037//0096-1523.3.2.258

Sturr, J. F., Frumkes, T. E., \& Veneruso, D. M. (1965). Spatial determinants of visual masking: Effects of mask size and retinal position. Psychonomic Science, 3(1-12), 327-328. doi: 10.3758/BF03343163

Tata, M. S. (2002). Attend to it now or lose it forever: Selective attention, metacontrast masking, and object substitution. Perception E Psychophysics, 64(7), 1028-1038. doi: 10.3758/ BF03194754

Tse, P. U., Martinez-Conde, S., Schlegel, A. A., \& Macknik, S. L. (2005). Visibility, visual awareness, and visual masking of simple unattended targets are confined to areas in the occipital cortex beyond human V1/V2. Proceedings of the National Academy of Sciences, 102(47), 17178-17183. doi: 10.1073/pnas.0508010102

Vaughan, H. G., \& Silverstein, L. (1968). Metacontrast and Evoked Potentials: A Reappraisal. Science, 160(3824), 207-208. doi: 10.1126/science.160.3824.207

Ventura, J. (1980). Foveal metacontrast: I. Criterion content and practice effects. Journal of Experimental Psychology: Human Perception and Performance, 6(3), 473-485. doi: 10.1037//0096-1523.6.3.473

Vorberg, D., Mattler, U., Heinecke, A., Schmidt, T., \& Schwarzbach, J. (2003). Different time courses for visual perception and action priming. Proceedings of the National Academy of Sciences, 100(10), 6275-6280. doi: 10.1073/pnas.0931489100

Weisstein, N. (1966). Backward masking and models of perceptual processing. Journal of Experimental Psychology, 72(2), 232-240. doi: 10.1037/h0023437

Weisstein, N. (1970). Neural Symbolic Activity: A Psychophysical Measure. Science, 168(3938), 1489-1491. doi: 10.1126/science.168.3938.1489

Weisstein, N. (1972). Metacontrast. In M. Alpern et al. (Eds.), Visual Psychophysics (Vol. 7/4, pp. 233-272). Berlin, Heidelberg: Springer Berlin Heidelberg. doi: 10.1007/978-3-642 -88658-4\textunderscore 10

Weisstein, N., \& Growney, R. L. (1969). Apparent movement and metacontrast: A note on Kahneman's formulation. Perception $\mathcal{E}$ Psychophysics, 5(6), 321-328. doi: 10.3758/ BF03210651

Weisstein, N., Ozog, G., \& Szoc, R. (1975). A comparison and elaboration of two models of metacontrast. Psychological Review, 82(5), 325-343. doi: 10.1037/0033-295X.82.5.325 
Wutz, A., Weisz, N., Braun, C., \& Melcher, D. (2014). Temporal windows in visual processing: p̈restimulus brain stateänd p̈oststimulus phase reset⿱segregate visual transients on different temporal scales. The Journal of neuroscience : the official journal of the Society for Neuroscience, 34(4), 1554-1565. doi: 10.1523/JNEUROSCI.3187-13.2014 



\section{List of Figures}

1.1 Meta- and paracontrast masking stimuli. . . . . . . . . . . . 2

2.1 Stimuli and trial course. . . . . . . . . . . . . . . . 10

3.1 Experiment 1.1 and 1.3. Discrimination Sensitivity $d^{\prime} \ldots \ldots \ldots \ldots$. . . . . . 17

3.2 Experiment 1.1 and 1.3. Mask Bias $C_{M} \ldots \ldots \ldots \ldots \ldots$

3.3 Experiment 1.1. Factor analysis - biplot with factor loadings and factor scores. 19

3.4 Experiment 1.1. Correlation of Mask Bias $C_{M}$ with Factor A and B scores. . . . 20

3.5 Experiment 1.2. Discrimination Sensitivity $d^{\prime} \ldots \ldots \ldots \ldots . \ldots \ldots$

3.6 Experiment 1.2. Individual Discrimination Sensitivity $d^{\prime}$ functions. . . . . . . 22

3.7 Experiment 1.2. Correlation of Discrimination Sensitivity $d^{\prime}$ with Factor A and B scores. . . . . . . . . . . . . . . . . . . . . . 23

3.8 Experiment 1.2. Mask Bias $C_{M} \ldots \ldots \ldots \ldots \ldots \ldots \ldots$

3.9 Experiment 1.2. Individual Mask Bias $C_{M}$ functions. . . . . . . . . . . . 25

3.10 Experiment 1.2. Correlation of Mask Bias $C_{M}$ with Factor A and B scores. . . . 26

3.11 Experiment 1.3. Correlation of Discrimination Sensitivity $d^{\prime}$ and Mask Bias $C_{M}$ with Factor A and B scores. . . . . . . . . . . . . . . . . . . 27

4.1 Experiment 2.2 and 3.2. Trial course . . . . . . . . . . . . . . . 37

4.2 Experiment 2.1 and 2.3. Discrimination Sensitivity $d^{\prime} \ldots \ldots \ldots$. . . . . . . . 39

4.3 Experiment 2.1 and 2.3. Mask Bias $C_{M} \ldots \ldots \ldots \ldots$. . . . . . 40

4.4 Experiment 2.1. Factor analysis - biplot with factor loadings and factor scores. 41

4.5 Experiment 2.1. Correlation of Mask Bias $C_{M}$ with Factor A and B scores. . . . 42

4.6 Experiment 2.2. Discrimination Sensitivity $d^{\prime}$ and Mask Bias $C_{M} \ldots \ldots \ldots$. . . 43

4.7 Experiment 2.2. Individual Discrimination Sensitivity $d^{\prime}$ functions. . . . . . . . 44

4.8 Experiment 2.2. Correlation of Discrimination Sensitivity $d^{\prime}$ with Factor A and B scores. . . . . . . . . . . . . . . . . . . . 45

4.9 Experiment 2.2. Individual Mask Bias $C_{M}$ functions. . . . . . . . . . . 46

4.10 Experiment 2.2. Correlation of Mask Bias $C_{M}$ with Factor A and B scores. . . . 47

4.11 Experiment 2.3. Correlation of Discrimination Sensitivity $d^{\prime}$ and Mask Bias $C_{M}$ with Factor A and B scores. . . . . . . . . . . . . . . . . . . . 48 
5.1 Experiment 3.1 and 3.3. Discrimination Sensitivity $d^{\prime} \ldots \ldots \ldots \ldots$. . . . . 57

5.2 Experiment 3.1 and 3.3. Mask Bias $C_{M} \ldots \ldots \ldots \ldots \ldots$

5.3 Experiment 3.1. Factor analysis - biplot with factor loadings and factor scores. 59

5.4 Experiment 3.1. Correlation of Mask Bias $C_{M}$ with Factor A and B scores. . . . 60

5.5 Experiment 3.2. Discrimination Sensitivity $d^{\prime}$ and Mask Bias $C_{M} \ldots \ldots \ldots$. . . 61

5.6 Experiment 3.2. Individual Discrimination Sensitivity $d^{\prime}$ functions. . . . . . . . 62

5.7 Experiment 3.2. Correlation of Discrimination Sensitivity $d^{\prime}$ with Factor A and B scores. . . . . . . . . . . . . . . . . . . . 63

5.8 Experiment 3.2. Individual Mask Bias $C_{M}$ functions. . . . . . . . . . . . 64

5.9 Experiment 3.2. Correlation of Mask Bias $C_{M}$ with Factor A and B scores. . . . 65

6.1 Experiment 4.1.2. Percent correct performance for Type A and Type B groups. 76

6.2 Experiment 4.1 and 4.2. Discrimination Sensitivity $d^{\prime}$ and Response Biases $C_{R} /$

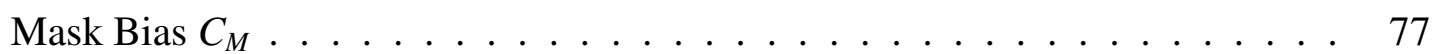

6.3 Experiment 4.1. Topographical plots and electrode sites. . . . . . . . . . . 79

6.4 Experiment 4.1. ERPs at PO7/PO8 of SOA $24 \mathrm{~ms} \ldots \ldots \ldots$

6.5 Experiment 4.1. ERPs at PO7/PO8 of SOA $72 \mathrm{~ms} \ldots \ldots \ldots$. . . . . . . 81

6.6 Experiment 4.1. ERPs at $\mathrm{O} 1 / \mathrm{O} 2$ of SOA $24 \mathrm{~ms} \ldots \ldots \ldots$. . . . . . . . . 82

6.7 Experiment 4.1. ERPs at $\mathrm{O} 1 / \mathrm{O} 2$ of SOA $72 \mathrm{~ms} \ldots \ldots \ldots 3$

7.1 Experiment 5.1. Discrimination Sensitivity $d^{\prime}$ and Mask Bias $C_{M} \ldots \ldots \ldots$. . . 91

7.2 Experiment 5.1. Factor analysis - biplot with factor loadings and factor scores. $\quad 92$

7.3 Experiment 5.1. Correlation of Mask Bias $C_{M}$ with Factor A and B scores. . . . 93

7.4 Experiment 5.2. Discrimination Sensitivity $d^{\prime}$ and Response Bias $C_{R}$. . . . . . 94

7.5 Experiment 5.2 Correlation of Discrimination Sensitivity $d^{\prime}$ and Response Bias $C_{R}$ with Factor A and B scores. . . . . . . . . . . . . . . . 96

7.6 Sustained-Transient-Dual-Channel Model. . . . . . . . . . . . . . . . . . . 98 


\section{List of Tables}

3.1 Experiment 1.1. Factor analysis - factor loadings. . . . . . . . . . . . 20

4.1 Experiment 2.1. Factor analysis - factor loadings. . . . . . . . . . . . . . 42

5.1 Experiment 3.1. Factor analysis - factor loadings. . . . . . . . . . . . 60

6.1 Experiment 4.1. Statistical results of the ANOVA at PO7/PO8. . . . . . . . . . 84

6.2 Experiment 4.1. Statistical results of the ANOVA at $\mathrm{O} 1 / \mathrm{O} 2 . \ldots \ldots 5$

7.1 Experiment 5.1. Factor analysis - factor loadings. . . . . . . . . . . 93 



\section{Abbreviations}

$\begin{array}{ll}\text { ANCOVA } & \text { analysis of covariance } \\ \text { ANOVA } & \text { analysis of variance } \\ \text { EEG } & \text { electroencephalogram } \\ \text { ERP } & \text { event-related potential } \\ \text { LGN } & \text { lateral geniculate nucleus } \\ \text { SOA } & \text { stimulus-onset-asynchrony } \\ \text { T/M ratio } & \text { target-to-mask energy ratio }\end{array}$





\section{Eigenständigkeitserklärung Declaration of Academic Honesty}

Hiermit erkläre ich, dass die vorliegende Arbeit ohne unzulässige Hilfe und ohne Benutzung anderer als der angegebenen Hilfsmittel angefertigt wurde und dass die aus fremden Quellen direkt oder indirekt übernommenen Gedanken in der Arbeit als solche kenntlich gemacht worden sind.

I hereby declare that this dissertation is my own work. Information derived from the published and unpublished work of others has been acknowledged in the text and a list of references is given in the bibliography.

Göttingen, 9. August 2016

Mareen Berndt 



\title{
Lebenslauf
}

\section{Curriculum Vitae}

\author{
Name: Mareen Berndt \\ Geburtstdatum: 28.10.1987 \\ Geburtsort: Görlitz, Deutschland
}

\author{
Name: Mareen Berndt \\ Date of Birth: 28.10 .1987 \\ Place of Birth: Goerlitz, Germany
}

\begin{abstract}
AUSBILDUNG
seit 03/2013 Wissenschaftliche Mitarbeiterin am Georg-Elias-Müller-Institut für Psychologie, Abteilung für Experimentelle Psychologie, GeorgAugust-Universität Göttingen (Deutschland), Promotionsstudiengang 10/2009-10/2012 Masterstudium Studiengang Integrative Neuroscience, Otto-vonGuericke-Universität Magdeburg (Deutschland), Abschluss: M.Sc. Neuroscience

10/2006 - 09/2009 Bachelorstudium Studiengang Biologie, Leibniz-Universität Hannover (Deutschland), Abschluss: B. Sc. Biologie

2006 Abitur, Augustum-Annen-Gymnasium, Görlitz (Deutschland)
\end{abstract}

\section{EDUCATION}

since 03/2013 PhD student at the Georg-Elias-Mueller-Institute for Psychology, Department for Experimental Psychology, Georg-August-University of Goettingen (Germany)

10/2009-10/2012 Master Studies Integrative Neuroscience, Otto-von-GuerickeUniversity of Magdeburg (Germany), Degree: M. Sc. Neuroscience

10/2006 - 09/2009 Bachelor Studies Biology, Leibniz-University of Hanover (Germany), Degree: B. Sc. Biology

2006 Abitur, Augustum-Annen-Gymnasium, Goerlitz (Germany) 
